\title{
Conditional Process Analysis in Two-Instance Repeated-Measures Designs
}

\author{
Dissertation
}

Presented in Partial Fulfillment of the Requirements for the Degree Doctor of Philosophy in the Graduate School of The Ohio State University

By

Amanda Kay Montoya, M.S., M.A.

Graduate Program in Department of Psychology

The Ohio State University

2018

Dissertation Committee:

Andrew F. Hayes, Advisor

Jolynn Pek

Paul de Boeck 
(c) Copyright by

Amanda Kay Montoya

2018 


\begin{abstract}
Conditional process models are commonly used in many areas of psychology research as well as research in other academic fields (e.g., marketing, communication, and education). Conditional process models combine mediation analysis and moderation analysis. Mediation analysis, sometimes called process analysis, investigates if an independent variable influences an outcome variable through a specific intermediary variable, sometimes called a mediator. Moderation analysis investigates if the relationship between two variables depends on another. Conditional process models are very popular because they allow us to better understand how the processes we are interested in might vary depending on characteristics of different individuals, situations, and other moderating variables. Methodological developments in conditional process analysis have primarily focused on the analysis of data collected using between-subjects experimental designs or cross-sectional designs. However, another very common design is the twoinstance repeated-measures design. A two-instance repeated-measures design is one where each subject is measured twice; once in each of two instances. In the analysis discussed in this dissertation, the factor that differentiates the two repeated measurements is the independent variable of interest. Research on how to statistically test mediation, moderation, and conditional process models in these designs has been minimal. Judd, Kenny, and McClelland (2001) introduced a piecewise method for testing for mediation, reminiscent of the Baron and Kenny causal steps approach for between-participant designs. Montoya and Hayes (2017) took this piecewise approach and translated it to a path-analytic approach, allowing for a quantification of the indirect effect, more sophisticated methods of inference, and the extension to multiple mediator models. Moderation analysis in these designs has been described by Judd, McClelland, and Smith (1996), Judd et al. (2001), and Montoya (in press). However, the generalization to conditional process analysis, or moderated mediation, remains unknown. Describing this approach is the purpose of this dissertation.
\end{abstract}


In this dissertation I propose a path analytic approach to assessing moderated mediation models for two-instance repeated-measures designs. In Chapter 1, I review the development of conditional process analysis in between-subjects designs. In Chapter 2, I review existing methods for mediation and moderation in two-instance repeated-measures designs. In Chapter 3, I provide a general framework for estimating conditional process models for two-instance repeated-measures designs with one moderator and one mediator. I describe how simplifications of this general model correspond to more commonly used conditional process models, such as first-stage conditional process models and second-stage conditional process models. In Chapter 4, I provide examples of the models described in Chapter 3 using data sets from a variety of areas of psychology. These examples show how to implement conditional process analysis and how to interpret the results of such an analysis. In Chapter 5, I discuss alternative methods for evaluating moderated mediation in two-instance repeated measures designs. I describe how the first-stage conditional process model in Chapter 3 is related to previous methods for testing mediation using a 2(within) X 2(between) design as described by MacKinnon (2008) and Valente and MacKinnon (2017). Two particularly popular alternative methodological approaches include multilevel models and structural equation modeling. I connect the regression based methods proposed in Chapter 3 to those described for 1-1-1 moderated mediation models. There is very little existing literature on assessing moderated mediation in repeated measures designs with structural equation models. Thus, I connect the proposed methods in this dissertation to existing approaches to mediation in repeated-measures designs with structural equation models: correlated residuals, latent-difference score models, latent-growth curve models, and cross-lag panel models. The final chapter provides a discussion of the general framework for approaching conditional process analysis in twoinstance repeated-measures designs, including future directions as well as limitations of the design and analytic framework presented. 
This dissertation is dedicated to the many trees killed in its creation. I hope your sacrifice was worth it. 


\section{Acknowledgments}

I'd like to acknowledge my adviser, Dr. Andrew F. Hayes, and my committee, Dr. Jolynn Pek and Dr. Paul De Boeck, for helping me through the dissertation process and guiding my research interests. Additional thanks to all of the researchers who provided data for this dissertation: Dr. Julie Lasselin and Ziv Bell. Thanks to my labmate Nick Rockwood for listening to me think about the ideas in this document and providing informal feedback, Dr. Trish Van Zandt for being a constant support throughout my journey as a graduate student. Thanks to all my fellow graduate students who shared in my stress, strive, and successes throughout the process of pursuing my degree. Thanks to my supportive family, Lori Montoya, Ric Montoya, and Jason Montoya for letting me fly the coop far from home to pursue my dream. A special thanks to my partner, Peter Kearns, who helped me maintain a life that was as balanced as one could hope for and helping me keep everything in perspective. Without all of these individuals this document, and thus my degree, would not exist. 


\section{Vita}

\begin{tabular}{|c|c|}
\hline May 8, 1991 & $\begin{array}{l}\text { Born } \\
\text { Seattle, WA }\end{array}$ \\
\hline 2011 & $\begin{array}{l}\text { A.A. Psychology, } \\
\text { North Seattle Community College }\end{array}$ \\
\hline 2013 & $\begin{array}{l}\text { B.S. Psychology } \\
\text { University of Washington }\end{array}$ \\
\hline 2016 & $\begin{array}{l}\text { M.A. Psychology } \\
\text { The Ohio State Univeristy }\end{array}$ \\
\hline 2016 & $\begin{array}{l}\text { M.S. Statistics } \\
\text { The Ohio State Univeristy }\end{array}$ \\
\hline 2014-present & $\begin{array}{l}\text { Graduate Fellow, } \\
\text { The Ohio State University }\end{array}$ \\
\hline
\end{tabular}

\section{Publications}

Hayes, A. F., \& Montoya, A. K. (2017). A tutorial on testing, visualizing, and probing an interaction involving a multicategorical variable in linear regression. Communication Methods and Measures, 11(1), 1-30.

Cheryan, S., Ziegler, S., \& Montoya, A. K., Jiang, L. (2017). Why have women entered some STEM fields more than others? Psychological Bulletin, 143(1), 1-35.

Montoya, A. K., \& Hayes, A. F. (2017). Two-condition within-participant statistical mediation analysis: A path-analytic framework. Psychological Methods, 22(1), 6-27.

Hayes, A. F., Montoya, A. K., \& Rockwood, N. J. (2017). The analysis of mechanisms and their contingencies: PROCESS versus structural equation modeling. Australasian Marketing Journal, 25(1), 76 - 81. 
Stojek, M. K., Montoya, A. K., Drescher, C., Newberry, A., Sultan, Z., Williams, C. F., Pollock, N. K., \& Davis, C. L. (2017). Fitness, sleep-disordered breathing, symptoms of depression, and cognition in inactive overweight children: Mediation models. Public Health Reports, 132(Supplement 2), 65S - 73S.

Dohle, S., \& Montoya, A. K. (2017). The dark side of fluency: Fluent names increase drug dosing. Journal of Experimental Psychology: Applied, 23(3), 231 -239.

Montoya, A. K. (2018). Probing moderation analysis in two-instance repeatedmeasures designs. Multivariate Behavior Research, 53(1), 140 - 141 [Abstract].

\section{Fields of Study}

Major Field: Department of Psychology 


\section{Table of Contents}

Page

Abstract .................................... ii

Dedication ................................. iv

Acknowledgments . . . . . . . . . . . . . . . . . . . . v v

Vita .................................... vi

List of Tables ........................ . . . . xi

List of Figures . . . . . . . . . . . . . . . . . . . xii

1. Mediation, Moderation, and Conditional Process Analysis in BetweenSubjects Designs . . . . . . . . . . . . . . . . 1

1.1 Introduction . . . . . . . . . . . . . . . . 1

1.2 Between-Subjects Designs . . . . . . . . . . . . . . . 7

1.3 Mediation . . . . . . . . . . . . . . . . . 10

1.3.1 A Path-Analytic Model . . . . . . . . . . . . . 10

1.3.2 Methods of Inference . . . . . . . . . . . . . . . . . . 12

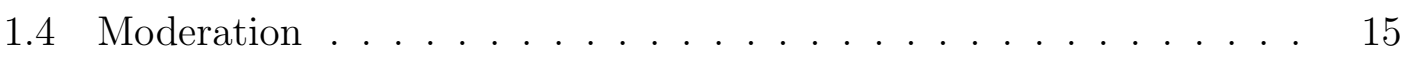

1.4.1 Testing an Interaction . . . . . . . . . . . . . . . 16

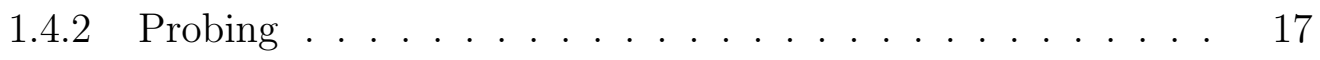

1.5 Conditional Process Analysis . . . . . . . . . . . . . . 24

1.5.1 A General Conditional Process Model . . . . . . . . . . . . 25

1.5.2 First-Stage Conditional Process Model . . . . . . . . . . . . 28

1.5.3 Second-Stage Conditional Process Model . . . . . . . . . . . 29

1.5.4 Methods of Inference . . . . . . . . . . . . . . . 30 
2. Mediation and Moderation in Two-Instance Repeated-Measures Designs

2.1 Two-Instance Repeated-Measures Designs . . . . . . . . . . . . . . 32

2.2 Mediation . . . . . . . . . . . . . . . . . . . . 34

2.2.1 A Path-Analytic Model . . . . . . . . . . . . 34

2.2.2 Estimation and Inference . . . . . . . . . . . . . . 41

2.3 Moderation ....................... 44

2.3.1 Testing an Interaction . . . . . . . . . . . . . . . 45

2.3.2 Estimation and Hypothesis Testing . . . . . . . . . . . . 48

2.3.3 Probing . . . . . . . . . . . . . . . . . . 48

2.4 Summary . . . . . . . . . . . . . . . . . . . 52

3. Conditional Process Analysis in Two-Instance Repeated-Measures Designs 53

3.1 A General Conditional Process Model . . . . . . . . . . . . . . 53

3.1.1 Defining Conditional Effects . . . . . . . . . . . 59

3.2 First Stage Conditional Process Models . . . . . . . . . . . . . . 62

3.3 Second Stage Conditional Process Models . . . . . . . . . . . . . . 65

3.4 Models where Instance is not a Moderator . . . . . . . . . . . . . . 67

3.5 Inference about Moderated Mediation . . . . . . . . . . . . . . . 70

3.5.1 Methods of Inference . . . . . . . . . . . . . . . 71

3.5.2 Probing Conditional Indirect Effects . . . . . . . . . . . . 73

3.6 Summary .......................... 74

4. Applications ......................... 77

4.1 Example 1: Lasselin et al. (2016) . . . . . . . . . . . . . . 78

4.2 Example 2: Bell, Shader, Webster-Stratton, Reid, Beauchaine (2017) 85

4.3 Example 3: Montoya, Master, Cheryan (2013) . . . . . . . . . . . . 92

5. Alternative Analytical Approaches . . . . . . . . . . . . . . . 100

5.1 Approaches to 2 (Within) X 2 (Between) Designs . . . . . . . . . . 101

5.1.1 Models for Pre- Post-Test Control Group Designs . . . . . . 101

5.1.2 Difference Score Model and Conditional Process Model . . . 109

5.1.3 ANCOVA Model and Conditional Process Models . . . . . . 110

5.2 Multilevel Conditional Process Models . . . . . . . . . . . . . . . . 113

5.2.1 1-1-1 Mediation . . . . . . . . . . . . . . . . 114

5.2.2 1-1-1 Conditional Process Models . . . . . . . . . . . . . . 118

5.2.3 Connection to Regression Based Method . . . . . . . . . . . 119

5.2.4 Advantages and Disadvantages of MLM approaches . . . . . 122

5.3 Structural Equation Approaches to Conditional Process Analysis . 124 
5.4 Summary . . . . . . . . . . . . . . . . . . . . 126

6. Discussion . . . . . . . . . . . . . . . . . . . . . . 128

6.1 Limitations . . . . . . . . . . . . . . . . . . . . . . . . . . 129

6.1 .1 Analytical Approach . . . . . . . . . . . . . . . . . 130

6.1 .2 Design . . . . . . . . . . . . . . . . . . . . . . 132

6.2 Future Directions . . . . . . . . . . . . . . . . . . . . . . . . 134

6.3 Summary . . . . . . . . . . . . . . . . 137

$\begin{array}{ll}\text { Appendices } & 139\end{array}$

A. Mplus code for Lasselin et al. (2016) analysis . . . . . . . . . . . . 139

B. Mplus code for Bell et al. (2017) analysis . . . . . . . . . . . . . . . . . 141

C. Mplus code for Montoya et al. (2013) analysis . . . . . . . . . . . . . 143

References . . . . . . . . . . . . . . . . . . . . . . . . . . 145 


\section{List of Tables}

Table

Page

3.1 The Index of Moderated Mediation and Conditional Indirect Effect with Second Order Asymptotic Variances for Three Moderated Mediation Models .................... . . . 75

4.1 Conditional Effects of Treatment on Pain with 95\% confidence intervals 84

4.2 Measured Variables by Time and Condition: Mean (Standard Deviation) 87

4.3 Conditional Effects of Time on PEP Reactivity with $95 \%$ confidence intervals . . . . . . . . . . . . . . . . . . . 90 


\section{List of Figures}

Figure $\quad$ Page

1.1 Path Diagram for Mediation Model for Between Subjects Design . . . 12

1.2 Path Diagram for Moderation Model for Between Subjects Design . . 18

1.3 Graph of relationship between hours of after-school experience and science performance moderated by age. . . . . . . . . . . . . . 19

1.4 Path Diagram for Conditional Process Model for Between Subjects Design . . . . . . . . . . . . . . . . . . 27

2.1 Path Diagram for Mediation Model for Two-Instance Repeated-Measures Design . . . . . . . . . . . . . . . . . . . . 40

4.1 Plot of interaction between time and inflammation on psychological inflexibility. . . . . . . . . . . . . . . . . . 81

4.2 Diagram for Lasselin et al. (2016) First-Stage Conditional Process Model. . . . . . . . . . . . . . . . . . . . . . . . . 83

4.3 Diagram for Bell et al. (2018) Second-Stage Conditional Process Model. 90

4.4 Diagram for Montoya et al. (2013) Mediation Model. . . . . . . . . 95

4.5 Diagram for Montoya et al. (2013) Conditional Process Model. . . . . 98 


\section{Chapter 1: Mediation, Moderation, and Conditional Process Analysis in Between-Subjects Designs}

\subsection{Introduction}

Mediation, moderation, and conditional process analysis addresses common questions in psychological research. Mediation investigates if an independent variable $(X)$ indirectly affects an outcome $(Y)$ through a mediator variable $(M$; also known as an "intermediary variable" or "intervening variable"). Mediation hypotheses address questions of why or how some effect occurs. Moderation helps us understand if the relationship between an independent variable $(X)$ and a dependent variable $(Y)$ depends on the level of a moderator $(W)$. Moderation analysis addresses questions of when or for whom certain effects occur. Conditional process analysis combines mediation and moderation, allowing us to examine if the effect of an independent variable $(X)$ on a dependent variable $(Y)$ through a mediator $(M)$ depends on a moderator $(W)$. This furthers our understanding of whether the indirect effect depends on the moderator or if the indirect effect is relatively consistent across the range of the moderator.

The idea of conditional process analysis, or moderated mediation, has been around since the 1980s (e.g., Baron \& Kenny, 1986; James \& Brett, 1984). It was not until the early 21st century that progress began in statistically testing hypotheses of 
moderated mediation. It is no coincidence that this was the same time that mediation analysis was moving away from the piecewise logic of Baron and Kenny. Muller, Judd, and Yzerbyt (2005) outlined a very general path-analytic model for moderated mediation, allowing for the path from $X$ to $M$, the path from $M$ to $Y$, and the path directly from $X$ to $Y$ to be moderated by the same moderator (See Figure 1.4). Using a similar piecewise logic to Baron and Kenny, Muller et al. (2005) suggested that different patterns of significance of sets of regression coefficients would suggest whether mediation is moderated. Edwards and Lambert (2007) proposed a variety of simpler moderated mediation models and provided instructions for how to estimate these models using path analysis and how to interpret the coefficients that were estimated. Preacher, Rucker, and Hayes (2007) described how to estimate a variety of moderated mediation models and how to use bootstrapping and normal-theory standard errors to conduct inference on conditional indirect effects. These two methods can be used to test if the indirect effect is significantly different from zero at a specific value of the moderator.

The early proposed methods and tests for moderated mediation allow for estimation of a moderated mediation model, but they did not have a direct test to answer the question "Is mediation moderated?" A method proposed by Fairchild and MacKinnon (2009) provided a test of moderated mediation if the moderator was dichotomous. Hayes (2015) introduced the index of moderated mediation which provided an answer to the question of moderated mediation for continuous and dichotomous moderators alike. This index quantifies the degree to which the indirect effect depends on a moderator. A test on this index, typically conducting using bootstrap confidence intervals, allows for direct inference on whether this parameter is different from zero. 
If the index is significantly different from zero, then there is evidence of moderated mediation. Extensions of the index of moderated mediation can be used in moderated mediation models where there is more than one moderator or where one moderator is moderating more than one path in the mediation pathway (Hayes, 2018b).

Developments in conditional process analysis have been primarily focused on analysis with data collected using between-subjects experimental designs or cross-sectional designs. However, another very common design in psychology is the two-instance repeated-measures design. A two-instance repeated-measures design is a design where each subject is measured on the outcome twice, once in each of two instances, and the factor which differentiates the instances (e.g., happy story vs. sad story, pre vs. post treatment) is the independent variable of interest. Research on how to statistically test mediation, moderation, and conditional process models in these designs is minimal. Judd et al. (2001) introduced a piecewise method for testing for mediation, reminiscent of the Baron and Kenny causal steps approach. Montoya and Hayes (2017) took this piecewise approach and translated it to a path-analytic approach, allowing for a quantification of the indirect effect, more sophisticated methods of inference, and the expansion to more complex models. Moderation for two-instance repeated-measures designs had been described by Judd et al. (1996), Judd et al. (2001), and Montoya (in press). Montoya and Hayes (2017) showed extensions to multiple mediator models, including mediators in parallel and in serial. However, the generalization to conditional process analysis, or moderated mediation, has not yet been described.

In this dissertation I define a path analytic modeling approach that can be used to estimate and conduct inference on all the parameters of interest in a conditional 
process model for a two-instance repeated-measures design. Additionally, I provide some examples of estimating these models with psychological data. I also discuss alternative methods to the proposed approach.

Expanding conditional process analysis to the two-instance repeated-measures design has the potential to impact the field of psychology in a variety of ways. One issue in psychology research which impedes development is "methodological silos." Areas of psychology are often very homogeneous in their study design and measures of constructs such that specific methodologies become tied to specific areas of inquiry. This can be incredibly limiting for theory development. Statistical methods are tied to study design. If a research area tends to use a design for which a statistical method is not developed for answering specific types of questions, then it is unlikely for such questions to be answered in that area. For example, if a hypothetical group of researchers was only familiar with single factor designs and were not familiar with 2-Way ANOVA or models which include multiple factors, this area would be unlikely to investigate questions of interaction or moderation, since this involves two factors where the effect of one factor depends on the level of the other factor. Some areas of psychology which use two-instance repeated measures designs may be unfamiliar with tests of mediation or moderated mediation, as there is very little research on this type of analysis with their design of choice. By expanding moderated mediation to two-instance repeated measures designs, areas that typically use this design may develop more nuanced theories surrounding mechanisms and the moderation of those mechanisms. Before the introduction of the methods presented in this dissertation, researchers in these areas may not have considered moderation of mechanisms because 
there was no way to test these types of theories. By introducing this new possibility, specific areas of psychology can expand their theoretical models to considering mechanisms and what might affect these mechanisms.

A second way that this dissertation will impact the field of psychology is that it will address inappropriate statistical approaches to answering questions of moderated mediation in two-instance repeated measures designs. As an example, Winterich, Mittal, and Ross (2009) examined how group (in-group vs. out-group) influenced donating amount by including the recipient as part of one's self, and how this effect is moderated by gender identity and moral identity. Overall, they showed support for their model by using a series of regression analyses and examining the statistical significance of specific paths. However, this method does not allow the researcher to directly test if the mediation is moderated using a single test. Additionally, without a clearly identified path model, the researchers could not estimate indirect effects for specific combinations of the moderator values. The methods proposed in this dissertation will allow future researchers to more directly test their questions of moderated mediation, and estimate effects most directly of interest when investigating moderated mediation hypotheses.

One of the goals of introducing conditional process analysis to two-instance repeatedmeasures designs is to potentially expand the design possibilities in areas of research that depend on conditional process analysis. It could be that researchers already very familiar with conditional process analysis could benefit from using a withinparticipant design. One benefit of this design is that you can circumvent betweenparticipant variation in the data when estimating effects. This means that if you are interested in purely within-participant change or difference, then using these types 
of designs may give more accurate and more powerful tests related to your question of interest. If, however, the theoretical question of interest involved moderated mediation, it would not be unreasonable to choose a design for which this type of analysis is more developed. By expanding conditional process analysis to two-instance repeated-measures designs, this dissertation opens the door of design possibilities to researchers, providing the opportunity to use statistical methods with greater statistical power and more precise parameter estimates.

Two-instance repeated-measures designs provide a nice introduction to repeatedmeasures designs. Researchers familiar with mediation, moderation, and conditional process analysis in between-subjects designs will see familiar concepts in the methods described in this dissertation. From there it is not a far jump to ask "What if we measure people over three time points instead of two?" This would bring the researcher to the existing literature on longitudinal mediation analysis (Cheong, MacKinnon, \& Khoo, 2003; Cole \& Maxwell, 2003; Roth \& MacKinnon, 2012; von Soest \& Hagtvet, 2011). Alternatively, the researcher might ask "What if I measure people multiple times in each condition?" This would lead the researcher to the existing literature on multilevel mediation analysis (Krull \& MacKinnon, 2001; Kenny, Korchmaros, \& Bolger, 2003; Bauer \& Curran, 2005; Bauer, Preacher, \& Gil, 2006; Selig \& Preacher, 2009). The two-instance repeated-measures design provides a bridge between between-subjects designs and more complicated repeated-measures designs which require more advanced estimation strategies than ordinary least squares regression. This dissertation begins to bridge the approaches to mediation, moderation, and moderated mediation in between-subjects designs and in multilevel designs. 
Chapters $1-4$ introduce conditional process analysis in the two-instance repeatedmeasures design to an audience that may only be familiar with these analyses in a between-subjects design. Chapter 5 discusses the connection between the methods described in this dissertation for two-instance repeated-measures design and more complex models which are best suited for extensions of the two-instance repeatedmeasures design, thus bridging the gap among these existing literatures.

I begin with a review of mediation, moderation, and conditional process models in between-subjects designs. This framework provides the foundation for developing similar models in two-instance repeated-measures designs.

\subsection{Between-Subjects Designs}

I use the term between-subjects designs to describe two very popular designs in psychological research: (1) the between-subjects experimental design and (2) crosssectional designs. In a between-subjects experimental design, participants are randomly assigned to a condition $(X)$ and then measured on the mediator $(M)$ and the outcome variable $(Y)$. A moderator $(W)$ is ideally measured before the participant undergoes the manipulation to ensure the moderator is not affected by the manipulation. As an example of a between-subjects experimental design, imagine we are studying how making decisions under uncertainty impacts optimism about future decisions through confidence in decision making (e.g., Tong, Feiler, \& Ivantsova, 2017). We could randomly assign individuals to make a choice under certainty (where they have enough information to know what the correct decision is) or under uncertainty (they have some information which points to the correct decision, but not enough to be completely certain). In this design each person is randomly assigned to one of 
the two conditions $(X)$ and then makes a decision. Next, regardless of their decision, participants are told that they were correct in the first decision. Next we measure the participant's confidence in their decision making abilities $(M)$. We might expect those who made the decision under uncertainty to have higher confidence than those who decided under certainty. Finally, participants are told that they will make a second decision where the information is not completely available (uncertainty). Before making the decision, participants rate how optimistic they are they will make the correct decision $(Y)$. The example suggests how one might use a between-subjects experimental design to assess if decision making uncertainty impacts optimism through confidence.

In cross-sectional designs there is no experimental manipulation, but all variables are measured including the independent variable of interest $(X)$, the mediator $(M)$, the moderator $(W)$, and the outcome variable $(Y)$. A cross-sectional design could also be used to examine the relationship between decision making uncertainty and optimism through confidence. As an example, we might ask participants to think of a recent decision they made when they felt they were correct in their decision. Next we could ask the participants to rate the level of uncertainty when they made that decision. This measure would be a measure of $X$, how much they felt they had complete information in making the decision. Note that $X$ is no longer a dichotomous experimental manipulation, but rather a continuous observed variable. Next the study might operate very similarly to the between-subjects experimental design just described, where participants rate their confidence in decision making $(M)$, are told they will make a decision under uncertainty, and then rate their optimism that they would make the correct decision $(Y)$. Both between-subjects experimental designs and 
cross-sectional designs can be used to assess similar questions; however experimental designs have the added benefit of clearer understanding of causality.

The data for these two designs are treated equivalently when conducting mediation, moderation, and conditional process analysis. This is because the structure of the data is the same, where each individual is measured on 3 variables (for a mediation or moderation analysis) or four variables (for a conditional process analysis) plus any covariates. A primary difference between these two designs is the strength of causal inference. When $X$ is randomly assigned, the estimate of the effect of $X$ on $Y$ is an estimate of a causal effect. In the first decision making example, individuals are randomly assigned to condition, so the estimated effect of decision-making uncertainty on both confidence and optimism are causal in nature. But when $X$ is not randomly assigned, there is no way to know which variable causes which. For example, in the cross-sectional design described above, we would not know if people's optimism influences their uncertainty in decision making (where $Y$ is causing $X$ ) or if decision uncertainty is causing optimism (where $X$ is causing $Y$ ). Because mediation analysis assumes the causal order of the variables is correctly specified, experimental manipulation of $X$ (when possible and ethical) is highly recommended. This aids the interpretation of at least some of the mediation paths as causal in nature. However, even when $X$ is randomly assigned, not all paths in the mediation model can be assumed to be causal in nature. Part of the mediation model assumes that $M$ causes $Y$, but without random assignment on $M$ there is nothing in the design that guarantees this is the case. Researchers often must rely on theory or secondary experiments to support this assumption in a mediation model. 


\subsection{Mediation}

Mediation is when an independent variable $(X)$ has an indirect effect on an outcome variable $(Y)$ through an intermediary variable or mediator $(M)$. An indirect effect is one where the independent variable affects the mediator, and that subsequent change in the mediator affects change in the outcome. Mediation analysis is a statistical procedure to investigate whether such effects occur. Modern mediation analysis has allowed researchers from a variety of areas in psychology to examine and test questions of process: What is the process through which one variable exerts an effect on another? Mediation analysis is very popular. Hayes and Scharkow (2013) found that $15.6 \%$ of papers in Psychological Science had mediation analyses. Fritz and MacKinnon (2007) looked at papers from the Journal of Consulting and Clinical Psychology and the Journal of Applied Psychology over 3 years and found 166 articles contained mediation analysis. Frazier, Tiz, and Barron (2004) found that $19 \%$ of articles in the Journal of Counseling Psychology contained mediation analysis. Mediation analysis has become core to the field of psychology. More complete introductions to mediation analysis can be found in Preacher et al. (2007), Hayes (2018a), MacKinnon (2008), and others.

\subsubsection{A Path-Analytic Model}

Modern approaches to mediation analysis use a path-analytic framework to estimate the indirect effect and conduct inference based on the estimate of the indirect effect. Throughout this dissertation I assume that $Y$ and $M$ are both quantitative continuous variables (i.e., not dichotomous or polytomous). Three regression equations are used to define a mediation model: 


$$
\begin{aligned}
Y_{i} & =c_{0}+c X_{i}+e_{Y_{i}} \\
M_{i} & =a_{0}+a X_{i}+e_{M_{i}} \\
Y_{i} & =c_{0}^{\prime}+c^{\prime} X_{i}+b M_{i}+e_{Y_{i}^{\prime}}
\end{aligned}
$$

In these equations, $i$ denotes individual, and $X_{i}, M_{i}$, and $Y_{i}$ note individual $i$ 's observation on the independent variable, the mediator, and the outcome, respectively. The intercepts for each equation are represented by $c_{0}, a_{0}$, and $c_{0}^{\prime}$. The total effect, is denoted $c$, which is the regression coefficient for $X$ when predicting $Y$ and the mediator is not included in the model. The coefficient for $X$ when predicting $M$ is denoted $a$. The regression coefficient for $X$ in predicting $Y$ controlling for $M$ is $c^{\prime}$ and is the direct effect. The coefficient for $M$ in predicting $Y$ is denoted $b$. Throughout this dissertation I use "hat" notation to denote estimates of population parameters. For example $\hat{b}_{1}$ is the estimate of the population parameter $b_{1}$.

Figure 1.1 shows how by tracing the paths from $X$ to $Y$ through $M$, the $a$-path and the $b$-path define the indirect effect of $X$ on $Y$ through $M$. Path analysis algebra shows that the indirect effect is the product of the estimates of $a$ and $b$. Manipulating the regression equations above shows how the total effect can be described as the sum of the indirect effect, $a b$, and the direct effect $c^{\prime}$. Plugging in Equation 1.2 as $M$ in Equation 1.3 and grouping terms, the coefficient for $X$ is the sum of $a b$ and $c^{\prime}$.

$$
\begin{aligned}
Y_{i} & =c_{0}^{\prime}+c^{\prime} X_{i}+b\left(a_{0}+a X_{i}+e_{M_{i}}\right)+e_{Y_{i}^{\prime}} \\
& =c_{0}^{\prime}+c^{\prime} X_{i}+b a_{0}+a b X_{i}+b e_{M_{i}}+e_{Y_{i}^{\prime}} \\
& =\left(c_{0}^{\prime}+b a_{0}\right)+\left(c^{\prime}+a b\right) X_{i}+\left(b e_{M_{i}}+e_{Y_{i}^{\prime}}\right)
\end{aligned}
$$


This shows how Equations 1.2 and 1.3 can be combined to be equivalent to Equation 1.6, where $c_{0}=c_{0}^{\prime}+b a_{0}, c=c^{\prime}+a b$, and $e_{Y_{i}}=b e_{M_{i}}+e_{Y_{i}^{\prime}}$. So, the total effect is the sum of the direct effect and indirect effect. Because the indirect effect quantifies the degree to which $X$ affects $Y$ through $M$, modern approaches to mediation analysis estimate the indirect effect and conduct inference based on that estimate. It is worth noting that this decomposition method only works with ordinary least squares regression and not for other methods like logistic regression (but see Breen, Karlson, \& Holm, 2013).
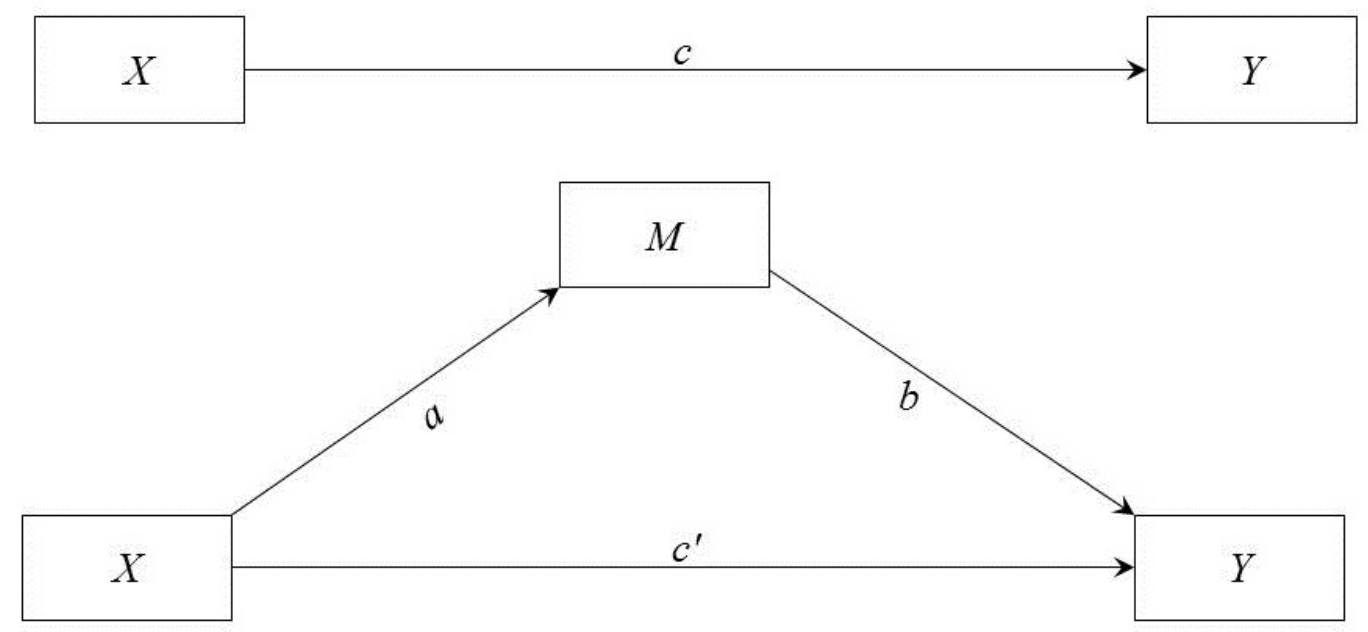

Figure 1.1: Path Diagram for Mediation Model for Between Subjects Design

\subsubsection{Methods of Inference}

The indirect effect, direct effect, and total effect can be estimated using ordinary least squares. Inference for the direct and total effect, such as hypothesis testing and 
creating confidence intervals, can be conducted using the standard error estimates from OLS, typically used for linear regression. The indirect effect is unique because it is the product of two regression coefficients, and so other hypothesis testing techniques are needed.

The causal steps method was the earliest method for testing mediation hypotheses to gain popularity (Baron \& Kenny, 1986). To claim statistical mediation, Baron and Kenny (1986) required rejection of the null hypothesis for three hypothesis tests: (1) there is an effect of $X$ on $Y$ (i.e., $c$ from Equation 1.1), (2) there is an effect of $X$ on $M$ (i.e., $a$ from Equation 1.2), and (3) there is an effect of $M$ on $Y$ controlling from $X$ (i.e., $b$ from Equation 1.3). If each of these tests is statistically significant, we claim that there is mediation. This method requires first testing that the total effect is significant before testing the individual paths of the mediation model. Though many methodologists have spoken out against testing for a significant total effect to predicate a test of mediation (Collins, Graham, \& Flaherty, 2010; Kenny, Kashy, \& Bolger, 1998; MacKinnon, 2008; Hayes, 2018a), this practice is still relatively common. One issue with requiring there to be a significant total effect to claim mediation is that when the direct effect is zero (i.e., the indirect effect is equal to the total effect) we can have more power to detect the indirect effect than the total effect (Kenny \& Judd, 2014). At the population level, it possible that the effect of $X$ on $Y$ through $M$ (the indirect effect) is positive and the effect of $X$ on $Y$ not through $M$ (the direct effect) is negative and of an equal value such that the total effect is exactly zero. In this case, there is no total effect of $X$ on $Y$ even though there is an indirect effect of $X$ on $Y$. The opposite is also possible where the indirect effect can be negative and the direct effect is positive; such an effect is termed "inconsistent 
mediation" (Davis, 1985). At the sample level effects which are not exactly equal can still balance out, such that inference about the indirect effect should not be conditional on a significant total effect. It is useful to think about mediation as a series of effects, where $X$ affects $M$ and $M$ then affects $Y$; we do not need to require a significant total effect in order to test a mediation hypothesis.

A final test from the Baron and Kenny (1986) method provides evidence of what Baron and Kenny (1986) called "full" or "partial" mediation. They claim that if the direct effect is significantly different from zero, there is still a remaining relationship between $X$ and $Y$ and so the mediator only partially explains the relationship between $X$ and $Y$. However, if the direct effect is not significantly different from zero, then this is called "complete" mediation, meaning that the mediator completely explains the relationship between $X$ and $Y$. There are many flaws in the ideas of partial and complete mediation, and so experts in the field recommend abandoning this practice as well (see e.g., Hayes, 2009; Rucker, Preacher, \& Tormala, 2011).

Another early method for conducting inference on the indirect effect is called the Sobel test. Sobel (1982) used the Delta Method to show that the asymptotic distribution of the indirect effect is normal and derived the asymptotic standard error of the indirect effect. To conduct a Sobel test, the ratio of the estimate of the indirect effect to it's asymptotic standard error is assumed to have a standard normal distribution under the null hypothesis that the indirect effect is zero. Based on the observed test statistic and the standard normal distribution, a $p$-value can be calculated and used for hypothesis testing. Alternatively confidence intervals can be calculated using the standard error and critical $z$-values. However it has been shown 
that the finite sampling distribution of the indirect effect is not always normally distributed (Stone \& Sobel, 1990).

Alternative methods have been developed which respect the non-normality of the sampling distribution of the indirect effect by either using the distribution of the product of two normally distributed variables (MacKinnon, Lockwood, Hoffman, West, \& Sheets, 2002), simulating the distribution of the product of two normally distributed variables (Selig \& Preacher, 2009), or by empirically estimating the distribution of the indirect effect using bootstrapping (Shrout \& Bolger, 2002; Preacher \& Hayes, 2004). These methods have been shown to perform better than the methods that assume the distribution of the indirect effect is normal (Williams \& MacKinnon, 2008; MacKinnon, Lockwood, \& Williams, 2004; Hayes \& Scharkow, 2013; Biesanz, Falk, \& Savalei, 2010). I will describe these methods more in depth in Chapter 2.

\subsection{Moderation}

In moderation analysis for a between-subjects design, all variables involved are measured once for each participant. Each individual has a measurement on the focal predictor $(X)$, moderator $(W)$, and outcome variable $(Y)$. In this section, I provide a short review of the principles of testing and probing interactions in betweenparticipant designs. More extensive introductions to moderation analysis in betweenparticipant designs can be found in Hayes (2018a), Jaccard and Turrisi (2003), Cohen, Cohen, West, and Aiken (2003), and many others.

In a standard multiple linear regression, the relationship between focal predictor $(X)$ and outcome $(Y)$ is constant with respect to the second independent variable $(W)$. 


$$
Y_{i}=b_{0}^{*}+b_{1}^{*} X_{i}+b_{2}^{*} W_{i}+e_{Y_{i}^{*}}
$$

In this model, the outcome variable for participant $i, Y_{i}$, is a linear function of both participant $i$ 's responses on focal predictor $X_{i}$ and their response on $W_{i}$. The error in estimating person $i$ 's response on $Y_{i}$ with this combination of $X_{i}$ and $W_{i}$ is represented by $e_{Y_{i}^{*}}$. The coefficient $b_{0}^{*}$ corresponds to the intercept which is the predicted value of $Y$ when $X$ and $W$ are both zero. The relationship between $X$ and $Y$ controlling (or holding constant) $W$ is $b_{1}^{*}$. The coefficient $b_{2}^{*}$ can be interpreted as the relationship between $W$ and $Y$ holding $X$ constant. I use the superscript $*$ on the coefficients in this equation to make clear that they are different from those estimated later denoted without the $*$ superscript.

\subsubsection{Testing an Interaction}

We can test for moderation by allowing the relationship between $X$ and $Y$ to be a function of $W, f\left(W_{i}\right)$.

$$
Y_{i}=b_{0}+f\left(W_{i}\right) X_{i}+b_{2} W_{i}+e_{Y_{i}}
$$

In this model, the outcome variable for participant $i, Y_{i}$, is a function of both participant $i$ 's responses on focal predictor, $X_{i}$, and their response on the moderator, $W_{i}$. The error in estimating person $i$ 's response on $Y_{i}$ with this combination of $X_{i}$ and $W_{i}$ is represented by $e_{Y_{i}}$. The coefficients $b_{0}$ corresponds to the intercept (predicted value of $Y$ when $X$ and $W$ are both zero). The relationship between $X$ and $Y$ is $f\left(W_{i}\right)$. Regardless of the choice of $f\left(W_{i}\right)$, the coefficient $b_{2}$ can be interpreted as the 
relationship between $W$ and $Y$ when $X$ is zero. In order to estimate this model, a form for $f\left(W_{i}\right)$ must be chosen. A linear function is typically used:

$$
f\left(W_{i}\right)=b_{1}+b_{3} W_{i}
$$

This analysis is described as linear moderation analysis, where the "linear" is describing the choice of function, $f\left(W_{i}\right)$. When a linear function is used, the product between $X$ and $W$ is added to Equation 1.8:

$$
\begin{aligned}
Y_{i} & =b_{0}+\left(b_{1}+b_{3} W_{i}\right) X_{i}+b_{2} W_{i}+e_{Y_{i}} \\
& =b_{0}+b_{1} X_{i}+b_{2} W_{i}+b_{3} X_{i} W_{i}+e_{Y_{i}}
\end{aligned}
$$

This equation can be estimated using any multiple linear regression program. When the parameter $b_{3}$ is zero, the relationship between $X$ and $Y$ does not depend on $W$ (i.e., $b_{1}+0 W=b_{1}$ ). To test a moderation or interaction hypothesis, an inferential test on the estimate of $b_{3}$ gives an indication of whether the relationship between $X$ and $Y$ depends linearly on $W$. A path diagram of Equation 1.11 is included in Figure 1.2 .

\subsubsection{Probing}

With evidence of moderation or interaction, our focus shifts toward the pattern of effects. There are many ways that the effect of one variable on another can depend on a third, and probing the interaction helps describe that pattern. Probing analyses are typically most informative when a test of interaction is significant, though there 


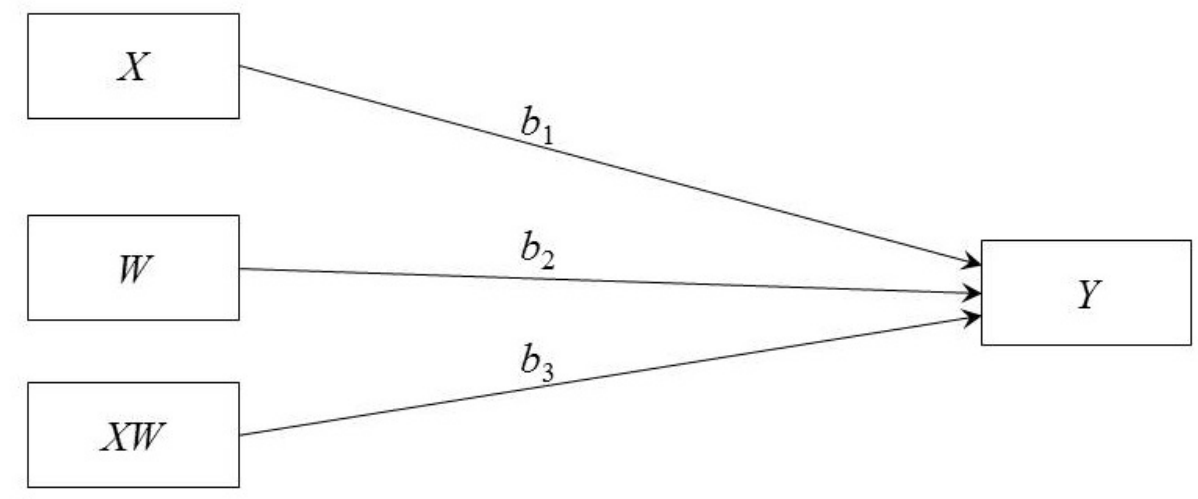

Figure 1.2: Path Diagram for Moderation Model for Between Subjects Design

may be justifiable reasons why we might want to probe an interaction that is not significant. Researchers who probe non-significant interactions may find themselves grappling with difficult to explain results due to the dichotomous decision making procedures of null hypothesis testing. It is important to remember that a difference in significance does not imply significantly different: One conditional effect may be significantly different from zero and a second may not. This does not mean that these two conditional effects are significantly different from each other.

Figure 1.3 shows an example of moderation, where $X$ is a continuous variable representing hours of experience, ranging from 0 to 10 in the observed data, $W$ is a continuous variable representing age (coded based on years of age), and $Y$ represents performance on a standardized science test (with scores ranging from 0 to 100). The graph in Figure 1.3 corresponds to the equation:

$$
\hat{Y}_{i}=40-2 X_{i}+1 W_{i}+0.5 X_{i} W_{i}
$$




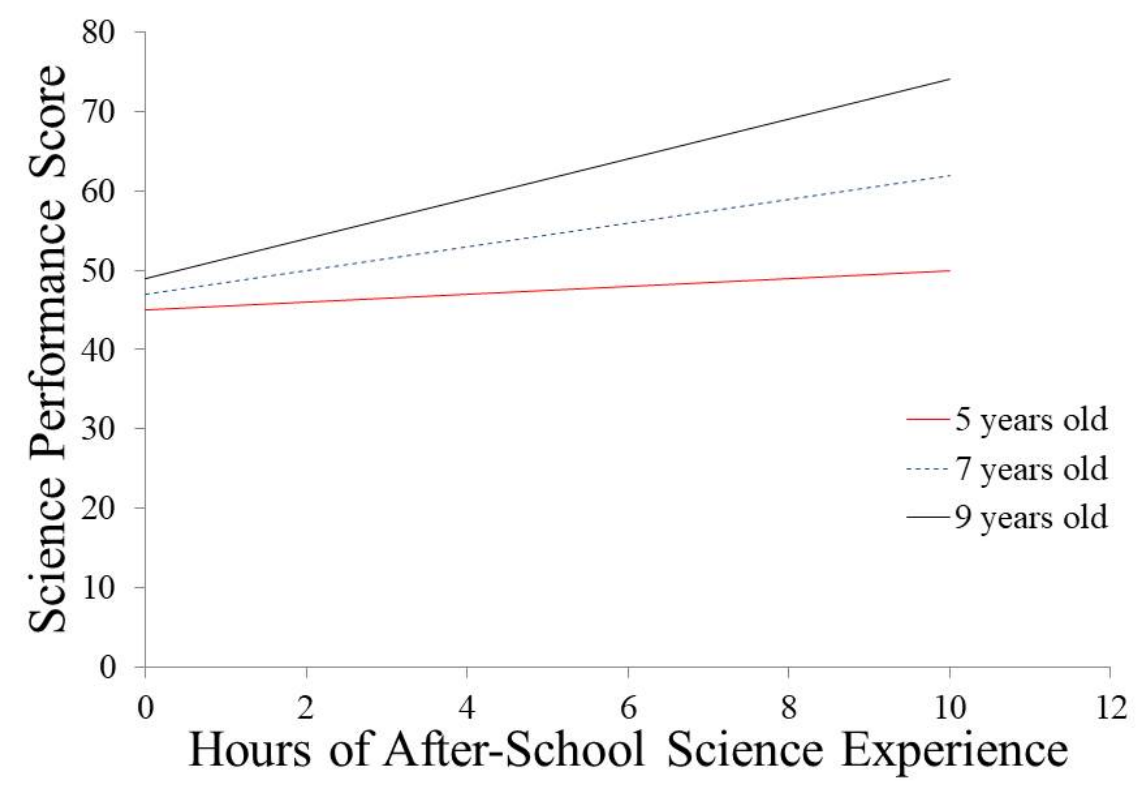

Figure 1.3: Graph of relationship between hours of after-school experience and science performance moderated by age.

Looking at Figure 1.3 and Equation 1.12, the slope of the line for children who are 5 years old is 0.5 . When children are 7 , the slope is 1.5 . The difference between these two slopes is 1 , which is twice of $\hat{b}_{3}=0.5$ because 5 year olds and 7 year olds are two units different from each other on $W$. A hypothesis test on the coefficient $\hat{b}_{3}$ is a test of whether the slopes of the two lines are different from one another. Given these estimates and some corresponding hypothesis tests, assume we conclude the relationship between hours of after-school experience and science performance is different for children of different ages (i.e., $b_{3} \neq 0$ ). Graphically this means that the slope of the line for 5 year olds is different than the slope of the line for 7 year olds is different from the slope of the line for 9 year olds. Figure 1.3 shows that older 
children seem to benefit more from increases in after-school science experience. But do children of all ages benefit from experience? Are the slopes of each of these lines different from zero? These questions can be answered with probing analysis.

The function $b_{1}+b_{3} W_{i}$, which I denote $\theta_{X \rightarrow Y}(W)$, is the conditional effect of $X$ on $Y$ as a function of $W$ (e.g., the conditional effect of after-school science experience on science performance as a function of age). While probing an interaction, $\theta_{X \rightarrow Y}(W)$ is estimated and inference is conducted at different values of $W$. We can now examine the relationship between after-school experience and science performance for 5 year olds, 7 year old, and 9 year olds separately. Using Equation 1.12, the conditional effect of $X$ on $Y$ can be calculated for children of any age, but are these slopes significantly different from zero? We can answer this through one of two primary methods of probing an interaction: the pick-a-point approach and the Johnson-Neyman procedure.

\section{Pick-a-point}

The pick-a-point approach (a.k.a., simple-slopes analysis or "spotlight analysis", Spiller, Fitzimons, Lynch Jr., \& McClelland, 2013) is a method for probing an interaction by selecting a specific value of the moderator $W$ and then estimating and conducting inference on the conditional effect of the focal predictor $X$ on the outcome $Y$ at that specific value of the moderator $W$. This helps to answer the question, "Is there an effect of $X$ on $Y$ at $w ? "$ where $w$ is a fixed value of $W$. In the previous example, this would mean selecting a value of age then estimating the effect of after-school experience on performance for that age. The variance of $\widehat{\theta_{X \rightarrow Y}}(W)$ is

$$
\operatorname{var}\left(\widehat{\theta_{X \rightarrow Y}}(W)\right)=\operatorname{var}\left(\hat{b}_{1}\right)+W^{2} \operatorname{var}\left(\hat{b}_{3}\right)+2 W \operatorname{cov}\left(\hat{b}_{1}, \hat{b}_{3}\right) .
$$


This equation shows that the variance of $\widehat{\theta_{X \rightarrow Y}}(W)$ is a function of the value of the moderator at which $\widehat{\theta_{X \rightarrow Y}}(W)$ is conditioned, the variance of $\hat{b}_{1}\left(\operatorname{var}\left(\hat{b}_{1}\right)\right)$, the variance of $\hat{b}_{3}\left(\operatorname{var}\left(\hat{b}_{3}\right)\right)$, and the covariance between $\hat{b}_{1}$ and $\hat{b}_{3}\left(\operatorname{cov}\left(\hat{b}_{1}, \hat{b}_{3}\right)\right)$. To estimate $\operatorname{var}\left(\widehat{\theta_{X \rightarrow Y}}(W)\right)$ use the corresponding estimates of each component: $\widehat{\operatorname{var}}\left(\hat{b}_{1}\right)$, $\widehat{\operatorname{var}}\left(\hat{b}_{3}\right)$, and $\widehat{\operatorname{cov}}\left(\hat{b}_{1}, \hat{b}_{3}\right)$. These estimates are typically calculated by any regression program. Under the null hypothesis that $\theta_{X \rightarrow Y}(W)=0$, the ratio of the estimate $\widehat{\theta_{X \rightarrow Y}}(W)$ to its standard error is $t$-distributed with $n-q-1$ degrees of freedom, where $n$ is the total number of participants and $q$ is the number of predictors in the regression equation used to estimate the coefficients and standard errors. For example, in Equation 1.12 there are three predictors, $X, W$, and $X W$, and so $q=3$. The computed $t$-statistic can be compared to a critical value from a $t$-distribution with $n-q-1$ degrees of freedom which corresponds to a specific $\alpha$-level (e.g., 0.05). If the observed statistic is more extreme than the critical value, then the null hypothesis is rejected at level $\alpha$. Alternatively, a $p$-value can be calculated based on the observed $t$-statistic. A critical value of the $t$-distribution could be used to generate a confidence interval. For example, a 100(1- $\alpha) \%$ confidence interval would be calculated as

$$
\widehat{\theta_{X \rightarrow Y}}(W) \pm t_{\alpha / 2, n-q-1}^{*} \sqrt{\widehat{\operatorname{var}}\left(\widehat{\theta_{X \rightarrow Y}}(W)\right)}
$$

where $t_{\alpha / 2, n-q-1}^{*}$ is the value in the $t$-distribution with $n-q-1$ degrees of freedom such that $\alpha / 2 \times 100 \%$ of the distribution falls above this point.

The pick-a-point approach can be used for either dichotomous or continuous moderators. The choice of points to probe in the pick-a-point approach is very clear when the moderator is dichotomous. For example, gender may be coded 0 for female and 1 for male, there is little sense of estimating the effect of $X$ on $Y$ when gender is 2 . 
However, when the moderator is continuous the choice of $W$ can be arbitrary. It has become common practice to select the mean of the moderator as well as the mean plus and minus one standard deviation (Cohen et al., 2003). However, these points may or may not be within the observed range of the data. Hayes (2018a) recommends probing at percentiles (e.g. 16th, 50th, and 84th) to guarantee that the probed points are always within the observed range of the data. There are also instances where certain points on the scale are particularly informative. For example, if the moderator is body mass index, then $18.5,25$, and 30 might be good points to probe as they indicate the boundaries between underweight, normal, overweight, and obese (World Health Organization, 2018). For detailed discussions of the pick-a-point approach for between-participant designs see Hayes and Matthes (2009), Cohen et al. (2003), Hayes (2018a), Bauer and Curran (2005), and Spiller et al. (2013).

\section{Johnson-Neyman Procedure}

The Johnson-Neyman procedure is an approach to probing interactions with continuous moderators (Johnson \& Neyman, 1936; Johnson \& Fay, 1950). Compared to the pick-a-point approach, this method reduces the arbitrariness of choosing points along the moderator. Instead this method identifies points along the moderator where the effect of the $X$ on $Y$ transitions from significant to non-significant or vice versa (i.e., boundaries of significance). The procedure uses the same point estimate and standard error as the pick-a-point approach. But rather than selecting a value of $W$ and calculating $\frac{\widehat{\theta_{X \rightarrow Y}}(W)}{\widehat{s e}\left(\theta_{X \rightarrow Y}(W)\right)}$, we select an $\alpha$-value and calculate the associated criti-

cal $t$-value, $t_{\alpha / 2, n-q-1}^{*}$. Then the Johnson-Neyman procedure solves for the values of $W$ such that the conditional effect of $X$ on $Y$ is exactly significant at the selected $\alpha$-value. Consider for example, selecting $\alpha=0.05$. The associated critical $t$-statistic 
is $t_{.025, n-q-1}^{*}$. When the ratio of the estimate of the conditional effect to its standard error is exactly equal to this $t$-value, the effect of $X$ on $Y$ will be exactly significant where $p=0.05$. This point is found by setting the ratio of $\widehat{\theta_{X \rightarrow Y}}(W)$ to its standard error equal to the critical $t$ and solving for $W$.

Solving for $W$ involves finding the roots of a quadratic equation. Sometimes the solutions are imaginary (a function of $\sqrt{-1}$ ) or outside of the range of the observed data; I do not recommend interpreting these solutions. By finding the boundaries of significance, the Johnson-Neyman method allows us to understand the patterns of significance across the range of the moderator, rather than arbitrarily selecting points like in the simple slopes method. The Johnson-Neyman method can only be used for continuous moderators. In the example, the Johnson-Neyman procedure could be used to find the ages for which after-school experience has a significant (or non-significant) effect on performance. For example, the Johnson-Neyman procedure could reveal that 8 is the boundary of significance, meaning that for students who are 8 years old the relationship between after-school experience and performance is exactly significant. By examining the patterns of significance on each side of the boundary we could see that students younger than 8 did not show a significant effect of after-school experience on performance, but for students older than 8 there is a significant positive effect of after-school experience on science performance.

Moderation analysis is a key part of understanding moderated mediation, which combines mediation and moderation together to allow for an indirect effect to depend on a moderator. Much of the language from moderation analysis is shared with conditional process analysis. Next, I discuss how conditional process analysis is conducted in between-subjects designs. 


\subsection{Conditional Process Analysis}

Conditional process analysis, or moderated mediation analysis, is used when we are interested in if an indirect effect is moderated. This occurs when the effect of independent variable $X$ on an outcome $Y$ through some mediator $M$ depends on another variable $W$. It may be that for some individuals the indirect effect is stronger than for other individuals. An indirect effect can be positive for some and negative for others. For example, assume we are interested in understanding why certain people believe in climate change and others do not. Recently, research has suggested that including estimates of uncertainty due to sampling variability (e.g., confidence intervals) along with the results of climate change research might help individuals trust the research more (Joslyn \& LeClerc, 2016). Perhaps we believe that this would work for more liberal individuals, but may actually be counter productive for those who are more conservative. We could do a study where we randomly assign individuals to read an article by a scientist about climate change that either does or does not include uncertainty estimates $(X)$. Next, each individual rates how much they trust the scientist who wrote the article $(M)$ and how much they believe in climate change $(Y)$. We also collect political orientation on a continuous scale (conservative - liberal; $W$ ). We believe that among those who are more liberal, including uncertainty estimates will improve trust in the scientists, which will then increase belief in climate change. But we also believe that among those who are more conservative, including uncertainty estimates will decrease trust in the scientist (perhaps because uncertainty estimates contribute to the belief that the scientists are unreliable), then this decreased trust will actually decrease belief in climate change in comparison to those who did not view uncertainty estimates. This is an example where the indirect effect might be 
positive for those who scored higher on political orientation (liberal), but would be negative for those who scored lower on political orientation (conservative). This is the type of research question which can be answered using conditional process analysis.

\subsubsection{A General Conditional Process Model}

To conduct a conditional process analysis, we need to identify the paths in the mediation process which are expected to be moderated. Often we expect the moderator intervenes on the relationship between the independent variable and the mediator. This is called first-stage moderated mediation because only the first stage (the $X$ to $M$ relationship) is moderated. If the moderator only affects the relationship between $M$ and $Y$ this is second-stage moderated mediation. The moderator can affect both the first and second stage. Additionally, moderation of the direct effect can be included in any of these models.

Early approaches to moderated mediation used a very general model where the moderator is allowed to moderate the relationship between $X$ and $M$, the relationship between $M$ and $Y$, and the direct effect of $X$ on $Y$ (Fairchild \& MacKinnon, 2009; Muller et al., 2005). In this framework, regression models are setup such that each effect is conditional on a common moderator.

$$
\begin{aligned}
Y_{i} & =c_{0}+f_{c}\left(W_{i}\right) X_{i}+c_{2} W_{i}+e_{Y_{i}} \\
M_{i} & =a_{0}+f_{a}\left(W_{i}\right) X_{i}+a_{2} W_{i}+e_{M_{i}} \\
Y_{i} & =c_{0}^{\prime}+f_{c^{\prime}}\left(W_{i}\right) X_{i}+f_{b}\left(W_{i}\right) M_{i}+b_{2} W_{i}+e_{Y_{i}^{\prime}}
\end{aligned}
$$

where 


$$
\begin{aligned}
& f_{c}\left(W_{i}\right)=c_{1}+c_{3} W_{i} \\
& f_{a}\left(W_{i}\right)=a_{1}+a_{3} W_{i} \\
& f_{c^{\prime}}\left(W_{i}\right)=c_{1}^{\prime}+c_{3}^{\prime} W_{i} \\
& f_{b}\left(W_{i}\right)=b_{1}+b_{3} W_{i}
\end{aligned}
$$

In this model, the total effect of $X$ on $Y$ is a linear function of $W, f_{c}\left(W_{i}\right)$. Similarly, the effect of $X$ on $M$ is a linear function of $W, f_{a}\left(W_{i}\right)$, the effect of $M$ on $Y$ is a linear function of $W, f_{b}\left(W_{i}\right)$, and the effect of $X$ on $Y$ controlling for $M$ is a linear function of $W, f_{c^{\prime}}\left(W_{i}\right)$. A path diagram of this model is included in Figure 1.4. If all of the coefficients $c_{3}, a_{3}, c_{3}^{\prime}$, and $b_{3}$ were zero, then this model would simplify to a mediation model where $W$ is a covariate in all the equations. The indirect effect is the product of the effect of $X$ on $M$ and the effect of $M$ on $Y$. Both of these effects are conditional, and so the indirect effect will also be conditional. I denote the conditional indirect effect as $\theta_{X \rightarrow M \rightarrow Y}(W)$.

$$
\begin{aligned}
\theta_{X \rightarrow M \rightarrow Y}(W) & =\theta_{X \rightarrow M}(W) \theta_{M \rightarrow Y}(W) \\
& =f_{a}(W) f_{b}(W) \\
& =\left(a_{1}+a_{3} W\right)\left(b_{1}+b_{3} W\right) \\
& =a_{1} b_{1}+\left(a_{1} b_{3}+a_{3} b_{1}\right) W+a_{3} b_{3} W^{2}
\end{aligned}
$$

Though each component of the indirect effect is a linear function of $W$, the indirect effect is a quadratic function of $W$. In this general model, there is no need to decide which paths are moderated. However this model can include potentially unnecessary 
parameters, which may reduce power to detect moderated mediation effects and make interpretation more complex. Additionally, inference about moderated mediation with these models can be quite difficult because of the nonlinear nature of the indirect effect when the moderator is continuous. Some have suggested that if either $\hat{a}_{1} \hat{b}_{3}+\hat{a}_{3} \hat{b}_{1}$ or $\hat{a}_{3} \hat{b}_{3}$ are significantly different from zero you can claim moderated mediation (Muller et al., 2005; Fairchild \& MacKinnon, 2009). However this relies on a fairly piecewise logic that is not completely satisfactory for the same reasons that the Baron and Kenny (1986) causal steps method is not satisfactory.

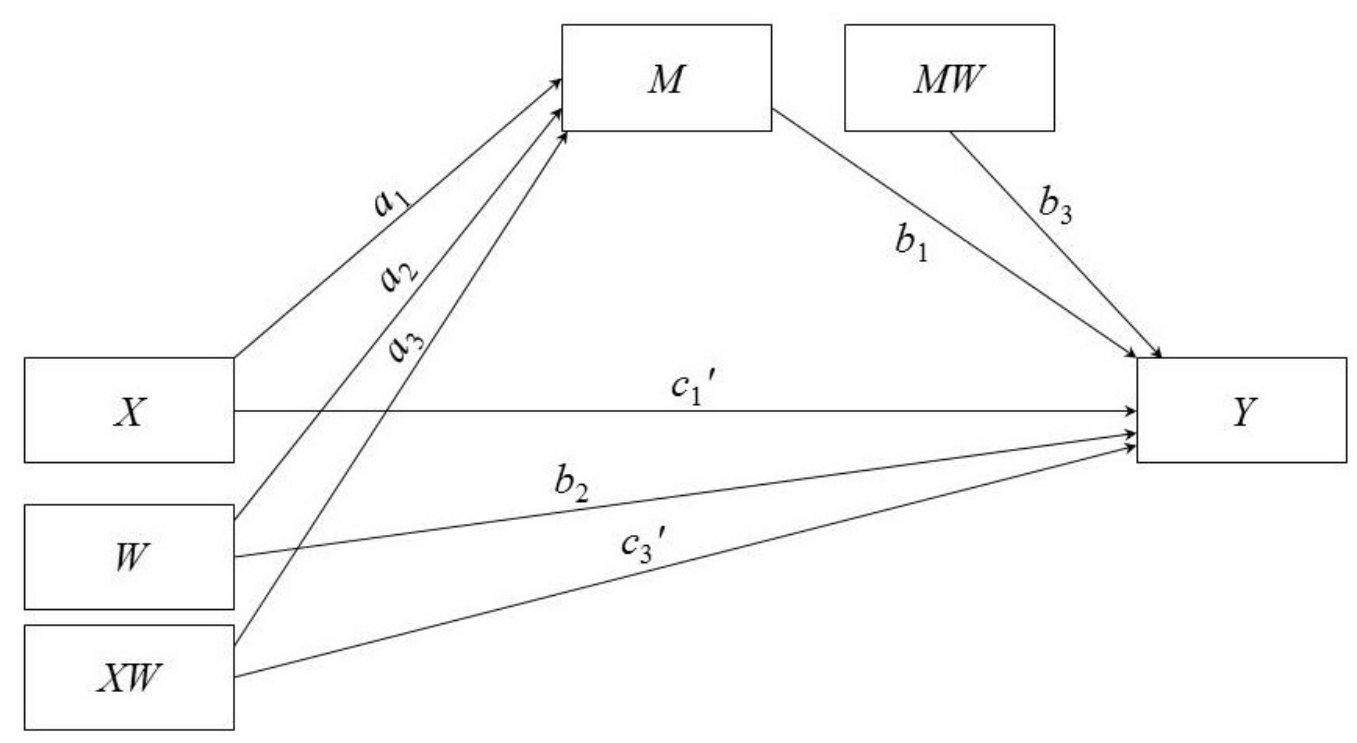

Figure 1.4: Path Diagram for Conditional Process Model for Between Subjects Design 


\subsubsection{First-Stage Conditional Process Model}

The model described in Section 1.5.1 is quite general. It would be more parsimonious to select a model where some but not all of the paths in the mediation are moderated. Such models are particularly useful if the theory is clear about which paths are expected to be moderated. Preacher et al. (2007) and Edwards and Lambert (2007) proposed a variety of models with a limited number of paths being moderated. Based on the choice of model, different regression models are used to define the effects of interest. For example, a first-stage conditional process model allows the relationship between $X$ and $M$ to be conditional on $W$. The relationship between $M$ and $Y$ is not affected by $W$. The direct effect could be conditional on $W$ or not. For this type of model the equation for the mediator matches that of the general model:

$$
M_{i}=a_{0}+\left(a_{1}+a_{3} W_{i}\right) X_{i}+a_{2} W_{i}+e_{M_{i}}
$$

The conditional effect of $X$ on $M$ is $\theta_{X \rightarrow M}(W)=a_{1}+a_{3} W_{i}$. If the direct effect is moderated, the equation for the outcome is

$$
Y_{i}=c_{0}^{\prime}+\left(c_{1}^{\prime}+c_{3}^{\prime} W_{i}\right) X_{i}+b_{1} M_{i}+b_{2} W_{i}+e_{Y_{i}^{\prime}}
$$

In this model the effect of $M$ on $Y$ does not depend on $W$. It is $b_{1}$. The direct effect is conditional $W: c_{1}^{\prime}+c_{3}^{\prime} W$. In this model, the indirect effect is the product of the effect of $X$ on $M$ and the effect of $M$ on $Y:\left(a_{1}+a_{3} W\right) b_{1}=a_{1} b_{1}+a_{3} b_{1} W$. Hayes (2015) named the parameter $a_{3} b_{1}$ the index of moderated mediation, for this model. When the index is zero, the function defining the conditional indirect effect is constant with respect to $W$, therefore there is no moderated mediation. Inference 
about this parameter provides a direct test of moderated mediation in this first stage model.

If the direct effect is not moderated, the equation for the outcome is

$$
Y_{i}=c_{0}^{\prime}+c^{\prime} X_{i}+b_{1} M_{i}+e_{Y_{i}^{\prime}}
$$

Just like Equation 1.25, the effect of $M$ on $Y$ is not conditional and is represented by the parameter $b_{1}$. Again the conditional indirect effect is $a_{1} b_{1}+a_{3} b_{1} W$, and the index of moderated mediation is $a_{3} b_{1}$. But now, the direct effect is not conditional: it is $c^{\prime}$. Note that though it may appear that the indirect effect does not depend on whether you allow the direct effect to be moderated or not, the estimates of $b_{1}$ will depend on whether you allow the direct effect to be moderated. This means that it's important to consider which model aligns closest with the theory you are testing.

\subsubsection{Second-Stage Conditional Process Model}

Another popular model is one which allows the relationship between $M$ and $Y$ to be moderated by $W$. Again, the direct effect can be conditional or unconditional. The model for the mediator is the same as for the unmoderated mediation model.

$$
M_{i}=a_{0}+a X_{i}+e_{M_{i}}
$$

The effect of $X$ on $M$ is $a$ and does not depend on the moderator $W$. If the direct effect is moderated, the equation for the outcome is the same as that from the general model:

$$
Y_{i}=c_{0}^{\prime}+\left(c_{1}^{\prime}+c_{3}^{\prime} W_{i}\right) X_{i}+\left(b_{1}+b_{3} W_{i}\right) M_{i}+b_{2} W_{i}+e_{Y_{i}^{\prime}}
$$

The effect of $M$ on $Y$ is a linear function of $W, b_{1}+b_{3} W$. The direct effect is also conditional: $c_{1}^{\prime}+c_{3}^{\prime} W$. The indirect effect is the product of the effect of $X$ on $M$ and 
the effect of $M$ on $Y$. The indirect effect is $a\left(b_{1}+b_{3} W\right)=a b_{1}+a b_{3} W$. The parameter $a b_{3}$ is the index of moderated mediation for this model. Inference on this parameter provides a direct test of moderated mediation in the second-stage conditional process model.

If the direct effect is not moderated, the equation for the outcome is

$$
Y_{i}=c_{0}^{\prime}+c^{\prime} X_{i}+\left(b_{1}+b_{3} W_{i}\right) M_{i}+b_{2} W_{i}+e_{Y_{i}^{\prime}}
$$

Just like the previous model, the effect of $M$ on $Y$ is conditional: $b_{1}+b_{3} W$. The conditional indirect effect is the same as when the direct effect is moderated, $a b_{1}+$ $a b_{3} W$. The index of moderated mediation is still $a b_{3}$. The direct effect is $c^{\prime}$ and so does not depend on $W$. The $b_{1}$ and $b_{3}$ parameter estimates will depend on whether or not the direct effect is allowed to be conditional.

\subsubsection{Methods of Inference}

Inference for moderated mediation has evolved over time and continues to develop as this area is relatively new. Baron and Kenny (1986) described the first-stage moderated mediation model. To claim moderated mediation, they required a significant interaction between $X$ and $W$ predicting $Y$ ( $c_{3}$ in Equations 1.16). Additionally, there must be a significant interaction between $X$ and $W$ predicting $M, a_{3}$ from Equation 1.24. The last requirement is that $b_{1}$ in Equation 1.25 be significantly different zero. This is very similar to the causal steps method for establishing evidence for mediation, and all the criticisms of the causal steps method apply.

Hayes (2015) showed that a null hypothesis of no moderated mediation would imply that the index of moderated mediation is zero. When the index of moderated mediation is zero, the indirect effect is constant across the range of the moderator. 
Hayes (2015) proposes that inference about moderated mediation should be done using bootstrap confidence intervals for the index of moderated mediation specific to the model being investigated.

Bootstrapping is a nonparametric method which uses the sample as an empirical estimate of the distribution of the measured variables in the population (Efron \& Tibshirani, 1993). Bootstrapping repeatedly generates bootstrap samples of the same size as the original sample by sampling with replacement from the original sample. With each bootstrap sample, estimates of the parameters of interest are calculated, and this process is repeated many many times. Specifically for moderated mediation, for each bootstrap sample an estimate of the index of moderated mediation is calculated, resulting in a bootstrap distribution of estimates of the index of moderated mediation. This distribution can be used to calculate an estimate of the standard error of the index, or percentiles of the distribution can be used to generate a confidence interval for the indirect effect. Much research in the between-subjects domain suggests that percentile bootstrapping methods perform very well for inference for indirect effects (Williams \& MacKinnon, 2008; MacKinnon et al., 2004; Hayes \& Scharkow, 2013; Biesanz et al., 2010). Bootstrap confidence intervals are similarly useful in this instance because the index of moderated mediation is the product of two normally distributed variables, the distribution of which is not always normal. Bootstrapping estimates the sampling distribution of the index of moderated mediation, allowing for valid inference regardless of the shape of the distribution. 


\section{Chapter 2: Mediation and Moderation in Two-Instance Repeated-Measures Designs}

The body of research on mediation and moderation in two-instance repeatedmeasures designs is quite small in comparison to the body of research on these analyses for between-subjects designs. The focus of this dissertation is on conditional process analysis in two-instance repeated-measures designs. In this chapter, I describe developments in mediation and moderation in two-instance repeated-measures designs to establish how these methods might be combined to implement conditional process analysis.

\subsection{Two-Instance Repeated-Measures Designs}

A two-instance repeated-measures design manifests itself in many different ways. This design is used frequently in experimental social science when each participant responds under two different conditions or situations. Consider, for example, studying how stereotypes about computer science impacts students interest in studying computer science. Cheryan, Meltzoff, and Kim (2011) had students use a virtual avatar to tour two different classrooms for computer classes. One classroom had stereotypical decorations (e.g., Star Trek figurine, technology magazines), whereas the other classroom was decorated neutrally (e.g., desk lamp, general magazines). All participants 
toured both classrooms and rated how interested they were in taking each class. This is an example of a two-instance repeated-measures design.

Another common two-instance repeated-measures design is a longitudinal design with only two measurement occasions. Imagine a therapist treating a variety of participants with a new type of treatment for bipolar disorder. In order to discern whether or not the treatment is helping, you might measure all the participants on their bipolar symptomatology before they start treatment and again after 12 weeks of treatment. Often times when analyzing repeated-measures designs, it is important to differentiate longitudinal and within-subjects designs based on whether or not time is the factor that differentiates the measurement instances. However, in the case of two-instance repeated-measures designs, there is no need to differentiate these two cases. Two time point longitudinal designs can be treated the same as a two-condition within-subjects design. The type of analysis described in this chapter would also be appropriate for designs where all participants undergo the same treatment and are measured at two occasions and designs where all participants are measured in two different experimental conditions.

Two-instance repeated-measures designs differ from between-subjects designs in that each individual is measured at two different instances, whereas in the betweensubjects design, each individual experiences only one treatment or is measured on the $X$ variable, and the groups of individuals experiencing each treatment are compared to each other. In repeated-measures designs, each person's response in one instance is compared to their own response in the other instance. A major part of setting up the model for mediation in the two-instance repeated-measures design is maintaining and taking into account the connection between the responses from each individual. 


\subsection{Mediation}

In order to set up the model, each individual will need to have four measurements. First, there will be two measurements of the outcome $Y$ for each individual $i$. Call these $Y_{1 i}$ and $Y_{2 i}$, where $Y_{1 i}$ is individual $i$ 's response on the outcome in Instance 1, and $Y_{2 i}$ is individual $i$ 's response on the outcome in Instance 2. Similarly, we will have two measurements of the mediator, one for each instance. Denote $M_{1 i}$ and $M_{2 i}$ to be individual $i$ 's responses on the mediator in Instance 1 and 2 respectively. Instance 1 is not necessarily the "first" instance and Instance 2 is not necessarily the "second." Rather the labels 1 and 2 can be assigned by the researcher, so long as they are consistent across the outcome and the mediator. Assume we have $N$ individuals in the study, so that $i=1, \ldots, N$.

\subsubsection{A Path-Analytic Model}

In mediation analysis, we typically begin by modeling the overall effect of the instance on the outcome $Y$. In the between-subjects case we regress the outcome onto the treatment predictor $X$. In the two-instance repeated-measures case there are two observations for each individual, one in each treatment instance, and that

dependency must be taken into account. We can take the dependency into account by setting up an intercept-only model for each of the outcomes in a path analysis.

$$
\begin{aligned}
& Y_{1 i}=c_{1}+e_{Y_{1 i}} \\
& Y_{2 i}=c_{2}+e_{Y_{2 i}}
\end{aligned}
$$


The outcome in each instance has a specific population mean, $c_{1}$ for Instance 1 and $c_{2}$ for Instance 2. Each individual has their own deviations around the population means. In Instance 1, individual $i$ 's measurement on $Y_{1 i}$ deviates from $c_{1}$ by $e_{Y_{1 i}}$ and in Instance 2, $Y_{2 i}$ deviates from $c_{2}$ by $e_{Y_{2 i}}$. Assume that the errors are distributed with means of zero and with some variance-covariance matrix $\Sigma_{Y}$ where $\sigma_{Y_{1}}^{2}$ is the variance of the $e_{Y_{1 i}}$ 's and $\sigma_{Y_{2}}^{2}$ is the variance of the $e_{Y_{2 i}}$ 's. I denote the correlation among the two errors as $\rho_{Y}$. This correlation is what differentiates this model from the between-subjects model and accounts for the dependency among responses from the same person. In a between-subjects model, observations from Instance 1 are assumed to be independent from observations from Instance 2 (i.e., $\rho_{Y}=0$ ). Here however, observations from the same person are allowed to be correlated.

A parameter that reflects the overall effect of the treatment on the outcome $Y$ is the difference in the two means $c_{1}$ and $c_{2}$. Taking the difference between the Equations 2.1 and 2.2 gives a new equation that includes this parameter:

$$
Y_{2 i}-Y_{1 i}=c_{2}-c_{1}+e_{Y_{2 i}}-e_{Y_{1 i}}=c+e_{Y_{i}}
$$

To simplify notation, I denote $c=c_{2}-c_{1}$; This is the parameter which reflects the difference in the means on the outcome between the two instances. This is the total effect of instance on the outcome. The difference between the error terms $e_{Y_{i}}=$ $e_{Y_{2 i}}-e_{Y_{1 i}}$ will have mean zero and a new variance which is a function of $\sigma_{Y_{1}}^{2}, \sigma_{Y_{2}}^{2}$, and $\rho_{Y}$. A test on $c$ using this intercept only regression method is equivalent to a paired $t$-test on the outcome measures. Both these tests require an additional assumption that the errors are normally distributed or reliance on the Central Limit Theorem. 
The indirect effect will be a function of the effect of instance on the mediator and the effect of the mediator on the outcome controlling for the instance. The first of these can be defined in a very similar way to the total effect. The total effect $c$ is the effect of the instance on the outcome. Similar models can be used to define the effect of the instance on the mediators, which I will call $a$. Define a model for the mediator in each instance. Each mediator has its own mean $a_{1}$ and $a_{2}$ respectively for Instance 1 and Instance 2. The errors are assumed have mean zero and variance covariance matrix $\Sigma_{M}$ where $\sigma_{M_{1}}^{2}$ is the variance of the $e_{M_{1 i}}$ 's and $\sigma_{M_{2}}^{2}$ is the variance of the $e_{M_{2 i}}$ 's. The correlation among the two errors can be expressed as $\rho_{M}$.

$$
\begin{aligned}
M_{1 i} & =a_{1}+e_{M_{1 i}} \\
M_{2 i} & =a_{2}+e_{M_{2 i}} \\
M_{2 i}-M_{1 i} & =a_{2}-a_{1}+e_{M_{2 i}}-e_{M_{1 i}}=a+e_{M_{i}}
\end{aligned}
$$

Again, taking the difference between equations for each instance results in a single equation which includes the parameter of interest, $a$, the effect of instance on the mediator. Just as with the total effect, a test on a using this method is equivalent to a paired $t$-test on the mediator measures. Again, the hypothesis test for $a$ either through regression or a paired $t$-test requires the additional assumption that the errors are normally distributed.

A parameter that reflects the effect of the mediator on the outcome is still needed. Judd et al. (2001) use the following models where the outcome in Instance 1 is a linear function of the mediator in Instance 1. Similarly for Instance 2, the outcome in Instance 2 is a linear function of the mediator in Instance 2. One of the assumptions of 
this model is that the mediator from the other instance does not predict the outcome above and beyond the mediator from the same instance. For example, in a model of the outcome from Instance 1, the mediator from Instance 2 does not predict this outcome above and beyond the mediator from Instance 1. This is made explicit in the equations below.

$$
\begin{aligned}
& Y_{1 i}=c_{1}^{\prime *}+b_{11} M_{1 i}+e_{Y_{1 i}^{\prime}} \\
& Y_{2 i}=c_{2}^{* *}+b_{21} M_{2 i}+e_{Y_{2 i}^{\prime}}
\end{aligned}
$$

Similar to the previous equations, $c_{1}^{*}$ and $c_{2}^{*}$ are intercepts, and the subscript denotes which instance the outcome is measured in. The slopes $b_{11}$ and $b_{21}$ quantify the relationship between the mediator and the outcome in their respective instances denoted by the first subscript. Assume that the errors $e_{Y_{1 i}^{\prime}}$ and $e_{Y_{2 i}^{\prime}}$ have means of zero and variance covariance matrix $\Sigma_{Y^{\prime}}$, variances $\sigma_{Y_{1}^{\prime}}^{2}, \sigma_{Y_{2}^{\prime}}^{2}$, and correlation $\rho_{Y^{\prime}}$.

In these models there is not one but two parameters that estimate the relationship between the mediator and the outcome variable, $b_{11}$ and $b_{21}$. Judd et al. (2001) outline how you can assume that these two parameters are equal and estimate a single parameter, accordingly. They also describe a method which does not require this assumption, but rather to uses the average of these two parameters to represent the relationship between the mediator and the outcome. Additionally, the model includes a parameter that reflects the difference between the two slopes $b_{11}$ and $b_{21}$ in order to provide additional information about whether these slopes differ across instance. Take the difference between Equation 2.8 and Equation 2.7 and applying a $45^{\circ}$ rotation so the equation includes the parameters of primary interest: 


$$
\begin{aligned}
Y_{2 i}-Y_{1 i} & =c_{2}^{\prime *}-c_{1}^{\prime *}+b_{21} M_{2 i}-b_{11} M_{1 i}+e_{Y_{2 i}^{\prime}}-e_{Y_{1 i}^{\prime}} \\
& =c_{2}^{\prime *}-c_{1}^{\prime *}+\frac{b_{21}+b_{11}}{2}\left(M_{2 i}-M_{1 i}\right)+\left(b_{21}-b_{11}\right) \frac{M_{2 i}+M_{1 i}}{2}+e_{Y_{2 i}^{\prime}}-e_{Y_{1 i}^{\prime}} \\
& =c^{\prime *}+b\left(M_{2 i}-M_{1 i}\right)+d \frac{M_{2 i}+M_{1 i}}{2}+e_{Y_{i}^{\prime}}
\end{aligned}
$$

To simplify notation, let

$$
\begin{aligned}
c^{\prime *} & =c_{2}^{\prime *}-c_{1}^{\prime *} \\
b & =\frac{b_{21}+b_{11}}{2} \\
d & =b_{21}-b_{11} \\
e_{Y_{i}^{\prime}} & =e_{Y_{2 i}^{\prime}}-e_{Y_{1 i}^{\prime}} .
\end{aligned}
$$

The difference between the outcomes is modeled as a linear function of the difference between the mediators and the average of the mediators. Additionally, the weights of these predictors align with the parameters of primary interest. The coefficient $\frac{b_{21}+b_{11}}{2}$ is the effect of the mediator on the outcome averaged over instances, and the coefficient $\left(b_{21}-b_{11}\right)$ is the difference in the relationship between the mediator and the outcome across instances. The intercept of this equation, $c_{2}^{\prime *}-c_{1}^{\prime *}$, is the difference in the outcomes for individuals with no difference in the mediators $\left(M_{2 i}-M_{1 i}=0\right)$ and for whom the average of the mediators is zero $\left(\left(M_{2 i}+M_{1 i}\right) / 2=0\right)$. The intercept may not be interpretable, depending on the scaling of the mediator.

In between-subjects mediation, the direct effect is the difference between the outcomes for individuals with no difference in the mediators. Because the model for 
within-subjects designs also includes the average of the mediators, a reasonable measure of the direct effect might be conditional on the sample mean of the average of the mediators. This can be achieved by mean centering the average mediator variable

$$
\begin{aligned}
Y_{2 i}-Y_{1 i} & =c^{\prime *}+b\left(M_{2 i}-M_{1 i}\right)+d \frac{M_{2 i}+M_{1 i}}{2}+e_{Y_{i}^{\prime}} \\
& =c^{\prime *}+d \frac{\overline{M_{2 \cdot}+M_{1 \cdot}}}{2}+b\left(M_{2 i}-M_{1 i}\right)+d\left(\frac{M_{2 i}+M_{1 i}}{2}-\frac{\overline{M_{2 \cdot}+M_{1 \cdot}}}{2}\right)+e_{Y_{i}^{\prime}} \\
& =c^{\prime}+b\left(M_{i 2}-M_{i 1}\right)+d\left(\frac{M_{i 2}+M_{i 1}}{2}-\frac{\overline{M_{2 \cdot}+M_{1 \cdot}}}{2}\right)+e_{Y_{i}^{\prime}}
\end{aligned}
$$

I denote the sample mean over all individuals $i$ of the average of the mediators over the two instances, as $\frac{\overline{M_{2}+M_{1}}}{2}=\frac{1}{N} \sum_{i=1}^{N} \frac{M_{2 i}+M_{1 i}}{2}$. In addition, the equations are simplified such that

$$
c^{\prime}=c_{2}^{* *}-c_{1}^{\prime *}+d \frac{\overline{M_{2 \cdot}+M_{1}}}{2}
$$

The coefficient $c^{\prime}$ is a conditional direct effect (i.e., the effect of instance on the outcome controlling for the mediator, conditional on the sum of the mediators being at the sample average). The coefficient $b$ is the parameter for the average effect of the mediator on the outcome, and $d$ is the difference in the two effects of the mediator on the outcome.

All of these equations can be put together into a path diagram similar to the one in Figure 1.1. However, because the intercepts are important in these equations they are represented in the paths from the triangle to the outcome variable in the model. Figure 2.1 provides a path diagram for the two-instance repeated-measures mediation model. 
From Figure 2.1 it is clear that the indirect effect is the product of the two parameters $a$ and $b$. It is also clear that $c^{\prime}$ is the direct effect and $c$ is the total effect. So we can use linear regression to estimate these parameters in order to get estimates of all of the effects of interest in mediation in this two-instance repeated measures design.
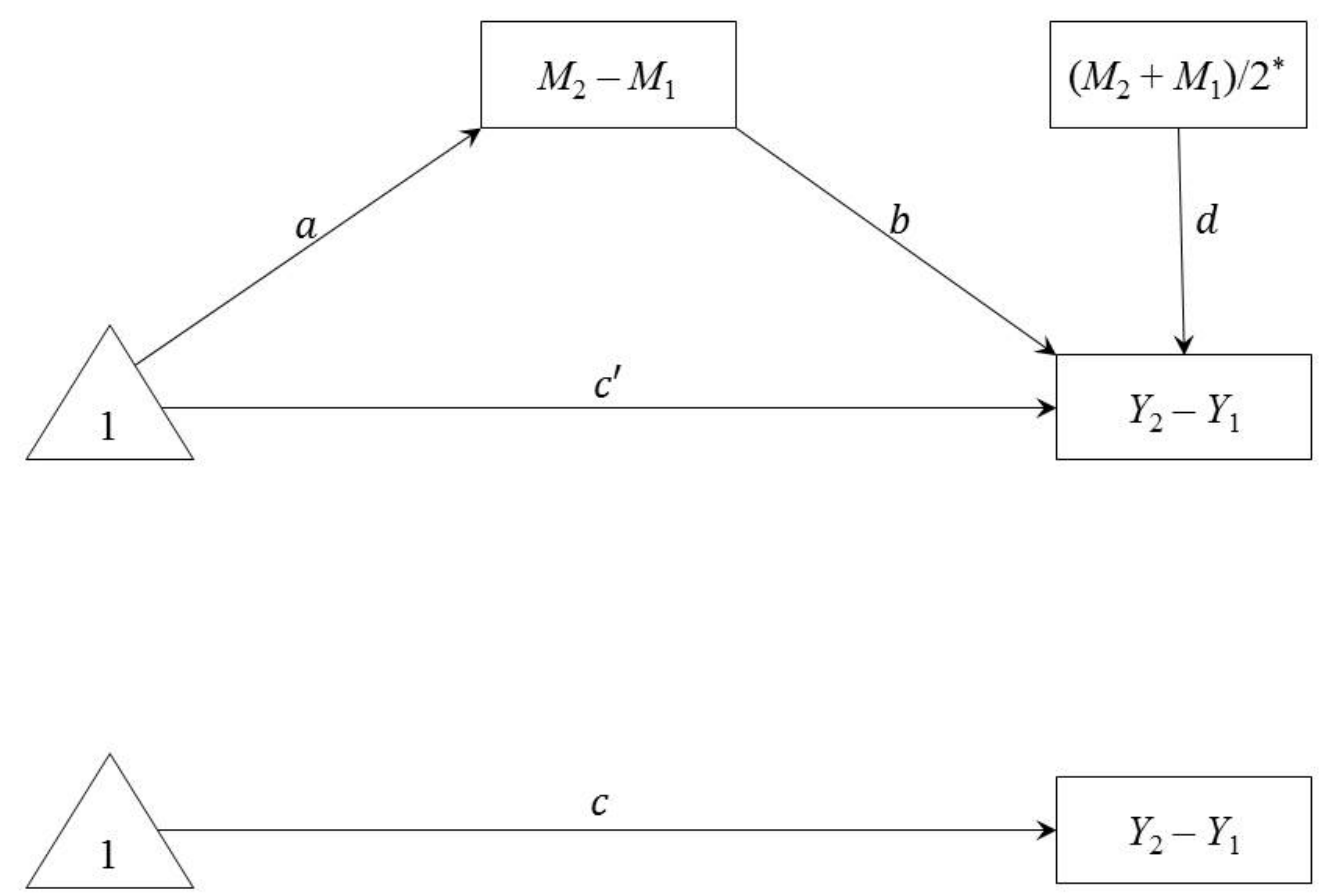

Figure 2.1: Path Diagram for Mediation Model for Two-Instance Repeated-Measures Design

* indicates grand-mean centered. 


\subsubsection{Estimation and Inference}

Equations 2.3, 2.6, and 2.14 can be estimated separately using ordinary least squares (OLS) regression analysis. Alternatively, structural equation modeling (SEM) can be used to estimate all these parameters simultaneously. The estimates of the coefficients will be the same whether regression or SEM is used. However, the estimates of the standard errors will differ by method, as structural equation modeling uses asymptotic standard error estimates and OLS does not (Hayes, 2018a; Hayes, Montoya, \& Rockwood, 2017).

Either method will provide estimates of each of the coefficients in Equations 2.3, 2.6, and 2.14. In particular, we can use the estimates $\hat{a}, \hat{b}, \hat{c}$, and $\hat{c}^{\prime}$ for the total, direct and indirect effects. The total effect estimate is $\hat{c}$ and the direct effect estimate is $\hat{c}^{\prime}$. As the indirect effect is the product of $a$ and $b$, the estimate of the indirect effect is $\hat{a} \hat{b}$.

The direct and total effect are regression coefficients and so inference for these can be conducted using the typical methods from regression or structural equation modeling, whichever method was used to estimate the coefficients. As mentioned previously, these tests rely on the assumption that the error terms in the equations are normally distributed. Hypothesis tests for these coefficients can be conducted by taking the ratio of the estimated coefficient to its estimated standard error and comparing this to a $t$-distribution. Under the null hypothesis that the parameter is

zero, this ratio (e.g., $\frac{\hat{c}}{\widehat{s \hat{e}_{\hat{c}}}}$ ) is $t$-distributed with $n-q-1$ degrees of freedom where $q$ is the number of predictors in the regression equation. Similarly, a confidence interval for each of these coefficients can be constructed using the estimated standard error and a critical $t$-value. For $95 \%$ confidence interval use the value $t_{.025, d f}$ which denotes the 
value of the $t$ distribution with $d f$ degrees of freedom where $2.5 \%$ of the distribution lies above that value. The confidence interval would be $\hat{c} \pm t_{.025, N-1} \times \widehat{s e}_{\hat{c}}$ for the total effect and $\hat{c}^{\prime} \pm t_{.025, N-3} * \widehat{s e}_{\hat{c}^{\prime}}$ for the direct effect. Note in the model including $c$ (Equation 2.3), the number of predictors is zero, as it is an intercept only model. The model for the direct effect includes two predictors, so $q=2$ and $d f=N-3$.

Inference for the indirect effect can be conducted in a variety of ways. Judd et al. (2001) recommended a piece-meal test, such that evidence of mediation is established if $\hat{c}$ is significantly different from zero, $\hat{a}$ is significantly different from zero, and $\hat{b}$ is significantly different from zero. Each of these tests requires an assumption that the error terms involved are normally distributed. However, this method has the same issues that the causal steps method has in the between subjects case (Montoya \& Hayes, 2017). In particular, conditioning on a significant total effect $\hat{c}$ is unnecessary, and a piece-meal test of the indirect effect is less parsimonious than a single test on an estimate of the indirect effect, $\widehat{a b}$. Finally, this method does not provide a point estimate or confidence interval for the indirect effect, and so other methods would be preferable. Some other methods to consider would be bootstrapping or the Monte Carlo confidence interval.

Bootstrapping would be conducted as described in Chapter 1. When the resampling is done in this repeated-measures case, the resampling must be done at the individual level. If individual observations from the same person were broken up, this would not properly account for the dependencies in the data. All of the observations from a single individual must be sampled all together, meaning that each case that is resampled should include four measurements: the outcome in each instance and 
the mediator in each instance. Given a bootstrap distribution of the indirect effect, confidence intervals can be generated based on percentiles of the distribution.

Another method for conducting inference on the indirect effect is the Monte Carlo confidence interval method. This method relies on the normal distribution of the estimates of the individual paths in the indirect effect $\hat{a}$ and $\hat{b}$, then uses simulation methods to generate a distribution of indirect effects which can be used to create confidence intervals. From the original analysis, the estimates of the $a$-path and $b$-path and their standard errors are all saved. Next a large number of samples (e.g., 5,000) from two different normal distributions is generated: one set of values are simulated from a normal distribution with mean $\hat{a}$ and standard deviation $\widehat{s e_{\hat{a}}}$. The second simulates random numbers from a normal distribution with mean $\hat{b}$ and standard deviation $\widehat{\operatorname{se}_{\hat{b}}}$. These sampled values are multiplied together, resulting in an estimated sampling distribution of indirect effects. From this distribution we can use the 2.5 th and 97.5 th percentile to generate a $95 \%$ confidence interval. Previous research in between-subjects mediation suggests that this method works well (Hayes \& Scharkow, 2013; Selig \& Preacher, 2009).

There are a variety of other methods for conducting inference for the indirect effect (e.g., the joint significance test, the Sobel test, the distribution of the product method, Bayesian credible intervals). I describe bootstrap and Monte Carlo confidence intervals here because they both respect the nonnormality of the indirect effect, they can be used to provide a point estimate and confidence interval for the indirect effect, and they are both available in an easy to use tool for SPSS and SAS called MEMORE (Montoya \& Hayes, 2017). 


\subsection{Moderation}

Moderation analysis and probing have been widely described in between-participant designs, resulting in an increasing use of methods and new tools that made probing interactions easier (Hayes \& Matthes, 2009; Hayes, 2013; McCabe, Kim, \& King, 2018). Recent advances in probing have generalized these methods to multilevel modeling and latent curve analysis (Bauer \& Curran, 2005; Preacher, Curran, \& Bauer, 2006). However, the two-instance repeated-measures design has been neglected when it comes to testing and probing moderation. Judd et al. (1996) and Judd et al. (2001) described methods for estimating and testing if there is an interaction in two-instance repeated-measures designs, and these methods have been used across areas of psychological science to investigate questions of moderation. For example, Manikandan et al. (2016) surveyed women during two phases of their menstrual cycle and found that emotional regulation moderated the effect of menstrual phase on perceived control over anxiety-related events, but emotion regulation did not moderate the effect of phase on menstrual symptom severity. In another example, among students with math difficulties, those with higher working memory capacity benefited more from strategy training (pre-to-posttest) than those with lower working memory capacity (Powell, Cirino, \& Malone, 2017).

In this section, I will review the methods described by Judd et al. (1996, 2001) for estimating a model to test if there is moderation of the effect of a repeated measures factor by some between-participant variable. The moderator is assumed to be constant across instance within an individual. Then I will describe the methods for probing an interaction using both the simple slopes method and the Johnson-Neyman procedure (Montoya, in press). 
To do this analysis, the data will need to be in wide form: each row of the dataset represents a participant, and each measurement from each instance is saved in a different variable. For example there must be a measure of the outcome in Instance 1, a measure of the outcome in Instance 2, and a measure of the moderator. With the data in this form, each of the rows of the dataset are independent. However, there is no instance variable in the dataset. Without this variable there is no way to regress $Y$ onto $X, W$, and $X W$ (as described in Section 1.4). Using the data in wide form, I will describe how to estimate and probe interactions between instance and a between-participant variable in predicting an outcome variable.

\subsubsection{Testing an Interaction}

Many researchers describe and understand interactions as differences in slopes. A slope describes the linear relationship between the predictor and the outcome. An interaction means that this slope depends on some other variable, a moderator. Judd et al. (1996, 2001) use this idea of varying slopes to outline a method for testing interaction between a between-participant variable and a repeated-measures factor. This procedure begins with a model of the outcome variable $Y$ predicted by $W$, the between-participant variable, where the regression weights for this model are allowed to vary by instance. In the case of two instances there are two models, one for each outcome variable:

$$
\begin{aligned}
& Y_{1 i}=b_{10}+b_{11} W_{i}+e_{1 i} \\
& Y_{2 i}=b_{20}+b_{21} W_{i}+e_{2 i}
\end{aligned}
$$


$Y_{j i}$ is the measure of the outcome $Y$ for participant $i$ in instance $j$. The measurement of participant $i$ on the between-participant variable is denoted $W_{i}$. Notice that this measurement does not have a subscript $j$ because it is not measured repeatedly, but, rather, is assumed to be constant across instances. The $e_{j i}$ 's are the errors in estimation for participant $i$ in instance $j$, and are assumed to be normally distributed with mean zero and variance $\sigma_{j}^{2}$ and correlation $\rho$ for observations from the same participant and correlation of 0 for observations from different participants. In this model, the relationship between $W$ and $Y$ is allowed to differ by instance (i.e., each of the $b_{j 1}$ 's are allowed to differ), reflecting an interaction between $W$ and instance. The coefficient $b_{11}$ represents the relationship between $W$ and $Y$ in the first instance. The coefficient $b_{21}$ represents the relationship between $W$ and $Y$ in the second instance. When $b_{11} \neq b_{21}$ the relationship between $W$ and $Y$ depends on instance (i.e., there is an interaction between instance and $W$ ). But if $b_{11}$ is equal to $b_{21}$ then there is no interaction and the relationship between $W$ and $Y$ is constant across instances.

To test a moderation hypothesis, a test of whether $b_{11}=b_{21}$ is needed. When these two estimates are in separate models, it is difficult to test this hypothesis using regression. However, if one of these equations is subtracted from the other, a regression coefficient which reflects the difference between $b_{11}$ and $b_{21}$ is included in the resulting equation.:

$$
\begin{aligned}
Y_{2 i}-Y_{1 i} & =b_{20}-b_{10}+\left(b_{21}-b_{11}\right) W_{i}+\left(e_{2 i}-e_{1 i}\right) \\
Y_{D_{i}} & =b_{0}+b_{1} W_{i}+e_{i}
\end{aligned}
$$

Regress the difference in $Y, Y_{2 i}-Y_{1 i}=Y_{D_{i}}$, onto $W$. The regression coefficient for $W\left(b_{1}\right.$ in Equation 2.19) is an estimate of the difference between $b_{11}$ and $b_{21}$. If $b_{1}$ 
is zero, then the difference between $b_{11}$ and $b_{21}$ is zero, meaning $b_{11}=b_{21}$ and there is no interaction between $W$ and instance. However, if $b_{1}$ does not equal zero, this implies that $b_{11}$ and $b_{21}$ are not equal, which means the relationship between $W$ and $Y$ depends on the instance. This matches the intuitive understanding of an interaction as a difference in slopes. Judd et al. (1996) explain that a hypothesis test on an estimate of $b_{1}$ provides a test of interaction in the two-instance repeated-measures design.

Support for the claim that the relationship between instance and $Y$ depends on $W$ is the equivalent of saying the relationship between $W$ and $Y$ depends on instance. In the two-instance repeated-measures case, the relationship between instance and the outcome variable can be expressed as the predicted change in the outcome from one instance to the other, reflected in $Y_{2}-Y_{1}$. Moderation of the relationship between instance and $Y$ would mean that this difference depends on some other variable (e.g., $W)$. This would mean that $W$ predicts the difference between $Y_{1}$ and $Y_{2}$. Equation 2.19 is a model for just this, where the degree to which the two outcomes are expected to differ is a linear function of $W$, and the degree to which this difference depends on $W$ is expressed in the regression coefficient represented by $b_{1}=b_{21}-b_{11}$ This means that if $b_{21}-b_{11} \neq 0$ then the difference between instance on the outcome variable depends on $W$. This would mean that $W$ moderates the relationship between instance and the outcome. Since $b_{1}$ is also a measure of how much instance moderates the relationship between $W$ and $Y$, the symmetry argument holds in the repeatedmeasures case. 


\subsubsection{Estimation and Hypothesis Testing}

To estimate the regression coefficients in Equation 2.19, you can use linear regression analysis to predict the differences in the outcome variable from the betweenparticipant variable $W$ :

$$
\widehat{Y_{D_{i}}}=\hat{b}_{0}+\hat{b}_{1} W_{i}
$$

Here, $\hat{b}_{1}$ is an estimate of the degree to which $W$ moderates the effect of instance on the outcome $Y$. Conducting a hypothesis test on $\hat{b}_{1}$ is a test of interaction between $W$ and instance. If $\hat{b}_{1}$ is significantly different from zero, the null hypothesis that that $b_{11}-b_{21}=0$ is rejected, meaning that the relationship between instance and $Y$ depends on $W$.

Information about the interaction is very useful, but we may have additional questions. Is effect of instance present for those high on $W$, low on $W$, average on $W$ ? At what point along the moderator would the effect of instance be zero? Negative? Positive? These questions can be answered by probing the interaction.

\subsubsection{Probing}

Just as in between-participant designs, the simple-slopes and Johnson-Neyman procedures can be used to probe moderation effects in two-instance repeated-measures designs, though they have only recently been described in this context before (Montoya, in press). I describe how to probe the effect of instance on the outcome variable at different values of $W$ using both the simple-slopes method and Johnson-Neyman procedure where they apply. 


\section{Probing effect of instance on the outcome}

We may be interested in estimating and conducting inference on the effect of instance at specific values of the moderator $W$. A test of interaction examines if the difference in the outcomes depends on the moderator. However, a test of interaction does not provide an estimate of the effect of instance for the group of individuals with a specific score on the moderator. It could be that people high on $W$ would show little difference on the outcomes. On the other hand, it could be that people with low $W$ show a large difference on the outcomes. This type of hypothesis can be tested by estimating the effect of instance at specific values of the moderator using the simple-slopes method. Alternatively, regions of significance can be defined using the Johnson-Neyman method. This analysis would show where along the betweenparticipant variable there is a significant effect of instance on the outcome and where this effect is not significant.

\section{Simple slopes}

The simple-slopes method relies on choosing a point on the moderator $W$, say $w$ then estimating the effect of instance on the outcome at the specific value $W=w$. Using Equation 2.20, an estimate of the effect of instance on the outcome at a specific

value of $W$ is $\widehat{\theta_{X \rightarrow Y}}(W=w)=\hat{b}_{0}+\hat{b}_{1} w$, where $X$ denotes instance and $\widehat{\theta_{X \rightarrow Y}}(W=w)$ denotes the estimated effect of instance on the outcome variable $Y$ as a function of $W$. This is the estimate of the difference in the outcome variables between instances at a specific value of $W$. The variance of $\widehat{\theta_{X \rightarrow Y}}(W=w)$ can be estimated as

$$
\widehat{\operatorname{var}}\left(\widehat{\theta_{X \rightarrow Y}}(W=w)\right)=\widehat{\operatorname{var}}\left(\hat{b}_{0}\right)+w^{2} \widehat{\operatorname{var}}\left(\hat{b}_{1}\right)+2 w \widehat{\operatorname{cov}}\left(\hat{b}_{0}, \hat{b}_{1}\right)
$$


The estimated variance of $\widehat{\theta_{X \rightarrow Y}}(W=w)$ is a function of the chosen value of the moderator $w$, the estimated variance of $\hat{b}_{0}\left(\widehat{\operatorname{var}}\left(\hat{b}_{0}\right)\right)$, the estimated variance of $\hat{b}_{1}\left(\widehat{\operatorname{var}}\left(\hat{b}_{1}\right)\right)$, and the estimated covariance between $\hat{b}_{0}$ and $\hat{b}_{1}\left(\widehat{\operatorname{cov}}\left(\hat{b}_{0}, \hat{b}_{1}\right)\right)$. The estimates of the variances and covariances of the regression coefficients are available through most statistical packages that estimate regression models. However, programs used to conduct regression analyses typically don't calculate $\widehat{\theta_{X \rightarrow Y}}(W=w)$ or $\widehat{\operatorname{var}}\left(\widehat{\theta_{X \rightarrow Y}}(W=w)\right)$ without additional assistance.

The ratio of the estimate of $\theta_{X \rightarrow Y}(W)$ to its standard error is $t$-distributed with $N-q-1$ degrees of freedom, where $N$ is the number of observations and $q$ is the number of predictors in the regression model. In the case of Equation 2.20, $q=1$. Specific values of $W$ can be plugged into the equation for $\widehat{\theta_{X \rightarrow Y}}(W)$ and $\widehat{\operatorname{var}}\left(\widehat{\theta_{X \rightarrow Y}}(W)\right)$. The ratio $\left.\widehat{\theta_{X \rightarrow Y}}(W)\right) / \sqrt{\widehat{\operatorname{var}}\left(\widehat{\theta_{X \rightarrow Y}}(W)\right)}$ can be calculated and compared to a critical $t$ statistic with the appropriate degrees of freedom. Alternatively, a $p$-value can be calculated from the calculated $t$-statistic.

\section{Johnson-Neyman Procedure}

Just as in moderation in between subjects designs, the equation for the ratio of $\widehat{\theta_{X \rightarrow Y}}(W)$ to its standard error can be used to calculate the point(s) along the range of $W$ where the ratio is exactly equal to the critical $t$-value that correspond to a selected $\alpha$ level. These points mark the boundaries of significance for the relationship between instance and the outcome. By solving for these points, the Johnson-Neyman technique defines the pattern of significance for the relationship between instance and the outcome across the entire range of $W$.

By setting the absolute value of the ratio of $\widehat{\theta_{X \rightarrow Y}}(W)$ to its standard error equal to value of the critical $t$-value, and solving for $W$, these points can be found. The 
critical $t$-value is denoted as $t_{\alpha / 2, d f}^{*}$. Ratios greater than $t_{\alpha / 2, d f}^{*}$ are significant at level $\alpha$.

$$
\begin{aligned}
& t_{\alpha / 2, d f}^{*}=\left|\frac{\widehat{\theta}_{X \rightarrow Y}(W)}{\sqrt{\widehat{\operatorname{var}}\left(\widehat{\theta_{X \rightarrow Y}}(W)\right)}}\right| \\
& t_{\alpha / 2, d f}^{*}=\left|\frac{\hat{b}_{0}+\hat{b}_{1} W}{\sqrt{\widehat{\operatorname{var}}\left(\hat{b}_{0}\right)+W^{2} \widehat{\operatorname{var}}\left(\hat{b}_{1}\right)+2 W \widehat{\operatorname{cov}}\left(\hat{b}_{0}, \hat{b}_{1}\right)}}\right| \\
& t_{\alpha / 2, d f}^{*^{2}}=\frac{\left(\hat{b}_{0}+\hat{b}_{1} W\right)^{2}}{\widehat{\operatorname{var}}\left(\hat{b}_{0}\right)+W^{2} \widehat{\operatorname{var}}\left(\hat{b}_{1}\right)+2 W \widehat{\operatorname{cov}}\left(\hat{b}_{0}, \hat{b}_{1}\right)} \\
& 0=\left(\hat{b}_{0}^{2}-t_{\alpha / 2, d f}^{*^{2}} \widehat{\operatorname{var}}\left(\hat{b}_{0}\right)+\left(2 \hat{b}_{1} \hat{b}_{0}-2 t_{\alpha / 2, d f}^{*^{2}} \widehat{\operatorname{cov}}\left(\hat{b}_{0}, \hat{b}_{1}\right) W+\left(\hat{b}_{1}^{2}-t_{\alpha / 2, d f}^{*^{2}} \widehat{\operatorname{var}}\left(\hat{b}_{1}\right)\right) W^{2}\right.\right. \\
& W=\frac{-\left(2 \hat{b}_{1} \hat{b}_{0}-2 t_{\alpha / 2, d f}^{*^{2}} \widehat{\operatorname{cov}}\left(\hat{b}_{0}, \hat{b}_{1}\right)\right)}{2\left(\hat{b}_{1}^{2}-t_{\alpha / 2, d f}^{*^{2}} \widehat{\operatorname{var}}\left(\hat{b}_{1}\right)\right.} \\
& \pm \frac{\sqrt{\left(2 \hat{b}_{1} \hat{b}_{0}-2 t_{\alpha / 2, d f}^{*^{2}} \widehat{\operatorname{cov}}\left(\hat{b}_{0}, \hat{b}_{1}\right)\right)^{2}-4\left(\hat{b}_{0}^{2}-t_{\alpha / 2, d f}^{*^{2}} \widehat{\operatorname{var}}\left(\hat{b}_{0}\right)\right)\left(\hat{b}_{0}^{2}-t_{\alpha / 2, d f}^{*^{2}} \widehat{\operatorname{var}}\left(\hat{b}_{1}\right)\right)}}{2\left(\hat{b}_{1}^{2}-t_{\alpha / 2, d f}^{*^{2}} \widehat{\operatorname{var}}\left(\hat{b}_{1}\right)\right.}
\end{aligned}
$$

In its mathematical form, there are always two solutions to this problem. However, these two solutions are not always interpretable. Just as in the between subjects case, solutions can be imaginary or fall outside of the range of the observed data, neither of which should be interpreted. Even when transition points are found within the range of the data, it is important to note how much of the data is above or below these points in order to determine how much to trust them. Without data surrounding the Johnson-Neyman points, there is no evidence that the observed trend continues outside of the range of the observed data, and thus these points are neither meaningful nor interpretable. 


\subsection{Summary}

This chapter has outlined previous work on mediation in two-instance repeatedmeasures designs, emphasizing how this approach has evolved from the piecewise approach of Judd et al. (2001) to a path-analytic approach as proposed by Montoya and Hayes (2017). Additionally, I described moderation in two-instance repeated measures designs, including how to test an interaction between the repeated-measures factor and a between-subjects moderator. The primary contribution of this dissertation is showing how these two approaches can be combined to allow indirect effects to be moderated, how to estimate conditional indirect effects, and test if mediation is moderated. In the next chapter, I describe how the approaches discussed in this chapter can be combined to assess conditional process models in two-instance repeated-measures designs. 


\section{Chapter 3: Conditional Process Analysis in Two-Instance Repeated-Measures Designs}

In this chapter, I provide a general conditional process model for the two-instance repeated measures design. This model has one moderator and one mediator. I will describe how simplifications of this general model correspond to more commonly used conditional process models, such as first-stage conditional process models and second-stage conditional process models. For these models, I will derive the index of moderated mediation and describe how to test moderated mediation.

\subsection{A General Conditional Process Model}

In the most general model with a single mediator $M$ and a single moderator $W$, the moderator is allowed to moderate three paths: the path from $X$ to $M$, the path from $M$ to $Y$, and the direct path from $X$ to $Y$. First, I describe how to allow the relationship between instance (the repeated-measures factor) and the mediator $M$ to differ by $W$. This is identical to the case of moderation in a two-instance repeated measures design, where now the outcome is the mediator rather than the $Y$ variable.

Writing out the model for each mediator in each instance, I allow the moderator $W$ to be a predictor of the mediator in each instance. When the difference between these two models is taken, it is clear that the coefficient for $W$ is an estimate of how 
much the effect of instance changes as $W$ changes.

$$
\begin{aligned}
M_{1 i} & =a_{10}+a_{11} W_{i}+e_{M_{1 i}} \\
M_{2 i} & =a_{20}+a_{21} W_{i}+e_{M_{2 i}} \\
M_{2 i}-M_{1 i} & =a_{20}-a_{10}+\left(a_{21}-a_{11}\right) W_{i}+e_{M_{2 i}}-e_{M_{1 i}} \\
M_{2 i}-M_{1 i} & =a_{0}+a_{1} W_{i}+e_{M_{i}}
\end{aligned}
$$

In Equations 3.1 and 3.2, the relationship between $W$ and $M$ is allowed to differ by instance, thus allowing the relationship between instance and $M$ to differ by $W$. The difference between $M_{2}$ and $M_{1}$ is an estimate of the effect of instance on the mediator. When $a_{1}$ is different from zero, $W$ predicts the effect of instance on the mediator, meaning this effect depends on the level of $W$. When $a_{1}$ is zero, this means that the effect of instance on the mediator is constant across the range of the moderator (i.e., no moderation).

In the next models, I allow the relationships between $M$ and $Y$ as well as instance and $Y$ to differ by $W$. I begin with the original equations from the mediation models but adding $W$ as a predictor:

$$
\begin{aligned}
Y_{1 i} & =c_{1}^{* *}+b_{11} M_{1 i}+b_{12} W_{i}+e_{Y_{1 i}} \\
Y_{2 i} & =c_{2}^{* *}+b_{21} M_{2 i}+b_{22} W_{i}+e_{Y_{2 i}} \\
Y_{2 i}-Y_{1 i} & =\left(c_{2}^{\prime *}-c_{1}^{\prime *}\right)+b_{21} M_{2 i}-b_{11} M_{1 i}+\left(b_{22}-b_{12}\right) W_{i}+\left(e_{Y_{2 i}}-e_{Y_{1 i}}\right)
\end{aligned}
$$

In Equation 3.7, if the relationship between $W$ and $Y$ is constant across instances (i.e., $b_{12}=b_{22}$ ) then the coefficient for $W$ in Equation 3.7 will be zero. This means that controlling for the two mediators, $W$ does not predict the difference in $Y$; that is, 
$W$ does not predict the effect of instance on $Y$ after controlling for the mediator. By allowing $W$ to be a predictor in the equation for each outcome with different weights, $W$ is allowed to moderate the direct effect of instance on $Y$. Thus, in Equation 3.7, the direct effect is allowed to be moderated by $W$ by allowing $W$ to be a predictor in the difference of $Y$ while controlling for the mediators.

To allow the relationship between $M$ and $Y$ to depend on $W$, let the effect of $M$ on $Y$ conditional on instance be a linear function of the moderator. First consider a simple model which constrains the degree to which the relationship between $M$ and $Y$ depends on $W$ to be constant across instance. $W$ is still a predictor on its own, which allows for the direct effect to be moderated.

$$
\begin{aligned}
& Y_{1 i}=c_{1}^{\prime *}+\left(b_{11}+b_{.3} W_{i}\right) M_{1 i}+b_{12} W_{i}+e_{Y_{1 i}} \\
& Y_{2 i}=c_{2}^{\prime *}+\left(b_{21}+b_{.3} W_{i}\right) M_{2 i}+b_{22} W_{i}+e_{Y_{2 i}}
\end{aligned}
$$

Here I've allowed the relationship between $M$ and $Y$ when $W$ is zero to differ by instances (i.e., $b_{11}$ is not constrained to be equal to $b_{21}$ ). However, the degree to which the relationship between $M$ and $Y$ changes as $W$ changes is constrained across conditions to be $b .3$. This means that the degree to which $W$ moderates the relationship between $M$ and $Y$ is not moderated by instance.

Next, by taking the difference between the two equations and grouping like terms, the result is

$$
\begin{aligned}
Y_{2 i}-Y_{1 i}= & \left(c_{2}^{*}-c_{1}^{\prime *}\right)+\left(b_{21}+b_{.3} W_{i}\right) M_{2 i}-\left(b_{11}+b_{.3} W_{i}\right) M_{1 i}+\left(b_{22}-b_{12}\right) W_{i} \\
& +e_{Y_{2 i}}-e_{Y_{1 i}} \\
= & \left(c_{2}^{\prime *}-c_{1}^{\prime *}\right)+b_{21} M_{2 i}-b_{11} M_{1 i}+b_{.3} W_{i}\left(M_{2 i}-M_{1 i}\right)+\left(b_{22}-b_{12}\right) W_{i} \\
& +e_{Y_{2 i}}-e_{Y_{1 i}}
\end{aligned}
$$


Using the same rotation applied in the mediation model proposed by Judd et al. (2001), but using the average instead of the sum of the mediators, the result is

$$
\begin{aligned}
Y_{2 i}-Y_{1 i}= & \left(c_{2}^{*}-c_{1}^{\prime *}\right)+\frac{b_{21}+b_{11}}{2}\left(M_{2 i}-M_{1 i}\right)+\left(b_{21}-b_{11}\right) \frac{M_{2 i}+M_{1 i}}{2} \\
& +b_{.3} W_{i}\left(M_{2 i}-M_{1 i}\right)+\left(b_{22}-b_{12}\right) W_{i}+e_{Y_{2 i}}-e_{Y_{1 i}}
\end{aligned}
$$

When predicting the difference between the outcome variables, including the difference between the mediators $\left(M_{2 i}-M_{1 i}\right)$, the average of the mediators $\frac{M_{2 i}+M_{1 i}}{2}$, the moderator $W$, and the product of the moderator and the difference between the mediators $W_{i}\left(M_{2 i}-M_{1 i}\right)$ allows the $M$ to $Y$ relationship to be moderated by $W$. The coefficient for the difference between the mediators, $\frac{b_{21}+b_{11}}{2}$, is the effect of $M$ on $Y$ when $W$ is zero, averaged across instance. The coefficient for the average of the mediators, $b_{21}-b_{11}$, is the difference between the effect of $M$ on $Y$ when $W=0$. The coefficient for the product of the moderator and the difference in the mediators, $b_{.3}$, is the degree to which the relationship between $M$ and $Y$ changes as $W$ changes (in both instances). This coefficient, when it is different from zero reflects moderation of the relationship between $M$ and $Y$ by $W$. As before, the coefficient for $W, b_{22}-b_{21}$, reflects the degree to which the direct effect is moderated.

Constraining the degree to which changes in $W$ influence the relationship between $M$ and $Y$ across instances at $b_{.3}$ may seem overly restrictive, as it does not allow for the interaction between $M$ and $W$ to be uniquely estimated in each instance. Next, I will consider a model where the moderation of the effect of $M$ and $Y$ is allowed to differ by instance. This is equivalent to allowing a three-way interaction between $M$, $W$, and instance. This is the most general model I will describe. 
I begin with the same model as above, but this time allowing the coefficient for the product between $M$ and $W$ to differ by instance.

$$
\begin{aligned}
& Y_{1 i}=c_{1}^{\prime *}+\left(b_{11}+b_{13} W_{i}\right) M_{1 i}+b_{12} W_{i}+e_{Y_{1 i}} \\
& Y_{2 i}=c_{2}^{\prime *}+\left(b_{21}+b_{23} W_{i}\right) M_{2 i}+b_{22} W_{i}+e_{Y_{2 i}}
\end{aligned}
$$

Now both the relationship between $M$ and $Y$ when $W$ is zero is allows to differ by instance, and the degree to which the relationship between $M$ and $Y$ changes as $W$ changes is allowed to differ by instance. Described another way, this means that the degree to which $W$ moderates the relationship between $M$ and $Y$ is allowed to be moderated by instance.

Next, taking the difference between the two equations, grouping like terms, and applying the rotation from Judd et al. (2001) using the average instead of the sum results in

$$
\begin{aligned}
Y_{2 i}-Y_{1 i}= & \left(c_{2}^{\prime *}-c_{1}^{\prime *}\right)+\left(b_{21}+b_{23} W_{i}\right) M_{2 i}-\left(b_{11}+b_{13} W_{i}\right) M_{1 i}+\left(b_{22}-b_{12}\right) W_{i} \\
& +e_{Y_{2 i}}-e_{Y_{1 i}} \\
= & \left(c_{2}^{\prime *}-c_{1}^{\prime *}\right)+\frac{b_{21}+b_{23} W_{i}+b_{11}+b_{13} W_{i}}{2}\left(M_{2 i}-M_{1 i}\right) \\
& +\left(b_{21}+b_{23} W_{i}-b_{11}-b_{13} W_{i}\right) \frac{M_{2 i}+M_{1 i}}{2}+\left(b_{22}-b_{12}\right) W_{i}+e_{Y_{2 i}}-e_{Y_{1 i}} \\
= & \left(c_{2}^{\prime *}-c_{1}^{\prime *}\right)+\frac{b_{21}+b_{11}}{2}\left(M_{2 i}-M_{1 i}\right)+\frac{b_{23}+b_{13}}{2} W_{i}\left(M_{2 i}-M_{1 i}\right) \\
& +\left(b_{21}-b_{11}\right) \frac{M_{2 i}+M_{1 i}}{2}+\left(b_{23}-b_{13}\right) W_{i} \frac{M_{2 i}+M_{1 i}}{2}+\left(b_{22}-b_{12}\right) W_{i} \\
& +e_{Y_{2 i}}-e_{Y_{1 i}}
\end{aligned}
$$

This equation is quite similar to Equation 3.10, where we are predicting the difference between the outcome variables using the difference between the mediators, the 
average of the mediators, the moderator, and the product of the moderator and the difference between the mediators. But there is an additional term in this equation which is the product of the moderator and the average of the mediators. The coefficient for this term $\left(b_{23}-b_{13}\right)$ is the difference in the degree to which $W$ moderates the relationship between $M$ and $Y$ in each instance. This tells us if the moderation of the $M-Y$ relationship by $W$ is moderated by instance. The interpretation of all other parameters stays the same with one exception. Previously the coefficient for the product of the moderator and the difference of the mediators estimated the degree to which the relationship between $M$ and $Y$ changes as $W$ changes (in both instances). Now the coefficient is an estimate of the degree to which the relationship between $M$ and $Y$ changes as $W$ changes, averaged over instance.

Just as in the case of mediation, grand mean centering the average term will aid interpretation of the direct effect. I do this by adding in and subtracting out $\left(b_{21}-b_{11}+\left(b_{23}-b_{13}\right) W_{i}\right)^{\frac{\overline{M_{2} \cdot+M_{1}}}{2}}$.

$$
\begin{aligned}
Y_{2 i}-Y_{1 i}= & \left(c_{2}^{\prime *}-c_{1}^{\prime *}\right)+\left(b_{21}-b_{11}+\left(b_{23}-b_{13}\right) W_{i}\right) \frac{\overline{M_{2 \cdot}+M_{1} .}}{2} \\
& +\frac{b_{21}+b_{11}}{2}\left(M_{2 i}-M_{1 i}\right)+\frac{b_{23}+b_{13}}{2} W_{i}\left(M_{2 i}-M_{1 i}\right) \\
& +\left(b_{21}-b_{11}\right)\left(\frac{M_{2 i}+M_{1 i}}{2}-\frac{\left.\frac{2}{M_{2 .}+M_{1 .}}\right)}{2}\right) \\
& +\left(b_{23}-b_{13}\right) W_{i}\left(\frac{M_{2 i}+M_{1 i}}{2}-\frac{\frac{2}{M_{2 \cdot}+M_{1 \cdot}}}{2}\right)+\left(b_{22}-b_{12}\right) W_{i} \\
& +e_{Y_{2 i}}-e_{Y_{1 i}}
\end{aligned}
$$

Grouping terms and simplifying notation, I rewrite Equation 3.14 as 


$$
\begin{aligned}
Y_{2 i}-Y_{1 i}= & c^{\prime}+b_{1}\left(M_{2 i}-M_{1 i}\right)+b_{2}\left(\frac{M_{2 i}+M_{1 i}}{2}-\frac{\overline{M_{2 \cdot}+M_{1 \cdot}}}{2}\right)+b_{3} W_{i} \\
& +b_{4} W_{i}\left(M_{2 i}-M_{1 i}\right)+b_{5} W_{i}\left(\frac{M_{2 i}+M_{1 i}}{2}-\frac{\overline{M_{2 \cdot}+M_{1 \cdot}}}{2}\right)+e_{Y_{i}}
\end{aligned}
$$

where

$$
\begin{aligned}
c^{\prime} & =c_{2}^{\prime *}-c_{1}^{\prime *}+b_{2} \frac{\overline{M_{2 \cdot}+M_{1}}}{2} \\
b_{1} & =\frac{b_{21}+b_{11}}{2} \\
b_{2} & =b_{21}-b_{11} \\
b_{3} & =b_{22}-b_{12}+\left(b_{23}-b_{13}\right) \frac{\overline{M_{2 \cdot}+M_{1}}}{2} \\
b_{4} & =\frac{b_{23}+b_{13}}{2} \\
b_{5} & =\left(b_{23}-b_{13}\right) \\
e_{Y_{i}} & =e_{Y_{2 i}}-e_{Y_{1 i}}
\end{aligned}
$$

In combination Equation 3.4 and 3.15 represent the most general model which allows $W$ to moderate the effect of instance on the mediator, the effect of the mediator on the outcome, and the direct effect of instance on the outcome. Additionally, the degree to which $W$ moderates the relationship between $M$ and $Y$ is also moderated by instance.

\subsubsection{Defining Conditional Effects}

Using Equations 3.4 and 3.15, we can define the important conditional effects for assessing mediation and moderation of mediation.

Equation 3.4 gives the estimate of the conditional effect of instance on the mediator:

$$
\theta_{X \rightarrow M}(W)=a_{0}+a_{1} W
$$


This is how much we would expect the mediators in each instance to differ given a specific value of the moderator, $W$. If we take the terms in Equation 3.15 and group terms based on the difference and mean centered average of the mediators we get

$$
Y_{2 i}-Y_{1 i}=c^{\prime}+\left(b_{1}+b_{4} W_{i}\right)\left(M_{2 i}-M_{1 i}\right)+\left(b_{2}+b_{5} W_{i}\right)\left(\frac{M_{2 i}+M_{1 i}}{2}-\frac{\overline{M_{2 .}+M_{1 \cdot}}}{2}\right)+b_{3} W_{i}+e_{Y_{i}}
$$

The coefficient of the difference of the mediators is $b_{1}+b_{4} W_{i}$ which estimates the conditional effect of $M$ on $Y$. If it is not clear that this function represents the relationship between $M$ and $Y$, remember that $b_{1}$ and $b_{4}$ are combinations of the effect of $M$ on $Y$ in each instance.

$$
b_{1}+b_{4} W=\frac{b_{21}+b_{11}}{2}+\frac{b_{23}+b_{13}}{2} W
$$

Rearranging terms shows that $b_{1}+b_{4} W$ is the conditional effect of $M$ on $Y$, averaged across instance.

$$
\begin{aligned}
b_{1}+b_{4} W & =\frac{\left(b_{21}+b_{23} W\right)+\left(b_{11}+b_{13} W\right)}{2} \\
& =\frac{\theta_{M \rightarrow Y}(W \mid \text { Instance } 2)+\theta_{M \rightarrow Y}(W \mid \text { Instance } 1)}{2}
\end{aligned}
$$

Thus the conditional effect of $M$ on $Y$ (averaged over instance) is defined as

$$
\theta_{M \rightarrow Y}(W)=b_{1}+b_{4} W
$$

The conditional direct effect is the expected difference in outcomes for an individual who shows no difference on the mediators (thus any expected difference in the 
outcomes cannot be attributed to an indirect effect of instance on $Y$ through $M$ ). By taking Equation 3.15 and conditioning on $M_{2 i}-M_{1 i}=0$ the following equation results

$$
\begin{aligned}
\mathbb{E}\left(Y_{2 i}-Y_{1 i} \mid M_{2 i}-M_{1 i}=0\right)= & c^{\prime}+b_{2}\left(\frac{M_{2 i}+M_{1 i}}{2}-\frac{\overline{M_{2 .}+M_{1 \cdot}}}{2}\right)+b_{3} W_{i} \\
& +b_{5} W_{i}\left(\frac{M_{2 i}+M_{1 i}}{2}-\frac{\overline{M_{2 .}+M_{1 \cdot}}}{2}\right)
\end{aligned}
$$

The direct effect depends both on the average of the mediators and moderator $W$. We can condition on the average of the mediators being at the sample average, thus estimating the direct effect conditional on the average of the mediators being at the sample mean:

$$
\theta_{X \rightarrow Y}\left(\frac{M_{2 i}+M_{1 i}}{2}=\frac{\overline{M_{2 \cdot}+M_{1}}}{2}, W\right)=c^{\prime}+b_{3} W
$$

Thus, for someone who is average on the mediators, the direct effect of instance on the outcome is a function of $W$. Indeed for a one unit increase in $W$ there is expected to be a $b_{3}$ unit increase in the direct effect. Note too that the Equation for $b_{3}, 3.19$, includes a term which involves the sample mean of the average of the mediators, $\frac{\overline{M_{2}+M_{1}}}{2}$. So centering the average of the mediators impacts the estimate of $b_{3}$, illustrating how $b_{3}$ is conditional on the sample mean of the average of the mediators.

The indirect effect, which is conditional on $W$, is of primary interest when considering hypotheses of moderated mediation. The conditional indirect effect is the product of the effect of instance on the mediator and the effect of the mediator on 
the outcome. In the most general model, each of these effects are linear functions of $W$. As such, the indirect effect will be conditional on $W$, or at least allowed to be.

$$
\begin{aligned}
\theta_{X \rightarrow M}(W) \theta_{M \rightarrow Y}(W) & =\left(a_{0}+a_{1} W\right)\left(b_{1}+b_{4} W\right) \\
& =a_{0} b_{1}+\left(a_{0} b_{4}+a_{1} b_{1}\right) W+a_{1} b_{4} W^{2}
\end{aligned}
$$

So unlike many of the other conditional effects in this model, the conditional indirect is not a linear function of $W$. Rather it is a quadratic function of $W$. When estimating this model, specific values of $W$ may be selected and the conditional indirect effect can be estimated (Hayes, 2018a; Edwards \& Lambert, 2007).

Allowing each path in the mediation pathway to be moderated is very general, but it lacks parsimony. If we only expect that certain paths are moderated, then we may want to estimate more parsimonious models. Using the general model proposed above, I now show how to constrain the model, yielding a few different conditional process models each with their own constraints.

\subsection{First Stage Conditional Process Models}

In a first stage conditional process model, $W$ is allowed to moderate the relationship between instance and the mediator. It is not allowed to moderate the relationship between the mediator and the outcome. I will discuss cases when the moderator is allowed to moderate the direct effect and when the direct effect is not moderated. Because the relationship between instance and the mediator is moderated, the equation for the difference in mediators is the same as Equation 3.4 and the conditional effect of instance on the mediators is still represented by Equation 3.23 as a linear function of $W$. 
The relationship between $M$ and $Y$ is fixed across the moderator. So the equations for the outcome must be simplified. First let us consider the case where the direct effect is allowed to be moderated by $W$. Remember this was the case in Equations 3.5 through 3.7. Applying the rotation to Equation 3.7 and centering the average of the mediators, we get

$$
\begin{aligned}
Y_{2 i}-Y_{1 i}= & \left(c_{2}^{\prime *}-c_{1}^{\prime *}\right)+\frac{b_{21}+b_{11}}{2}\left(M_{2 i}-M_{1 i}\right)+\left(b_{21}-b_{11}\right)\left(\frac{M_{2 i}+M_{1 i}}{2}-\frac{\overline{M_{2 \cdot}+M_{1 \cdot}}}{2}\right) \\
& +\left(b_{22}-b_{12}\right) W_{i}+\left(e_{Y_{2 i}}-e_{Y_{1 i}}\right)
\end{aligned}
$$

which can be notationally simplified to

$$
Y_{2 i}-Y_{1 i}=c^{\prime}+b_{1}\left(M_{2 i}-M_{1 i}\right)+b_{2}\left(\frac{M_{2 i}+M_{1 i}}{2}-\frac{\overline{M_{2 \cdot}+M_{1 \cdot}}}{2}\right)+b_{3} W_{i}+e_{Y_{i}}
$$

The effect of the mediator on the outcome, averaging over instance is:

$$
\theta_{M \rightarrow Y}=\left(b_{21}+b_{11}\right) / 2=b_{1}
$$

By constraining $b_{4}$ and $b_{5}$ in the general model (Equation 3.15) to be zero, we get the first-stage conditional process model, which allows the direct effect to be moderated.

If in addition, we did not want the direct effect to be moderated, we can set $b_{3}=0$, which results in the following model for the difference in $Y$ 's:

$$
Y_{2 i}-Y_{1 i}=c^{\prime}+b_{1}\left(M_{2 i}-M_{1 i}\right)+b_{2}\left(\frac{M_{2 i}+M_{1 i}}{2}-\frac{\overline{M_{2 \cdot}+M_{1 \cdot}}}{2}\right)
$$

Now $W$ no longer needs to be included in the model of the outcome. This is the same model as Montoya and Hayes (2017) for the difference in the outcomes in 
the two-instance repeated-measures mediation analysis (Equation 2.9). Though the model for the outcomes is the same as in simple mediation, the model for the mediators is different. The effect of instance on the mediators is allowed to be moderated by $W$, and so the product of this conditional effect of instance on the mediator and the unconditional effect of the mediator on the outcome will still result in a conditional indirect effect.

$$
\theta_{X \rightarrow M}(W) \theta_{M \rightarrow Y}=\left(a_{0}+a_{1} W\right) b_{1}=a_{0} b_{1}+a_{1} b_{1} W
$$

Now the conditional indirect effect is a linear function of $W$, and a test on $a_{1} b_{1}$ will provide a test of whether the indirect effect is moderated by $W$. Here $a_{1} b_{1}$ is called the index of moderated mediation (Hayes, 2015). Note that the coefficient used to represent the effect of the mediators on the outcome does not depend on whether or not the direct effect is allowed to be moderated. It is always $b_{1}$. The definition of the conditional indirect effect will not be changed by whether the direct effect is allowed to be moderated. But whether or not the direct effect is allowed to be moderated will impact the estimate of $b_{1}$, so it is still an important choice to make.

If instead we believed the relationship between the mediator and the outcome was moderated by $W$, we would use a different model with different constraints on the general model. This has been called a second-stage conditional process model, because the second stage of the mediation process is moderated but the first stage is not. 


\subsection{Second Stage Conditional Process Models}

In second stage conditional process models, the moderator does not moderate the relationship between instance and the mediator, but it does moderate the relationship between the mediator and the outcome. Like a first stage conditional process model, the direct effect can be moderated or not; the choice does not impact the conditional indirect effect. Instead of using Equation 3.4 for the model of the mediators, we fix the relationship between $W$ and the mediator to be the same across instance, thus fixing the effect of instance on the mediator across levels of the moderator.

$$
\begin{aligned}
& M_{1 i}=a_{10}+a_{\cdot 1} W_{i}+e_{M_{1 i}} \\
& M_{2 i}=a_{20}+a_{\cdot 1} W_{i}+e_{M_{2 i}}
\end{aligned}
$$

When the difference between these two equations is taken, the $W$ terms disappear, and $W$ is not needed in the model of difference of the mediators.

$$
\begin{aligned}
M_{2 i}-M_{1 i} & =a_{20}-a_{10}+\left(a_{\cdot 1}-a_{\cdot 1}\right) W_{i}+e_{M_{2 i}}-e_{M_{1 i}} \\
M_{2 i}-M_{1 i} & =a_{0}+e_{M_{i}} \\
\theta_{X \rightarrow M} & =a_{0}
\end{aligned}
$$

Note this model is the same model which was used for the difference in the mediators when doing simple mediation analysis (Equation 2.6). Alternatively, this would be like using Equation 3.4 but constraining $a_{1}=0$. Even though the relationship between instance and the mediator is not conditioned on $W$, the relationship between the mediator and the outcome will be allowed to differ depending on $W$, so 
the indirect effect will still be conditional. I first consider the case where both the relationship between the mediator and the outcome and the direct effect are moderated. This is represented by Equation 3.15. Thus, the conditional effect of $M$ on $Y$ is $\theta_{M \rightarrow Y}(W)=b_{1}+b_{4} W$. If we do not want the direct effect to be moderated, $b_{3}$ can be constrained to 0 , meaning the $W$ would not be included in the model on its own, but only in the product with the difference in the mediators and the average.

$$
\begin{aligned}
Y_{2 i}-Y_{1 i}= & c^{\prime}+b_{1}\left(M_{2 i}-M_{1 i}\right)+b_{2}\left(\frac{M_{2 i}+M_{1 i}}{2}-\frac{\overline{M_{2 \cdot}+M_{1 \cdot}}}{2}\right) \\
& +b_{4} W_{i}\left(M_{2 i}-M_{1 i}\right)+b_{5} W_{i}\left(\frac{M_{2 i}+M_{1 i}}{2}-\frac{\overline{M_{2 .}+M_{1 \cdot}}}{2}\right)+e_{Y_{i}}
\end{aligned}
$$

The relationship between the mediator and the outcome does not depend on whether the direct effect is moderated. Estimating the conditional indirect effect will proceed similarly, regardless of whether the direct effect is moderated. However, including $W$ in the model will impact the estimates of $b_{1}$ and $b_{4}$ and thus the estimates of these coefficients will depend on whether or not $W$ is included in the model. Care should be taken to consider whether theory predicts a moderated direct effect or not, and to include $W$ in the model in the appropriate places. The product of the effect of instance on the mediator and the conditional effect of the mediator on the outcome is the conditional indirect effect.

$$
\theta_{X \rightarrow M} \theta_{M \rightarrow Y}(W)=a_{0}\left(b_{1}+b_{4} W\right)=a_{0} b_{1}+a_{0} b_{4} W
$$

Again the conditional indirect effect is a linear function of $W$ and a test on $\hat{a}_{0} \hat{b}_{4}$ will provide a test of whether the indirect effect is moderated by $W$, where $a_{0} b_{4}$ is the index of moderated mediation. 


\subsection{Models where Instance is not a Moderator}

Up to this point, I have allowed instance to moderate the relationship between the mediator and the outcome. Judd et al. (2001) discuss the mediation model where this is not the case. In this model, instead of using Equation 2.9 we would use the following equation to estimate the relationship between $M$ and $Y$ :

$$
Y_{2 i}-Y_{1 i}=c^{\prime}+b\left(M_{2 i}-M_{1 i}\right)+e_{Y_{i}^{\prime}}
$$

Note that there is no average of the mediators included in this equation. This is because, if we assume that the relationship between $M_{1}$ and $Y_{1}$ is equal to that of the relationship between $M_{2}$ and $Y_{2}$ then there is no need for the rotation suggested by Judd et al. (2001) when we take the difference between the equations for $Y_{2 i}$ and $Y_{1 i}$.

$$
\begin{aligned}
Y_{1 i} & =c_{1}^{\prime}+b M_{1 i}+e_{Y_{1 i}^{\prime}} \\
Y_{2 i} & =c_{2}^{\prime}+b M_{2 i}+e_{Y_{2 i}^{\prime}} \\
Y_{2 i}-Y_{1 i} & =\left(c_{2}^{\prime}-c_{1}^{\prime}\right)+b\left(M_{2 i}-M_{1 i}\right)+e_{Y_{2 i}^{\prime}}-e_{Y_{1 i}^{\prime}}
\end{aligned}
$$

All previous models described in this chapter allow for the relationship between $M$ and $Y$ to depend on instance. However, if we wanted to restrict the relationships to be the same across instances, the estimating equation for the model of the difference in the outcomes would be the same except that it would exclude any terms which involve the average of the repeated-measurements of the mediators $\left(\frac{M_{1 i}+M_{2 i}}{2}\right)$.

For example, consider the most general model outlined by Equations 3.4 and 3.15. The equation for the difference in the mediators does not change. However, the equation for the difference in the outcomes would change. Consider new versions of 
Equations 3.11 and 3.12 where we fix the relationship between $M$ and $Y$ to be the same across conditions:

$$
\begin{aligned}
& Y_{1 i}=c_{1}^{\prime}+\left(b_{1}+b_{3} W_{i}\right) M_{1 i}+b_{12} W_{i}+e_{Y_{1 i}} \\
& Y_{2 i}=c_{2}^{\prime}+\left(b_{1}+b_{3} W_{i}\right) M_{2 i}+b_{22} W_{i}+e_{Y_{2 i}}
\end{aligned}
$$

When we take the difference between these two equations, the difference between the mediators still emerges as a predictor of the difference in the outcomes, but now the average is not needed.

$$
\begin{aligned}
Y_{2 i}-Y_{1 i} & =c_{2}^{\prime}-c_{1}^{\prime}+\left(b_{1}+b_{3} W_{i}\right)\left(M_{2 i}-M_{1 i}\right)+\left(b_{22}-b_{12}\right) W_{i}+e_{Y_{2 i}}-e_{Y_{1 i}} \\
& =c^{\prime}+b_{1}\left(M_{2 i}-M_{1 i}\right)+b_{2} W_{i}+b_{3} W_{i}\left(M_{2 i}-M_{1 i}\right)+e_{Y_{i}}
\end{aligned}
$$

where

$$
\begin{aligned}
c^{\prime} & =c_{2}^{\prime}-c_{1}^{\prime} \\
b_{2} & =\left(b_{22}-b_{12}\right) \\
e_{Y_{i}} & =e_{Y_{2 i}}-e_{Y_{1 i}}
\end{aligned}
$$

Equation 3.47 is the same as Equation 3.15 if $b_{2}$ and $b_{5}$ were fixed to zero. Similarly, if a researcher wanted to estimate any of the other conditional process models described in Chapter 3, but constrain the relationship between $M$ and $Y$ to be the same across instances, they would just eliminate all terms that involved the average of the repeated-measurements of the mediator. 
There are potential benefits and costs to estimating models where the relationship between $M$ and $Y$ is fixed across instances. Benefits are that so long as this assumption is correct, a more parsimonious model is being estimated. This should result in a slight increase in statistical power; however, there are no simulation results as of now that show this to be true. Interpreting the results of these analyses is less arduous than interpreting results where the relationship between $M$ and $Y$ is allowed to vary across instance. As you'll see in Chapter 4, many of the effects, particularly the direct effect, are interpreted for individuals who are "at the sample mean for the average of the mediators." When we assume that the relationship between $M$ and $Y$ is the same across instances, the interpretation of different parameters is more streamlined.

There are also some potential costs of estimating models where we assume that the relationship between $M$ and $Y$ is the same across instances. First, if this assumption is not true, this may result in bias in the parameters and/or loss of power to detect the indirect effect. Future research should examine these types of costs using simulation studies. Additionally, by allowing the effects to differ across instance, each analysis includes an estimate of this difference and inference can then be used to make a decision about whether or not this seems reasonable. So though the researcher may not believe it to be so, allowing the relationship between $M$ and $Y$ to differ across instances gives the researcher an opportunity to test this belief, rather than just assuming that it is true. However, I would like to point out that I do not recommend the strategy of estimating the model which allows the effect of $M$ on $Y$ to differ by instance, and then if the coefficient for the average of the mediators is not significant, simplifying the model and reestimating. This introduces issues of sequential testing and changes the interpretation of $p$-values and confidence intervals in the final model. 
Instead researchers should rely on theory to determine the model best suited for testing, and should they use sequential procedures for model selection, they should provide a replication using the final model only.

\subsection{Inference about Moderated Mediation}

Inferential methods are how we apply the findings from a sample of data to make inference about a population. Much of what I've discussed thus far relied on knowing if some parameter is zero or not. In truth, we do not ever know that a parameter is zero, but we can make inference about parameters based on estimates and estimates of sampling variability. There are two primary parameters that I'll focus on in this section: the index of moderated mediation and conditional indirect effects. Bootstrapping methods or Monte Carlo methods (as described in Chapters 1 and 2) can be used to generate confidence intervals for each coefficient and combinations of coefficients.

If $W$ is dichotomous, then the two values of $W$ can be plugged into the equation for the conditional indirect effect and bootstrapping methods can be used to generate a confidence interval for the difference between the two indirect effects. This is quite similar to the method proposed by Fairchild and MacKinnon (2009) for moderated mediation in between-subjects designs. Most of the time, this method is not needed as the difference between the two indirect effects will be equal to the index of moderated mediation. However, in the case of the most general model, this approach would be useful as the index of moderated mediation is not defined for such a model.

The parameters involved in conditional indirect effect and the index of moderated mediation can can be estimated using OLS regression or structural equation modeling. 
The estimates of the coefficients will be the same whether regression or SEM is used; however the estimates of the standard errors will differ by method, as structural equation modeling uses asymptotic standard error estimates and OLS does not (Hayes \& Scharkow, 2013; Hayes et al., 2017).

\subsubsection{Methods of Inference}

Inference for the conditional indirect effect and the index of moderated mediation can be conducted in a variety of ways. Early methodological work in moderated mediation for between-subjects designs recommended a piece-meal test, such that evidence of moderated mediation would be established if the total effect was significantly moderated, the paths that were allowed to be moderated were significantly moderated, and the paths that were not moderated were significant (Edwards \& Lambert, 2007; Muller et al., 2005; Preacher et al., 2007). However, Hayes (2015) suggested use of the index of moderated mediation, a quantification of the degree to which the indirect effect depends on the moderator. Ordinary least squares regression provides a point estimate for the index of moderated mediation. Confidence intervals for this index can be used for inference. This method creates a more parsimonious test of moderated mediation. Next I consider two methods for creating confidence intervals for the index of moderated mediation and conditional indirect effects: percentile bootstrapping and the Monte Carlo method.

Bootstrapping (as described in Chapter 2) can be used to conduct inference on the index of moderated mediation. For each bootstrap sample, an estimate of the index of moderated mediation is calculated, resulting in a distribution of estimates of the index of moderated mediation. This distribution can be used to calculate an 
estimate of the standard error of the index of moderated mediation, or percentiles of the distribution can be used to generate a confidence interval for the index of moderated mediation. Much research in the between-subjects domain suggests that percentile or bias-corrected bootstrapping methods perform very well, though biascorrected bootstrapping can have Type I error rates which are too high (Williams \& MacKinnon, 2008; MacKinnon et al., 2004; Hayes \& Scharkow, 2013; Biesanz et al., 2010). When the resampling is done in this within-subjects case, the resampling must be done at the individual level. The dependency among the observations must not be broken up, so all of the observations from a single individual must be sampled all together, meaning that each case that is resampled should include five measurements: the outcome in each instance, the mediator in each instance, and the moderator.

Another method for conducting inference is the Monte Carlo (MC) method. This method utilizes the normality of the individual paths in the conditional indirect effect or the index of moderated mediation (e.g., $\hat{a}_{1}$ and $\hat{b_{1}}$ from the first stage conditional process model). If we were generate an $\mathrm{MC}$ confidence interval for the index of moderated mediation for a first stage conditional process model, $a_{1} b_{1}$, there would be two distributions generated; one where values are generated from a normal distribution with mean $\hat{a_{1}}$ and standard deviation $\widehat{s_{a_{1}}}$ and the second with random numbers from a normal distribution with mean $\hat{b_{1}}$ and standard deviation $\widehat{s e_{b_{1}}}$. These values are multiplied by each other, resulting in an estimated sampling distribution of index of moderated mediation. From this distribution, the 2.5th and 97.5th percentile provide endpoints for a $95 \%$ confidence interval. Previous research in between-subjects mediation suggests that this method works well (Preacher \& Selig, 2012; Biesanz et al., 2010; Fritz \& MacKinnon, 2007; MacKinnon et al., 2004). 
As mentioned previously, there are many other methods for conducting inference for the indirect effect. I chose to describe percentile confidence intervals and Monte Carlo confidence intervals in this dissertation because they both respect the nonnormality of the indirect effect and can be used to provide a point estimate and confidence interval for the indirect effect. Chapter 4 includes three examples that all use the percentile bootstrap confidence interval for inference about the index of moderated mediation and conditional indirect effects.

\subsubsection{Probing Conditional Indirect Effects}

Bootstrapping or Monte Carlo methods can be used to estimate confidence intervals for conditional indirect effects. This can be done by choosing a specific value of $W$ and creating bootstrap confidence intervals for the linear function that defines the conditional indirect effect. Table 3.1 defines the conditional indirect effect for first-stage, second-stage, and first- and second-stage conditional process models.

By choosing values of the moderator and estimating and creating confidence intervals for the conditional indirect effect, we are essentially conducting the simple-slopes approach to probing conditional effects. The asymptotic standard errors in Table 3.1 could be used to generate a $z$-statistic and a $p$-value and a confidence interval. However, doing so assumes that the sampling distribution of the conditional indirect effect is normal. This is true in large samples, but not typically true in samples of the size usually collected in psychology (Stone \& Sobel, 1990). All asymptotic variances in Table 3.1 are based on second order Taylor expansions (i.e., the Delta Method). See the technical appendix in Preacher et al. (2007) for details. 
The Johnson-Neyman procedure can be used to probe conditional indirect effects using asymptotic standard error estimates. Similar to the simple-slopes approach this method relies on a normality assumption for the distribution of the indirect effect. An assumption known to be false in many realistic cases. Bootstrapping and Monte Carlo confidence intervals cannot be used to find the Johnson-Neyman transition points, because the Johnson-Neyman method relies on comparing to a critical value of a pre-specified sampling distribution. Bootstrapping and Monte Carlo confidence intervals estimate the sampling distribution of the indirect effect rather than assuming its shape. However, computing a variety of bootstrap or Monte Carlo confidence intervals along the range of the moderator could give a fairly clear sense about where the transition points are without assuming normality of the conditional indirect effect.

\subsection{Summary}

This chapter has described a variety of regression models which can be used to estimate conditional process models in two-instance repeated measures designs. I began with the most general model which allows the moderator $W$ to moderate the relationship between instance and the mediator, the mediator and the outcome, and the direct effect. Then I described how you can constrain this general model to get simpler conditional process models which are frequently used in the between-subjects literature: the first-stage conditional process model and second-stage conditional process model. For these simpler models, I showed how you could allow the direct effect to be moderated or constrain it to be constant across the moderator. For each model, I derived the conditional indirect effect and the index of moderated mediation where appropriate. I described how bootstrapping and Monte Carlo confidence intervals can 


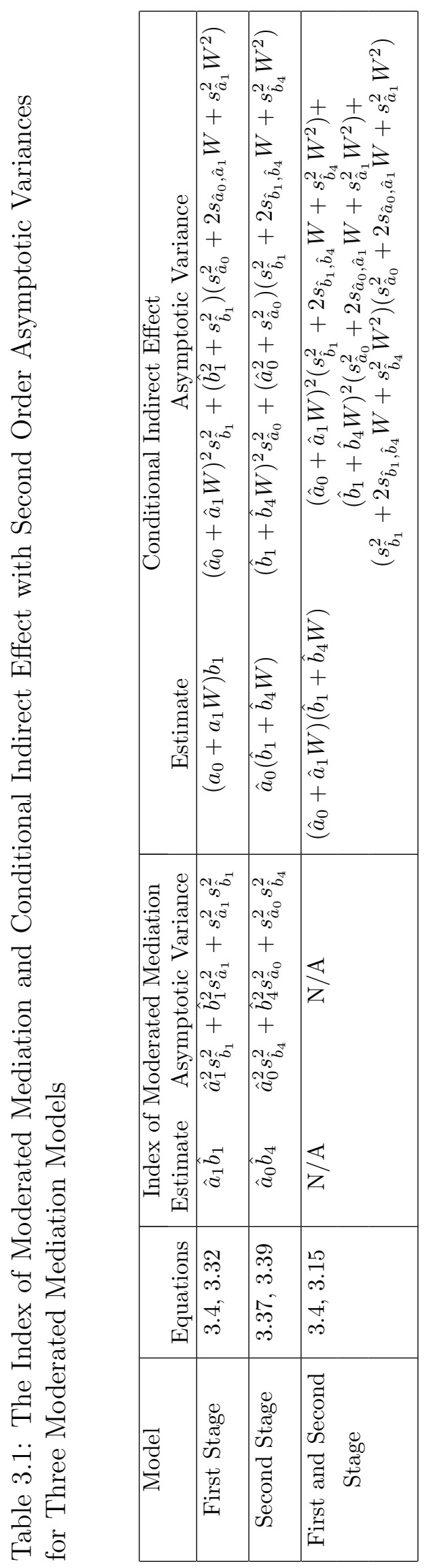


be used to make inference about the index of moderated mediation and conditional indirect effects. Additionally, Table 3.1 provides information about the asymptotic variances of the index of moderated mediation and conditional indirect effects. These variances can be used for hypothesis testing or the Johnson-Neyman procedure; however, the tests rely on an assumed normal distribution of the indirect effect which is not typically the case in small samples.

Much of what I have discussed in this chapter is the technical detail required to estimate conditional process models in two-instance repeated-measures designs. The next chapter describes a variety of applications of these models using data collected in psychology studies. This chapter will show better how to implement and interpret the models described in Chapter 3. 


\section{Chapter 4: Applications}

In this chapter, I provide examples of estimating the models described in Chap-

ters 3. The first example includes moderation on the first stage, the second example includes moderation on the second stage, and the final example includes moderation on all paths. These examples demonstrate the variety of different types of studies for which these models are appropriate. Example 1 comes from a study where all participants are measured before and after an intervention. All participants go through the same intervention. The moderator is a continuous variable which moderates the effect of time (assuming this is primarily due to the intervention) on the mediator. The second example is also a pre-post design, but half of the participants are in a control condition, and the other half are in a treatment condition. The treatment is included as a moderator of the path from the mediator to the outcome. This example exemplifies how data from a 2 (within) $\times 2$ (between) design can be analyzed using the conditional process models described in Chapter 3. The final example is a withinsubjects experimental design, where each participant is asked to rate two different stimuli. The order in which the stimuli are presented is used as a moderator of all paths to test whether order has an effect on the indirect and/or the direct effect.

These examples make the breadth of experimental designs which are suitable for using the conditional process models proposed in Chapter 3 clear. Additionally, these 
examples show the different types of research questions and hypotheses that can be answered with these types of analyses.

\subsection{Example 1: Lasselin et al. (2016)}

Lasselin et al. (2016) investigated whether baseline inflammation moderates the effectiveness of behavioral treatment for chronic pain. They were particularly interested in whether the treatment was less effective for individuals with higher baseline inflammation. Patients $(N=40)$ with chronic pain were recruited to the study, which involved 12 weekly sessions of behavioral treatments for chronic pain. Participants reported their pain before starting treatment and after completion of the sessions. Baseline inflammation was measured by taking assays of two pro-inflammatory markers (IL-6 and TNF- $\alpha$ ). The concentrations of these markers were log transformed to improve linearity and combined using principal components analysis. Lasselin et al. (2016) found evidence that the effect of treatment on pain was moderated by inflammation. In the paper, they did not investigate any potential mechanisms. However, they did measure some potential mechanisms. Lasselin et al. (2016) suggested that psychological inflexibility as measured by the Psychological Inflexibility in Pain Scale (PIPS; Wicksell, Renofalt, Olsson, Bond, \& Melin, 2008) might act as a mechanism by which the treatment could impact pain. Lasselin et al. (2016) suggest that behavioral therapy might help individuals be more psychologically flexible, which could decrease their pain. In this example, I investigate if psychological inflexibility might mediate the effect of behavioral treatment on pain. After consulting the primary author about what theoretical model would make the most sense, I allowed inflammation to moderate the effect of treatment on psychological inflexibility as well as the direct 
effect from treatment to pain. The analyses in this dissertation are different from the analyses in Lasselin et al. (2016). A single outlier was dropped for all analyses in this dissertation. All tests are conducted at $\alpha=.05$. For the original analysis and other variables measured in the study, see Lasselin et al. (2016).

This example is a first-stage conditional process model as described in Section 3.2, with the direct effect also moderated. In this model the the focal predictor is treatment (though it is confounded with the passage of time), the mediator is psychological inflexibility, and the outcome is pain. The psychological inflexibility measure (PIPS) is the sum of 12 Likert type items ranging from 1 to 7 , where higher scores indicate higher inflexibility. In the pre-measurement of the PIPS, the average was $56.49(S D=10.72)$. In the post-measurement of the PIPS the average was 50.78 $(S D=16.93)$. Pain is measured with one Likert type item scored from 1 to 6 . In the pre-measurement of the pain scale the average was $4.33(S D=0.92)$. In the postmeasurement the average was $4.13(S D=1.28)$. Inflammation scores were calculated using a principal component analysis, so the average is 0 and standard deviation is 1 .

For each step of the model, I fit the models to the data as outlined in Chapter 3. Mplus code for estimating the parameters from this model can be found in Appendix A. First I estimated the difference in the mediators (Equation 3.4). This is a model of the difference in psychological inflexibility from Time 1 to Time 2 , as predicted by inflammation. The resulting estimate is

$$
\widehat{M_{2 i}-M_{1 i}}=-5.70+4.16 W_{i}
$$

The intercept of this equation can be interpreted as the expected change in pain (Time 2 - Time 1) for an individual who scored 0 on inflammation (i.e., they are 
exactly at the sample mean). Someone who is average on inflammation is expected to decrease in psychological inflexibility over the treatment period by 5.704 units $(t(38)=-3.29, p=.002)$. The coefficient for $W$ indicates that as inflammation increases by 1 unit, the difference in psychological inflexibility is expected to increase by 4.16 units. This means that as inflammation gets higher, the difference between psychological inflexibility from Time 1 to Time 2 will actually get smaller. So people who are higher on inflammation will see a smaller reduction in psychological inflexibility. In fact, individuals who score a 1.37 on inflammation are expected to see no difference in psychological inflexibility from Time 1 to Time 2. Of the observed individuals, $10 \%$ scored above 1.37. Using the Johnson-Neyman procedure, I examined for what range of inflammation are there significant differences in psychological inflexibility. There was one solution, 0.44 which suggests that for individuals with lower than 0.44 standard deviations below the mean of inflammation there was significant improvement in psychological flexibility. For all individuals with scores higher than 0.44 there was no significant effect of treatment on psychological flexibility. Additionally, this suggests that even in the very high ranges of observed inflammation there was no point at which treatment began to have a significant negative effect on psychological flexibility (i.e., inflexibility did not get significantly higher over the course of treatment). These results together indicate there there is significant moderation of the effect of treatment on psychological inflexibility, where individuals who are lower on inflammation see greater decreases in psychological inflexibility. Whereas those who are higher on inflammation see smaller or no decreases in psychological inflexibility. 


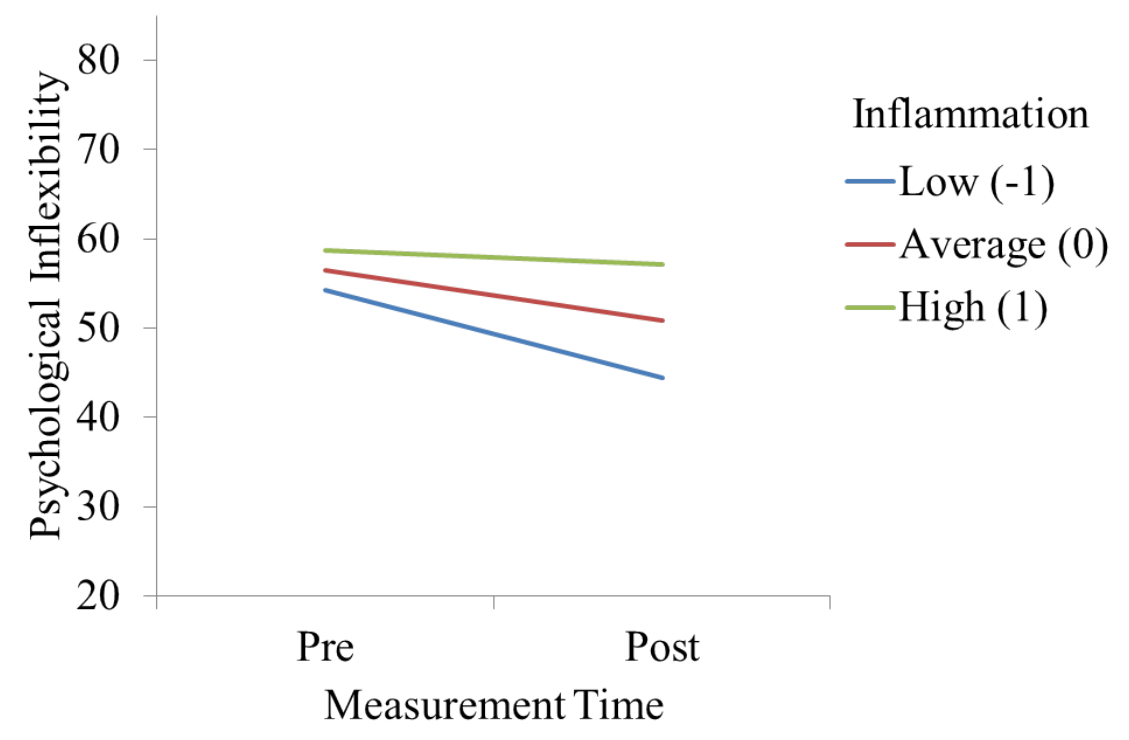

Figure 4.1: Plot of interaction between time and inflammation on psychological inflexibility.

For assessing moderated mediation, the important estimate from this equation is $\theta_{X \rightarrow M}(W)$ which is the conditional effect of time (or treatment) on the mediator. This effect is a linear function of the moderator. In this model the estimate of $\theta_{X \rightarrow M}(W)$ is $-5.704+4.160 \mathrm{~W}$. This estimate will be used to calculate the conditional indirect effect. Additionally, the estimate of $a_{1}(4.160)$ will be used to calculate the index of moderated mediation.

The next model is for the difference in the outcomes (pain), which is a linear function of the difference in the mediators (psychological inflexibility), the grand mean centered average of the mediators, and (because we're allowing the direct effect to be moderated) the moderator is also included in the model (See Equation 3.32). The estimated model in the Lasselin data is 


$$
{\widehat{Y_{2 i}-Y_{1 i}}}=-.09+0.02\left(M_{2 i}-M_{1 i}\right)-0.01{\frac{M_{2 i}+M_{1 i}^{*}}{2}}^{*}+0.35 W_{i}
$$

I use the $*$ superscript to indicate that the average of the repeated-measurements of the mediators has been grand-mean centered. Based on this equation we can estimate the conditional direct effect $\theta_{X \rightarrow Y}\left(M_{A}=\overline{M_{A}}, W\right)$ as $-0.09+0.35 W$. That is, for an individual who is at the sample mean on the average of the mediators, and who has a score of zero on inflammation, the direct effect of treatment on pain is expected to be $\hat{b}_{0}=-0.09$. This effect is not significantly different from zero $(t(36)=-0.39, p=.70)$. Additionally, as inflammation increases by one unit, the direct effect is expected to increase by $\hat{b}_{3}=0.35$ units, but this effect is also not significantly different from zero $(t(36)=1.68, p=.10)$. Thus, there is not significant evidence that the direct effect of treatment on pain is moderated by inflammation.

Another important part of this model is the degree to which the mediator (psychological inflexibility) predicts the outcome (pain). Based on the estimated model, a one unit increase in the difference in psychological inflexibility results in a $b_{1}=0.02$ unit increase in the difference pain, but this effect is not significantly different from zero $(t(36)=0.93, p=.36)$. There was also not significant evidence that the relationship between the mediator and the outcome differed across time points $\left(b_{2}=\right.$ $-0.01, t(36)=-0.39, p=.71)$. This suggests that although theoretically, psychological inflexibility should be a strong predictor of pain, in these data there is not much evidence that psychological inflexibility predicts pain. This could mean that psychological inflexibility is unlikely to serve as a mediator of the relationship between treatment and pain. However, it is important to note that the indirect effect is made up of two effects: the effect of $X$ on $M$ and the effect of $M$ on $Y$. Just because an 
inferential test for the effect of $M$ on $Y$ does not suggest there is an effect does not mean that we should reject the hypothesis of mediation. Relying on multiple fallible inferential tests to determine mediation or moderated mediation is less parsimonious and more prone to error than relying on a single test on the indirect effect or the index of moderated mediation.

To estimate the indirect effect, take the product of the conditional effect of instance on $M$ and the effect of $M$ on $Y,\left(\hat{a}_{0}+\hat{a}_{1} W\right) \hat{b}_{1}$. Plugging in estimates of each of these paths gives $-0.11+0.08 \mathrm{~W}$. We can use percentile bootstrapping to estimate confidence intervals for the conditional indirect effect at a variety of values of the moderator. See Table 4.1 for estimates fo the conditional indirect and direct effect at the mean, plus, and minus one standard deviation of the moderator. The complete model results are represented in Figure 4.2.

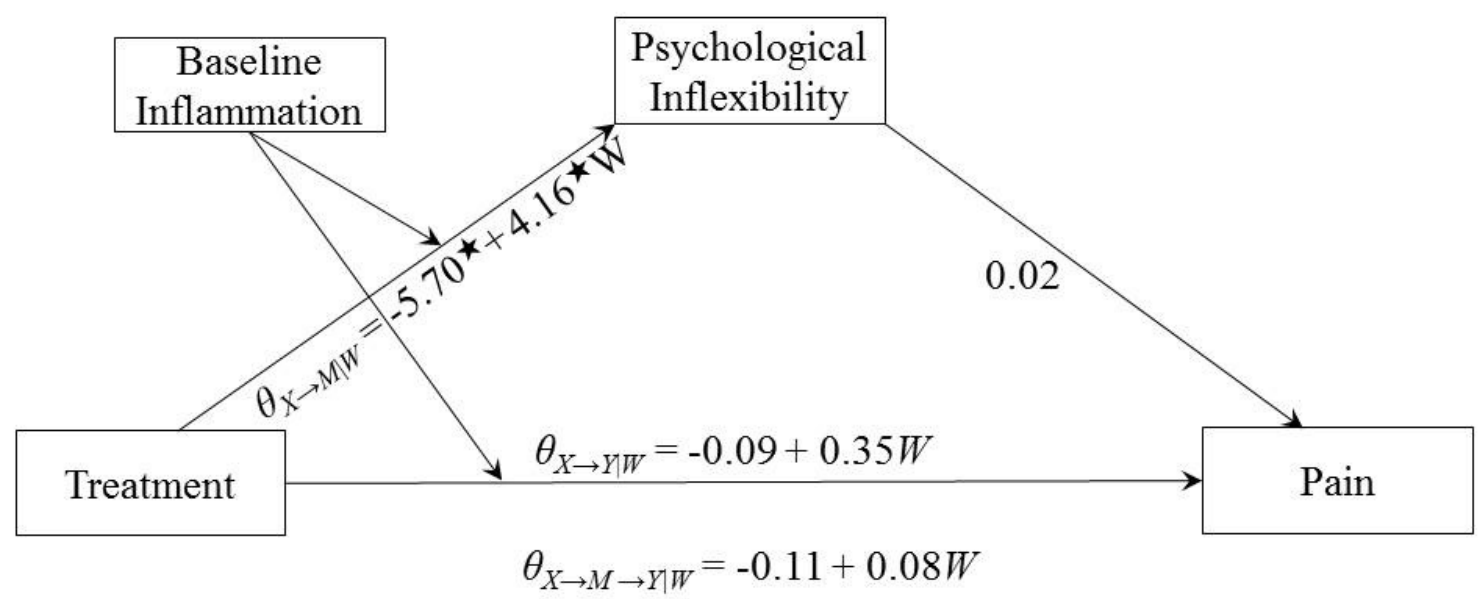

Figure 4.2: Diagram for Lasselin et al. (2016) First-Stage Conditional Process Model. $\star$ indicates statistically significant coefficients at $\alpha=.05$. 
Table 4.1: Conditional Effects of Treatment on Pain with 95\% confidence intervals

\begin{tabular}{|c|c|c|c|c|c|c|}
\hline Moderator Value & \multicolumn{3}{|c|}{ Indirect Effect } & \multicolumn{3}{|c|}{ Direct Effect } \\
\hline-1.01 & -0.19 & {$[-0.56$} & $0.17]$ & -0.44 & {$[-1.10$} & $0.22]$ \\
\hline 0.00 & -0.11 & {$[-0.34$} & $0.09]$ & -0.09 & {$[-0.55$} & $0.37]$ \\
\hline 1.01 & -0.03 & {$[-0.17$} & $0.07]$ & 0.29 & {$[-0.32$} & $0.85]$ \\
\hline
\end{tabular}

For none of the probed values of the mediator did the confidence interval for the indirect effect exclude zero. This means that for individuals ranging from one standard deviation above the mean on inflammation to one standard deviation below, there was not sufficient evidence that psychological inflexibility serves as a mediator of the relationship between treatment and pain. Based on this table it is clear the hypothesized direction of change is supported by the data: as inflammation increases the indirect effect is also increasing (becoming less negative). However, to formally test this hypothesis, we need a bootstrap confidence interval for the index of moderated mediation, $a_{1} b_{1}$.

The index of moderated mediation in this data set is estimated as 0.08 with a bootstrap confidence interval of $(-0.07,0.25)$. This means that the indirect effect of treatment on pain through psychological inflexibility is expected to increase by 0.08 units with every one unit increase in inflammation. However, the confidence interval includes zero, which suggests that a change of zero is still a plausible value. Thus we cannot rule out that the index of moderated mediation is zero. This means there is insufficient evidence to claim moderated mediation based on these data. 
There is not sufficient evidence from this study to suggest that psychological inflexibility serves as a mediator of the influence of treatment on pain across a variety of values of baseline inflammation. Additionally, we did not find sufficient evidence that the indirect effect depends on inflammation. Nor did we find that the direct effect of treatment on pain was moderated by inflammation. The direction of all the effects, however, are in line with the predictions of Lasselin et al. (2016). It is worth noting that the sample size for this study is quite small (40 individuals), and this size of a sample is unlikely to be sufficient to detect a small indirect effect or small moderation effects.

\subsection{Example 2: Bell, Shader, Webster-Stratton, Reid, Beauchaine (2017)}

Bell, Shader, Webster-Stratton, Reid, and Beauchaine (2017) investigated how a family behavioral intervention, call the "Incredible Years," might impact externalizing symptoms of children with ADHD through parenting behaviors. In this intervention, parents learn appropriate responses to their children's behaviors, and children do tasks that help them learn more emotion regulation skills. The training is administered in 20 two-hour weekly sessions. In this study, families were randomly assigned to either receive the treatment starting at Week 1 or a delayed treatment, starting at Week 10. Each family was measured before the intervention period (Week 0), and then again 20 weeks later. So at 20 weeks the treatment group had received 40 hours of treatment, and the delayed group had received 20 hours of treatment. The mediators of interest in the study were positive parenting and negative parenting. I will focus on positive parenting. 
The outcome variable I will focus on is cardiac pre-ejection period (PEP) reactivity. PEP is a physiological measure that is assumed to be related to central nervous system reward dysfunction. This type of dysfunction can put children at risk for a variety of externalizing disorders including ADHD. Higher in PEP reactivity is considered to be more "typical." So the intervention is meant to increase PEP reactivity over time. PEP reactivity was measured before the intervention period and at the end of 20 weeks.

The mediator, positive parenting, was assessed during a 30 minute free-play period. The play period was videotaped, and trained research assistants used the Dyadic Parent-Child Interaction Coding System (Robinson \& Eyberg, 1981) to code for positive and negative parenting. Positive parenting was measured before the intervention and at the end of 20 weeks.

The treatment variable in the data is coded so those who received the treatment immediately are coded as 1 , and those who were on the delayed are coded as zero. Again remember that at the "post" measurement the treatment group received two times the amount of treatment that the delayed group received. The delayed group did, however, receive some of the treatment between pre- and post-measurements, meaning we would expect them to change somewhat from before the intervention to after. Table 4.2 include descriptive statistics for the measured variables split by experimental condition.

I conducted a second-stage conditional process analysis. In this analysis time is considered the independent variable, positive parenting is the mediator, and PEP reactivity is the outcome. Experimental condition (immediate vs. delayed) moderates the relationship between positive parenting and PEP reactivity. The direct effect is 
Table 4.2: Measured Variables by Time and Condition: Mean (Standard Deviation)

\begin{tabular}{|cc|cc|}
\hline Outcome & Condition & Pre & Post \\
\hline PEP Reactivity & Delayed & $0.74(3.98)$ & $0.75(3.59)$ \\
& Immediate & $1.37(3.53)$ & $1.35(3.84)$ \\
\hline Positive Parenting & Delayed & $22.03(13.12)$ & $27.75(16.94)$ \\
& Immediate & $23.39(18.45)$ & $39.75(24.74)$ \\
\hline
\end{tabular}

moderated, as there may be other reasons for condition to impact PEP reactivity other than through parenting. I used Equations 3.37 and 3.39 to estimate the model. Mplus code for estimating this model can be found in Appendix B. I began by estimating the equation for the difference in the mediators (positive parenting score). All differences were calculated by subtracting the pre-measurement from the postmeasurement. Positive difference scores indicate that the measure is higher at Time 2 than Time 1. Because this path is not moderated Equation 3.37 is used. This equation only estimates an intercept, which based on the data is 10.99. This means that, on average, parents scored 10.99 points higher on the positive parenting scale after the intervention than before. This estimate includes individuals in both the experimental condition and the delayed control condition. For reference, positive parenting scores ranged from 2 to 112 at pre-treatment with an average of 22.70 and standard deviation of 15.91. At post-treatment scores ranged from 1 to 110 with an average of 33.69 and standard deviation of 21.90 .

Next, I used Equation 3.39 to estimate the relationship between parenting and PEP reactivity, and whether this relationship is moderated by experimental condition. The estimated model in the data is 


$$
\begin{aligned}
\widehat{Y_{2 i}-Y_{1 i}=} & -0.18+0.01\left(M_{2 i}-M_{1 i}\right)-0.03 \frac{M_{2 i}+M_{1 i}}{2}+1.25 W_{i} \\
& -0.08 W_{i}\left(M_{2 i}-M_{1 i}\right)+0.06 W_{i} \frac{M_{2 i}+M_{1 i}}{2}
\end{aligned}
$$

In this equation, the intercept is the predicted difference in PEP reactivity for a child who's parents showed no difference on parenting pre vs. post, are average on positive parenting, and are in the delayed onset condition. The intercept estimate is -0.18 , and this effect is not significantly different from zero $(t(93)=-0.23, p=0.82)$. The slope for the difference in the mediators suggests that for individuals who are in the delayed onset condition, families who differ by one unit on change in parenting are expected to increase by 0.01 units more on PEP reactivity $(t(98)=0.29, p=.77)$. This means that for those families in the delayed condition, positive parenting is not significantly related to PEP reactivity. The slope for the average of the mediators suggests that for individuals who are in the delayed condition, families who differ by one unit on average positive parenting the PEP reactivity change is expected to decrease by 0.03 units $(t(98)=-0.46, p=.64)$. This means that among those families in the delayed condition, there is no significant evidence that relationship between positive parenting and PEP reactivity differs from pre- to post-treatment.

The coefficient for $W$ means we expect individuals in the treatment condition to show a 1.25 larger difference in PEP reactivity compared to those in the delayed onset condition, for families who show no difference on positive parenting and are at the sample mean for average positive parenting. This an estimate of the degree to which condition moderates the direct effect. It is not significantly different from zero $(t(93)=1.09, p=.28)$. 
The coefficient for the product of the mediator differences and the moderator, -0.08 , indicates the degree to which the relationship between the mediator and the outcome is moderated by $W$. The relationship between the mediator and the outcome is expected to be 0.08 units weaker for those in the intervention condition, but this effect is not significantly different from zero $(t(93)=-1.62, p=0.11)$. This coefficient is important for estimating the indirect effect as well as the index of moderated mediation. Again, inferential tests of the individual paths do not determine mediation or moderated mediation. It is better to use a single test on the parameter of interest to make inferential decisions about these types of hypotheses. Just because the $b_{1}$ path is not statistically significant does not mean there is not evidence of mediation or moderated mediation.

The coefficient for the product of the mediator averages and the moderator indicates whether the degree to which the relationship between $M$ and $Y$ varies from pre- to post- measurements differs across conditions. Based on this estimate the difference between the $M-Y$ relationship (pre vs. post) is 0.05 units larger for those in the treatment condition than those in the delayed condition. This effect is not significantly different from zero $(t(93)=0.75, p=.46)$. The complete estimated model is represented in Figure 4.3.

To estimate the indirect effect, I took the product of the effect of instance on $M$ and the conditional effect of $M$ on $Y, \hat{a}_{0}\left(\hat{b}_{1}+\hat{b}_{4} W\right)$. Plugging in estimates of each of these paths gives $0.14-0.90 \mathrm{~W}$. I used percentile bootstrapping to estimate confidence intervals for the conditional indirect effect at the two coded values of the 


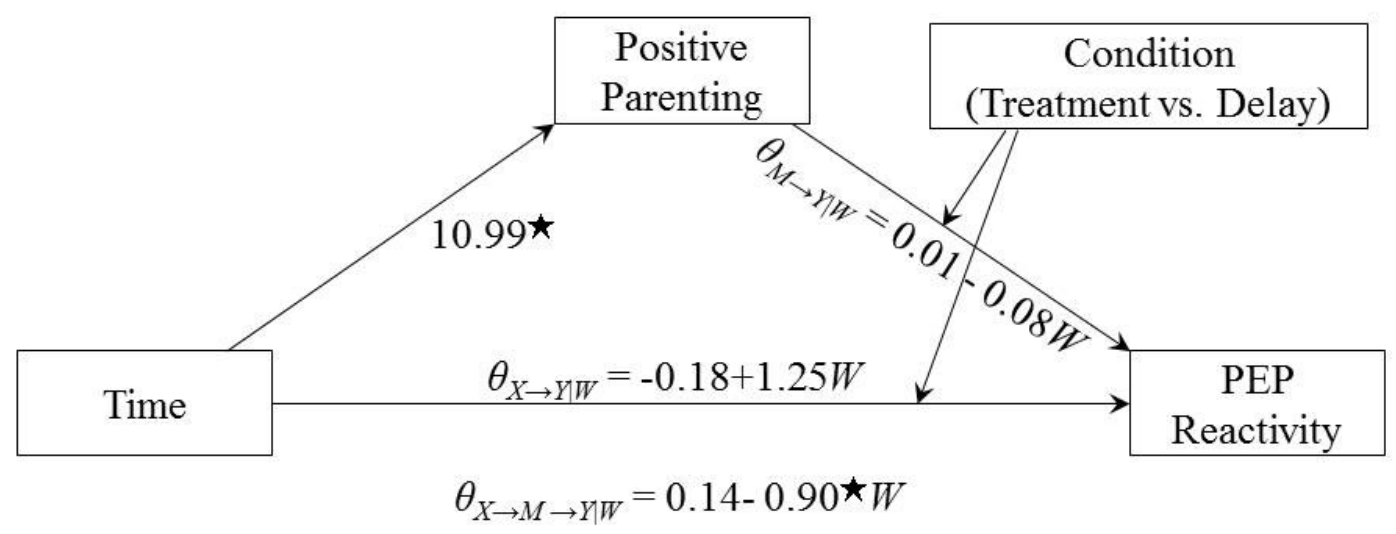

Figure 4.3: Diagram for Bell et al. (2018) Second-Stage Conditional Process Model. $\star$ indicates statistically significant coefficients at $\alpha=.05$.

Table 4.3: Conditional Effects of Time on PEP Reactivity with 95\% confidence intervals

\begin{tabular}{|c|c|c|c|c|c|c|}
\hline Moderator Value & \multicolumn{3}{|c|}{ Indirect Effect } & \multicolumn{3}{|c|}{ Direct Effect } \\
\hline 0 (Delayed Intervention) & 0.1383 & {$[-0.4208$} & $0.7960]$ & -0.18 & {$[-1.7469$} & $1.3852]$ \\
\hline 1 (Immediate Treatment) & -0.7661 & {$[-1.8796$} & $-0.1341]$ & 1.0656 & {$[-0.5734$} & $2.7047]$ \\
\hline
\end{tabular}

moderator. See Table 4.3 for estimates fo the conditional indirect and direct effect in each of the two conditions.

For individuals in the control condition, there was not a significant indirect effect of time on PEP reactivity through positive parenting. However, for those in the treatment condition there was a decrease in PEP reactivity of 0.77 over time through positive parenting, and the confidence interval for this estimate did not include zero. Both conditional direct effects were not significantly different from zero, 
and remember there was no strong evidence that the direct effect was moderated by condition.

The hypothesized direction of change was not supported by the data. In fact the opposite direction was supported: where those in the treatment condition see negative indirect effects on PEP reactivity through positive parenting. However, just because one indirect effect is significantly different from zero and the other is not does not mean they are necessarily different from each other. It is useful to test if the indirect effect in the treatment condition is different from the indirect effect in the control condition. This hypothesis can be tested using the index of moderated mediation and a bootstrap confidence interval for the index. The index of moderated mediation is $a_{0} b_{4}$.

The estimate of the index of moderated mediation in this data set is -0.90 with a bootstrap confidence interval of $[-2.27,-0.07]$. This means that the indirect effect of time on PEP reactivity through parenting is 0.90 units lower in the treatment condition. The confidence interval does not include zero, so we take this to be sufficient evidence that these two indirect effects are significantly different from each other.

Based on this analysis it seems there is worsening PEP reactivity over time through positive parenting for individuals in the treatment condition. This decline through positive parenting is significantly larger than for those in the delay condition. Overall, this suggests that there are some potential negative outcomes occurring through this intervention for the children. However, when considering an interventions effectiveness it is important to consider the overall effects. The total effect on PEP reactivity is not significant in either condition, nor is it significantly moderated. Looking at the conditional direct and indirect effects this is not surprising, as they are of opposite 
signs in each condition, suggesting there may be some beneficial mechanisms through which time effects PEP reactivity, and some detrimental mechanisms through which this effect occurs. Finding interventions which bolster the beneficial relationships may help researchers have a greater impact on PEP reactivity through other interventions in the future.

\subsection{Example 3: Montoya, Master, Cheryan (2013)}

One particular situation where using conditional process analysis may be helpful is investigating order effects in a two-instance repeated measures design where mediation is of primary interest. When conducting a mediation analysis with a two-instance repeated-measures designs, the researcher may worry that the observed effects are influenced by the order of stimuli presentation. It is quite common in within-subjects experimental designs to randomize order of presentation of the stimuli, and if this is done, order can be used as a between-person moderator and allowed to moderate all paths in the mediation model, using the general model outlined in Chapter 3. It is extremely important to note, that this type of analysis cannot be used to support the claim that there are no order effects. This would involve accepting the null hypothesis based on a "non-significant" finding. Other methods such as equivalence testing procedures would need to be used to make claims that order effects are near zero. Equivalence testing procedures could use the models presented, however the procedures used to make inference would be difference. If there are order effects the following procedures will help researchers identify that they exist.

The data for this example come from Montoya, Master, and Cheryan (2013). In this study, the researchers investigated if group work in computer science classes 
might increase women's interest in computer science by increasing their perception that computer science is a communal field. This should then increase their interest in computer science. In the study, 51 female college undergraduates read two syllabi for computer science courses. One syllabus was for an introductory computer science course that included group projects throughout the course. The other syllabus was for an introductory computer science course which included individual projects throughout the course. The syllabi also differed in the name of the instructor (both male sounding names) and the computer language (Java vs. Python). After reading both syllabi, the participants then responded to questions about the first syllabus they saw (Class A), next answered questions about the second syllabus they saw (Class B). The order of the group work and individual work syllabi was randomized across participants. Participants rated how interested they were in taking each class using three 7-point Likert type questions (per class). An example of a question for interest is "How interested are you in taking Class A?" Additionally, participants responded to five 7-point Likert type questions about how communal they thought the class would be. An example item for the communal questions is "Taking this class would assist me in helping others." Each of the communal questions were of the same type but with the goal replaced. The other goals were serving the community, working with others, connecting to others, and caring for others.

The goal of this study was to examine if women would be more interested in a computer science class with group work, and if there is an indirect effect of group work on interest through communal goals. This question as it stands is a question of mediation, and no moderation is needed. However, because the design is withinsubjects there is a chance that there could be order effects and these effects could 
impact the results of the study. I will first review the results of the mediation analysis then examine a model which allows order to moderate all paths in the mediation model.

The mediation model was estimated in MEMORE (Montoya \& Hayes, 2017) using Equations 2.3, 2.6, and 2.9. All differences were constructed such that scores from the individual class were subtracted from the group class. Thus, a positive difference means that scores are higher in the group work class, and a negative difference means that the scores are higher in the individual work class. The estimate of the total effect, or the overall effect of group work on interest, was $0.37(t(50)=1.33, p=$ 0.19). Though the effect is in the predicted direction, where female students are more interested in the group work class than the individual work class, the effect was not significantly different from zero at $\alpha=0.05$. The effect of group work on communality was quite strong $(a=2.29, t(50)=9.21, p<.001)$. Additionally, a one unit increase in communality predicted a 0.59 unit increase in interest $(t(48)=$ $4.38, p<.001)$. There was no significant evidence that the effect of communality on interest differed across conditions $(d=-0.55, t(48)=-1.27, p=0.21)$. Finally, the direct effect was significantly negative, suggesting that controlling for communality there was a negative impact of group work on interest $\left(c^{\prime}=-.98, t(48)=-2.53, p=\right.$ 0.01 ). The overall indirect effect was 1.35 , meaning that through the mechanism of communality, women were 1.35 units more interested in the group work class than the individual work class. A bootstrap confidence interval for this effect did not include zero $(0.69,1.95)$. The results of this study support the conclusion that group work might increase interest through increasing the perception that the courses (and perhaps the field) are more communal; however, the negative direct effect suggests 
there may be other mechanisms at play which have an overall negative effect of group work on interest controlling for communality. The results of the estimated mediation model are represented in Figure 4.4 .

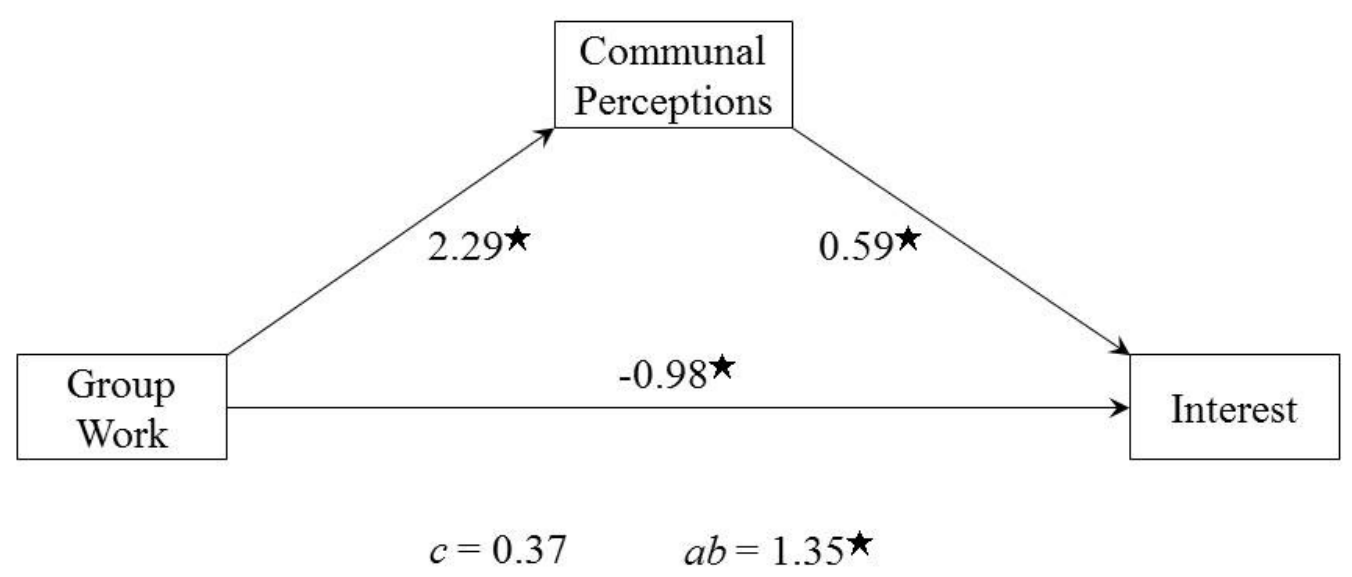

Figure 4.4: Diagram for Montoya et al. (2013) Mediation Model. $\star$ indicates statistically significant coefficients at $\alpha=.05$.

As mentioned, the order of the presentation of the classes could potentially impact the results of the study. Though all participants read both of the syllabi at the beginning of the study, before answering any questions, they answered questions about the syllabi in groups and thus perhaps the order in which the participants thought about the different classes could potentially impact the proposed mechanisms of the effect. To test for this I've allowed syllabus order to be a moderator of all the paths in the mediation analysis. This model corresponds to estimating Equations 3.4 and 
3.15. This means that the $a$-path, $b$-path, and the direct effect are all moderated. Based on this model we can estimate the conditional direct and indirect effects as well as test whether the indirect and/or direct effects are significantly different across the two order presentations. Mplus code for estimating this model can be found in Appendix C. Order is coded such that those who saw the group work class first are coded as 0 , and those who saw the individual work syllabus first are coded as 1 .

The estimated equations are:

$$
\begin{aligned}
\widehat{M}_{2 i}-M_{1 i}= & 1.63+1.20 W_{i} \\
\widehat{Y_{2 i}-Y_{1 i}=} & -1.16+0.54\left(M_{2 i}-M_{1 i}\right)-0.10 \frac{M_{2 i}+M_{1 i}}{2}+0.83 W_{i} \\
& -0.08 W_{i}\left(M_{2 i}-M_{1 i}\right)-1.28 W_{i} \frac{M_{2 i}+M_{1 i}}{2}
\end{aligned}
$$

I use the $*$ superscript in the equations to indicate that the average of the repeatedmeasurements of the mediators has been grand-mean centered. Based on this analysis, the conditional estimate of the effect of group work on communality for those who saw group work first was $1.63, t(49)=4.64, p<.001$. Additionally, the effect of group work on communality was moderated, such that those who saw individual work first saw a larger effect of group work on communality $\left(a_{1}=1.20, t(49)=2.52, p=0.01\right)$. This may be because those who saw individual work first answered questions about the group work class after being reminded how they might feel in a "typical" computer science class where there is not group work. The contrast between the two may become more extreme after first answering questions about the individual work class, then considering the class with group work. 
In the model for the difference in $Y$ 's, the relationship between communality and interest is positive for those individuals in the group work first condition $\left(b_{1}=\right.$ $0.54, t(45)=2.77, p=0.01$. Additionally there is not significant evidence that the relationship between communality and interest is moderated by presentation order $\left(b_{4}=-0.08, t(45)=-0.28, p=.77\right)$. There is no evidence that the effect of communality on interest varies between group work and individual work classes $\left(b_{2}=-0.10, t(45)=-0.17, p=.87\right.$; i.e., group work does not moderate the effect of communality on interest). Additionally presentation order does not significantly moderate the degree to which group work moderates the relationship between communality on interest $\left(b_{5}=-1.28, t(45)=-1.43, p=.16\right)$. The estimated direct effect for individuals in the group work first condition is $-1.16, t(45)=-2.47, p=.02$. Additionally there is no significant evidence that the direct effect is moderated by presentation order $\left(b_{3}=0.83, t(45)=0.99, p=.33\right)$. It's worth noting that the conditional direct effects were both negative, but only significant in the group work first condition (Group work first: $-1.16, p=0.02$; Individual work first: $-.33, p=.64$ ). The estimated moderated mediation model is represented in Figure 4.5.

The estimated indirect effect is the product of the conditional effect of group work on communality and the conditional effect of communality on interest. In the group work first condition the estimated indirect effect is 0.88 (95\% Bootstrap CI $=$ -.1140, 1.59). When students read about the individual work class first, the estimated indirect effect is 1.29 (95\% Bootstrap CI $=0.33,2.48)$. The difference between these two effects is 0.41 (which is the index of moderated mediation). A $95 \%$ bootstrap confidence interval for this parameter includes zero $(-1.97,0.76)$, suggesting that there is not significant evidence that the indirect effect depends on presentation order. 
Remember that these methods cannot be used to support the claim that there are no order effects, but rather equivalence testing methods would need to be used to support such a claim.

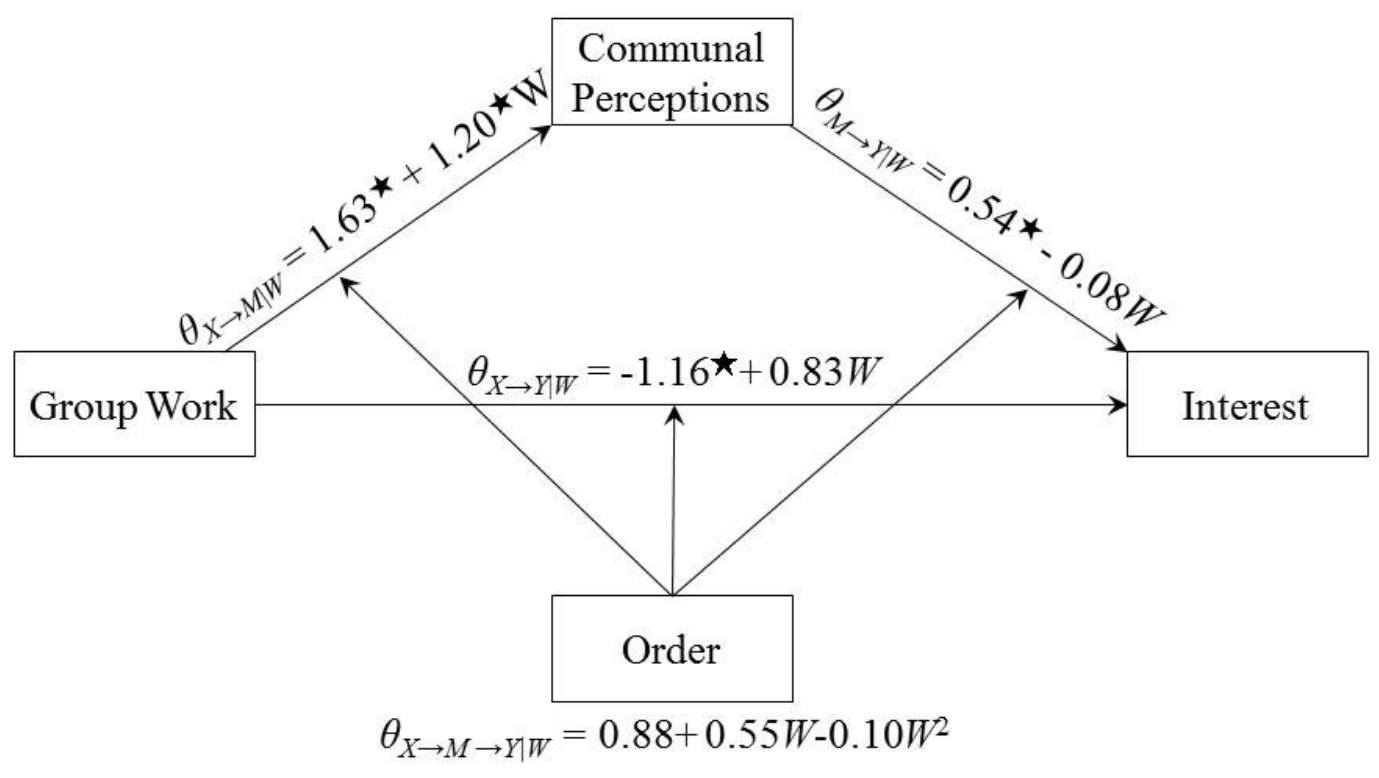

Figure 4.5: Diagram for Montoya et al. (2013) Conditional Process Model. $\star$ indicates statistically significant coefficients at $\alpha=.05$.

Overall, this analysis suggests that there are order effects for the path from group work to communality. The effect of group work was stronger for participants who rated the individual work class first. However, neither the indirect effect nor the direct effect showed significant evidence of moderation. All conditional effects were 
in the same direction, just stronger for one order than the other. This is comforting in that there are not reversed effects for one order compared to another.

One note on this analysis: When the moderator is dichotomous, another option for centering the average of the mediators would be group mean centering, based on the moderator groups (e.g., order). The centering choice affects the estimate of the direct effect, which is conditional on the centered value of the average of the mediators. When grand-mean centering is used, the direct effect is conditional on the overall average, but when group mean centering is used the direct effect is conditional on the group average. When group-mean centering is used, the conditional direct effect exactly replicates the results of a mediation analysis with just the individuals in that group. When the average is grand-mean centered, all other point estimates exactly replicate the results of the mediation analysis, but the conditional direct effect does not. Hayes, Montoya, and Rockwood (in prep) discuss the group-mean centering approach more in-depth. I use grand-mean centering as this is appropriate for both dichotomous and continuous moderators. 


\section{Chapter 5: Alternative Analytical Approaches}

In this chapter, I discuss alternative methods for evaluating conditional process models in repeated measures designs. I will describe how the first-stage conditional process model described in Chapter 3 is related to methods for testing mediation using a 2(within) X 2(between) design as described by MacKinnon (2008) and Valente and MacKinnon (2017). I will describe the assumptions needed for the models from Valente and MacKinnon (2017) to be equivalent to the first stage conditional process model described in Chapter 3. Two particularly popular alternative methods include multilevel models and structural equation models. Some work has been done in the area of multilevel modeling for assessing moderated mediation. I will connect the framework proposed for two-instance repeated measures designs to those described for 1-1-1 moderated mediation models. Little has been discussed in terms of assessing moderated mediation in structural equation models for repeated measures designs, however I will discuss latent-difference score models, latent-growth curve models, and cross-lag panel models. These models have all been used for assessing mediation in repeated-measures designs. I discuss some of the strength of these models and when they might be most appropriate for use in assessing moderated mediation in repeated measures designs. 


\subsection{Approaches to 2 (Within) X 2 (Between) Designs}

Valente and MacKinnon (2017) discussed four models for assessing mediation in pre- post-test control group designs. In these designs, individuals are measured on the proposed mediator and proposed outcome before random assignment to the treat-

ment. After a period of time (typically after treatment is complete) the mediator and outcome are measured again. The conditional process models proposed in Chapter 3 would be appropriate for these types of designs. The example in Section 4.2 was a pre-post design and analyzed using a second-stage conditional process model. In this section I discuss the models proposed by Valente and MacKinnon (2017), how these models connect to first-stage conditional process model in Chapter 3 defined by Equations 3.4 and 3.32, and compare these different approaches while discussing what some of the advantages and disadvantages of each approach would be.

\subsubsection{Models for Pre- Post-Test Control Group Designs}

Valente and MacKinnon (2017) compare four models for assessing the indirect effect of a randomized treatment on an outcome through a mediator for studies which use a pre- post-test control group design. Note that these models focus on the indirect effect of the randomized treatment, rather than, as we have been considering throughout this dissertation, the indirect effect of the repeated-measures factor. In this type of design the repeated-measures factor would be time. The four models they consider are the ANCOVA Model, a Difference Score Model, a Residualized Change Model, and a Cross Sectional Model. I will describe each of these individually, then connect these models back to the first-stage conditional process model proposed in Chapter 3 . I will present each model with equations that use the difference score as the outcome 
variable, in order to more easily connect the models to the proposed models from Chapter 3. In each equation I will use the subscript A to refer to estimates from the ANCOVA Model, D to refer to estimates from the Difference Score Model, $\mathrm{R}$ to refer

to the Residualized Change Model, and C for the Cross Sectional Model. Much of the discussion of these models relies on the concept of stability. Kenny (1979) defines stability as "unchanging levels of a variable over time." When stability is 1 this means that a one unit difference at Time 1 predicts a 1 -unit difference at Time 2 , controlling for all other variables in the model. Stability greater than 1 would indicate that a one unit difference at Time 1 results in a greater than one unit difference at Time 2 . A stability less than 1 would indicate that a one unit difference at Time 1 results in a less than 1 unit difference at Time 2 .

\section{ANCOVA Model}

The ANCOVA model is the most general model discussed in Valente and MacKinnon (2017). This model examines the indirect effect of treatment $(X)$ on the outcome at Time 2 , through the mediator at Time 2 , controlling for the outcome and the mediator at Time 1. As readers may be more familiar with the ANCOVA model as described with the Time 2 variables as the outcomes, I'll describe how we translate to models with the difference scores as outcomes.

$$
\begin{aligned}
M_{2 i} & =i_{M_{2} A}+a_{M_{2} A} X_{i}+s_{M A}^{*} M_{1 i}+b_{M_{2} A} Y_{1 i}+e_{M_{2 i} A} \\
Y_{2 i} & =i_{Y_{2} A}+c_{Y_{2} A}^{\prime} X_{i}+s_{Y A}^{*} Y_{1 i}+b_{Y_{2} M_{1} A} M_{1 i}+b_{Y_{2} M_{2} A} M_{2 i}+e_{Y_{2 i A}}
\end{aligned}
$$

The mediator at Time 2 is predicted by treatment, the mediator at Time 1 , and the outcome at Time 1 . Note that $s_{M A}^{*}$ is the parameter which corresponds to the 
stability of the mediator. The outcome at Time 2 is predicted by treatment, the outcome at Time 1 , the mediator at Time 1 , and the mediator at Time 2 . The parameter $s_{Y A}^{*}$ is the stability of the outcome variable. To set the outcome as the difference between mediators for Equation 5.1, we subtract $M_{1 i}$ from each side and group terms.

$$
M_{2 i}-M_{1 i}=i_{M_{2} A}+a_{M_{2} A} X_{i}+\left(s_{M A}^{*}-1\right) M_{1 i}+b_{M_{2} A} Y_{1 i}+e_{M_{2 i} A}
$$

Note that now the coefficient for $M_{1 i}$ corresponds to the stability of the mediators minus 1. To set Equation 5.2 to include the difference between $Y$ s as the outcome and the difference between $M \mathrm{~s}$ as a predictor, we subtract $Y_{1 i}$ from both sides of the equation, and then we add and subtract $b_{Y_{2} M_{2} A} M_{1 i}$ to the right side of equation and group terms.

$Y_{2 i}-Y_{1 i}=i_{Y_{2} A}+c_{Y_{2} A}^{\prime} X_{i}+\left(s_{Y A}^{*}-1\right) Y_{1 i}+\left(b_{Y_{2} M_{1} A}+b_{Y_{2} M_{2} A}\right) M_{1 i}+b_{Y_{2} M_{2} A}\left(M_{2 i}-M_{1 i}\right)+e_{Y_{2 i A}}$

The coefficient for $Y_{1}$ is now the stability minus 1 . The coefficient for $M_{1}$ is now the sum of the relationship between $M_{1}$ and $Y_{2}$ and the relationship between $M_{2}$ and $Y_{2}$. The coefficient for the difference between the mediators is still the relationship between $Y_{2}$ and $M_{2}$, which is of direct interest as part of the indirect effect. I'll simplify the notation slightly to express the following equations for estimating the ANCOVA Model:

$$
\begin{aligned}
\Delta_{M_{i}} & =i_{M A}+s_{M A} M_{1 i}+a_{A} X_{i}+d_{A} Y_{1 i}+e_{M_{i} A} \\
\Delta_{Y_{i}} & =i_{Y A}+c_{A}^{\prime} X+b_{A} \Delta_{M_{i}}+f_{A} M_{1 i}+s_{Y A} Y_{1 i}+e_{Y_{i} A}
\end{aligned}
$$


In this equation the difference between $M_{1 i}$ and $M_{2 i}$ (i.e. $\Delta_{M_{i}}=M_{2 i}-M_{1 i}$ ) is predicted by $M_{1}, X$, and $Y_{1}$. The coefficient for $M_{1}, s_{M}$, is related to the stability of the mediator over time; it is the mediator stability minus $1\left(s_{M A}^{*}-1\right)$. The coefficient for $X, a_{A}$, is the estimated effect of the treatment on the mediator difference, which will be used in the estimate of the indirect effect. Similarly, the difference in the outcomes $\left(\Delta_{Y_{i}}=Y_{2 i}-Y_{1 i}\right)$ is predicted by the treatment $X$, the difference in the mediators, the mediator at Time 1 , and the outcome at Time 1 . The coefficient for $X, c_{A}^{\prime}$, is the estimate of the direct effect of the treatment on the outcome at Time 2 . The coefficient $b_{A}$ is an estimate of the effect of the mediator difference on the outcome at Time 2 , and this will be used to estimate the indirect effect. The coefficient $f_{A}$ estimates the degree to which $M_{1}$ predicts the difference in the outcomes above and beyond the difference in the mediators, this is a crosslag effect. Similar to $s_{M A}$, the coefficient $s_{Y A}$ is the outcome stability minus 1 .

In this model the estimate of the indirect effect of treatment on the outcome at Time 2 is $a_{A} b_{A}$. Each model has a slightly different interpretation of the indirect effect. For the ANCOVA model, it is interpreted as the effect of treatment on the outcome at Time 2 through treatment's effect on the mediator at Time 2 , controlling for the outcome and the mediator at Time 1. Valente and MacKinnon (2017) suggest that the ANCOVA model is optimal because in their simulations it has reasonable Type I Error and highest power across a variety of situations. Additionally, the estimate of the indirect effect was unbiased in their simulation study. They attribute this performance to the flexibility of the model, since it allows for differing levels of stability of the mediators (as estimated by $s_{M A}$ ) and the outcomes $\left(s_{Y A}\right)$ as well as the cross lag relationships $d_{A}$ and $f_{A}$. 


\section{Difference Score Model}

The Difference Score Model is most similar to the models I've discussed thus far in this dissertation. It focuses on "change" as the raw difference between scores at Time 1 and Time 2, rather than "controlling" for Time 1 measurements when predicting Time 2 measurements. The following equations can be used to estimate the Difference Score Model:

$$
\begin{aligned}
\Delta_{M_{i}} & =i_{M D}+a_{D} X_{i}+e_{M_{i} D} \\
\Delta_{Y_{i}} & =i_{Y D}+c_{D}^{\prime} X_{i}+b_{D} \Delta_{M_{i}}+e_{Y_{i} D}
\end{aligned}
$$

$\Delta_{M_{i}}$ and $\Delta_{Y_{i}}$ are defined the same as in the ANCOVA Model. Note that the model for $\Delta_{M_{i}}$ is the same as the ANCOVA model with some constraints: $s_{M A}$ and $d_{A}$ are constrained to be zero. Since $s_{M A}$ is the stability plus 1 , then the Difference Score Model assumes that stability is equal to one. Assuming that $d_{A}$ is zero means that we assume that $Y_{1}$ does not predict $M_{2}$ controlling for $M_{1}$ and $X$.

Similarly, the model for the difference in the outcomes is the same as the ANCOVA model with specific constraints: $s_{Y A}$ and $f_{A}$ are constrained to zero. This means the Difference Score Model is the same as the ANCOVA model, but where we've assumed that the stability of the outcome variables is 1 and there is no effect of $M_{1}$ on $Y_{2}$ after controlling for $\Delta_{M}$ and $X$.

In this model the estimate of the indirect effect of treatment on the difference in the outcomes is $a_{D} b_{D}$. The interpretation of the indirect effect in this models is the effect of treatment on change in the outcome through change in the mediator. Valente and MacKinnon (2017) preferred the ANCOVA model over the Difference 
Score Model, because they believed that assuming stability is 1 and cross lag effects are zero is too restrictive. Even with these restrictions, this model was unbiased in estimating the indirect effect, and had reasonable Type I Error, except when cross lag effects were high and/or stability was low. These cases are not surprising as they are the largest violations of the assumptions of the difference score model.

\section{Residualized Change Model}

The Residualized Change Model is another approach to assessing mediation in pre- post-test control group designs. Unlike the other models, the residualized change model takes two steps to estimate. First the Time 2 measurements must be predicted by their Time 1 measurements, then the residuals from these models are used in models to estimate the paths important for the indirect effect. Residualized change models are typically represented with equations where the residual is the outcome variable. I will show these models first, then show the equivalent model which uses difference scores. For clarity, I will use a lower case $r$ subscript for the residual outcome equations and a capital $R$ for the difference score outcome equations. For the first step we estimate:

$$
\begin{aligned}
M_{2 i} & =i_{M_{2} r}+s_{M r} M_{1 i}+e_{M_{2 i} r} \\
Y_{2 i} & =i_{Y_{2} r}+s_{Y r} Y_{1 i}+e_{Y_{2 i} r}
\end{aligned}
$$

By estimating Equations 5.8 and 5.9, we can get estimates of the errors in estimation $\hat{e}_{M_{2 i} r}$ and $\hat{e}_{Y_{2 i} r}$ these are what will be used in the models where we allows $X$ to predict the outcomes, as defined below: 


$$
\begin{aligned}
& \hat{e}_{M_{2 i} r}=i_{M r}+a_{r} X_{i}+e_{M_{i} r} \\
& \hat{e}_{Y_{2 i} r}=i_{Y r}+c_{r}^{\prime} X_{i}+b_{r} \hat{e}_{M_{2 i} r}+e_{Y_{i} r}
\end{aligned}
$$

In this model, we partial out all of the variance in $M_{2}$ and $Y_{2}$ which can be attributed to the Time 1 measures. In this way we take into account stability, like in the ANCOVA model. Similar to the difference score model there are no cross lag effects: $Y_{1}$ does not predict $M_{2}$ and $M_{1}$ does not predict $Y_{2}$. Reframing the model in terms of difference score makes the similarities and differences between this model and the other models more clear:

$$
\begin{aligned}
M_{2 i} & =i_{M_{2} R}+s_{M R} M_{1} i+1 \Delta_{M_{i}}+e_{M_{2 i} R} \\
Y_{2 i} & =i_{Y_{2} R}+s_{Y R} Y_{1 i}+1 \Delta_{Y_{i}}+e_{Y_{2 i} R} \\
\Delta_{M_{i}} & =i_{M R}+a_{R} X_{i}+e_{M_{i} R} \\
\Delta_{Y_{i}} & =i_{Y R}+c_{R}^{\prime} X+b_{R} \Delta_{M_{i}}+e_{Y_{i} R}
\end{aligned}
$$

From this representation it is clear that the Residualized Change Model bares certain similarities to both the ANCOVA and Difference Score Models. By estimating the parameters $s_{M R}$ and $s_{Y R}$ the Residualized Change Score Model allows stability to be estimated, rather than fixed. But there are not cross lag effects in these models. So the Residualized Change Score Model sits somewhere in between the Difference Score Model and the ANCOVA model.

In this model the estimate of the indirect effect of treatment on the outcome at Time 2 is $a_{R} b_{R}=a_{r} b_{r}$. We can interpret the indirect effect from this model as the 
effect of treatment on residual change in the outcome through residual change in the mediator, where residual change refers to variability in the posttest score that cannot be explained by the pretest score. Valente and MacKinnon (2017) suggest that the residual change model strikes a close second to the ANCOVA model as it is unbiased in estimating the indirect effect, has reasonable Type I Error, and high power (except when cross lags are present).

\section{Cross Sectional Model}

The cross section model is quite simple, it does not use the pre-measurements at all. Rather the model predicts $M_{2}$ using the treatment variable $X$ and $Y_{2}$ using $M_{2}$ and the treatment variable. I will not spend much time on this model, as Valente and MacKinnon (2017) found it does not perform well and is not truly taking advantage of the repeated-measures nature of the data. However for completeness this model is defined by the following regression equations:

$$
\begin{aligned}
M_{2 i} & =i_{M C}+a_{C} X_{i}+e_{M_{i} C} \\
Y_{2 i} & =i_{Y C}+c_{C}^{\prime} X_{i}+b_{C} M_{2 i}+e_{Y_{i} C}
\end{aligned}
$$

In this model the indirect effect is $a_{C} b_{C}$ and can be interpreted as the effect of treatment on the outcome at Time 2 through the mediator at Time 2 , not controlling for the Time 1 measurements. I will not discuss this model further as Valente and MacKinnon (2017) did not find it was a valid method for assessing mediation in prepost-test control group designs. 


\subsubsection{Difference Score Model and Conditional Process Model}

Equations 5.6 and 5.7, which define the Difference Score Model, are actually the same equations used in Chapter 3 to describe a first-stage conditional process model if we assume that the effect of $M$ on $Y$ is not moderated by instance (Equations 3.4 and 3.32 where $b_{2}$ is fixed to 0$)$. In Chapter 5 , I've used $X$ to denote experimental treatment because it is being used as the focal predictor in the mediation. It is the variable whose indirect effect we are estimating. In Chapter 3 , I used $W$ for the treatment because we are estimating the indirect effect of the repeated-measures factor (e.g., time) and allowing that indirect effect to be moderated by the experimental treatment.

So $a_{1}$ from Equation 3.4 and $a_{D}$ from Equation 5.6 are the same and estimate the degree to which treatment moderates the effect of the repeated-measure on the outcome and the degree to which treatment effects change in the mediator. Similarly, $b_{1}$ from Equation 3.32 and $b_{D}$ from Equation 5.7 are the same. They estimate the degree to which the mediator predicts the outcome. What is particularly interesting, is that the indirect effect of $X$ as defined by Valente and MacKinnon (2017) is the index of moderated mediation for the indirect effect of the repeated-measures factor as defined in Chapter 3. So these models are actually able to estimate both the conditional indirect effect of the repeated-measures factor (conditional on treatment), the indirect effect of treatment on change in the outcome, and the degree to which the treatment affects the indirect effect of the repeated-measures factor (the index of moderated mediation) is the same as the indirect effect of $X$.

Researchers can think about the model either way: the indirect effect of $X$ or the indirect effect of the repeated-measures factor. In fact, framing the model both ways 
might be particularly advantageous for theory. One particular advantage of thinking of the model as a conditional process model is that you get additional information than just thinking about the mediation model. When considering the repeated-measures factor, we can estimate the conditional indirect effect for individuals in the control condition. This gives us information about the expected change over time on $Y$ through $M$ without an intervention. Additionally, we can estimate the conditional indirect effect for individuals in the treatment condition. This tells us the expected change over time on $Y$ through $M$ for those who have been treated. The index of moderated mediation tells us the difference between these two. If we had just focused on the indirect effect $X$ on $Y$ through $M$ we would not learn about the expected change over time for the individual groups. However, if this is not of interest to the researcher, then framing the question with respect to the indirect effect of $X$ may make more sense.

\subsubsection{ANCOVA Model and Conditional Process Models}

Valente and MacKinnon (2017) show how the Difference Score Model is actually the ANCOVA model with specific assumptions. We know that the Difference Score Model is a model which can be used to assess whether treatment moderates the indirect effect of time on the outcome. So is the ANCOVA model a more general approach to assessing moderated mediation? What would the conditional indirect effects be if we used this more general model?

Think back to the original specification of the conditional process model in Equations 3.1 and 3.2. In the ANCOVA model, we freely estimate the relationship between 
$M_{2}$ and $M_{1}$ and $Y_{1}$. In the first-stage conditional process model, the relationship between $M_{1}$ and $M_{2}$ is constrained such that the stability is 1 , and the relationship between $Y_{1}$ and $M_{2}$ is constrained to be zero. Similarly, when we use Equation 3.2 for the model of $Y$ we constrain the relationship between $Y_{1}$ and $Y_{2}$ such that the stability is 1 and the relationship between $M_{1}$ and $Y_{2}$ is fixed to zero. In the ANCOVA model, these are freely estimated. Next I discuss instances when these assumptions may and may not be reasonable.

One major difference between the conditional process model discussed in Chapter 3 and the ANCOVA model is the assumption about stability. By assuming that stability for $M$ and $Y$ is 1 in the conditional process model, we are assuming that a one unit difference at Time 1 predicts a 1 unit difference at Time 2, on average. There may be cases when we expect stability to be 1. For example, with careful choice of outcome and mediator measurement instruments, taking into account variability, expected change, and providing a wide enough range in response categories, many within-subjects experimental designs can avoid floor or ceiling effects. However, if there are floor or ceiling effects, or any concern about regression to the mean then assuming that stability is 1 may be unreasonable. The researcher might consider estimating the stability rather than assuming it. In this case the residualized change score or ANCOVA model would be useful to estimating this type of model. The choice between the residualized change score and the ANCOVA model would be driven by whether the researcher wants to include cross-lag paths.

The other assumption that is different between ANCOVA and the conditional process model is the cross-lag paths. In the ANCOVA model, $M_{1}$ is allowed to predict $Y_{2}$ above and beyond $Y_{1}$ and $M_{2}$. Additionally, $Y_{1}$ is allowed to predict $M_{2}$. 
In longitudinal studies this will often make sense, as psychological processes are often continuous though we're measuring them at fixed points in time. However, in cases where 1 and 2 do not necessarily correspond to time but to some other instance, it may be less reasonable to assume that these cross lag paths exist. For example, in Chapter 4 we considered an example where the repeated-measurements corresponded to two different computer science courses. For half of the participants, $M_{2}$ is happening "before" $Y_{1}$ (since 2 corresponded to the group work class and 1 corresponded to the individual work class). Psychological theory would not suggest there is any reason that interest in the individual work class would predict perceptions of communal goals in the group work class above and beyond communal goals in the individual work class. A similar argument can be made against allowing communal goals in the group work class to predict interest in the individual work class above and beyond interest in the individual work class and communal goals in the group work class. Often, the cross lag paths make sense in a longitudinal framework. However, for the more general two-instance repeated-measures design, assuming these cross lag paths are zero may be more in line with the theoretical framework being tested. Additionally, Valente and MacKinnon (2017) found that the Difference Score Model was unbiased in estimating the indirect effect even when the cross-lag paths existed. This suggests that the conditional process model should be unbiased in estimating the index of moderated mediation even when there are cross-lagged paths. But the Difference Score Model had lower power in comparison to the ANCOVA approach, so we might expect the same from the conditional process model. Therefore in instances when theory suggests there are cross-lag paths, it seems the ANCOVA model would be more advantageous. 
One issue with the simulations in Valente and MacKinnon (2017) is that they did not simulate any conditions where the Difference Score Model was the true generating model (i.e., stability was 1). They only generated data with stability of 0.3 and 0.7 . It would be worthwhile to investigate if the Difference Score Model has higher power than the ANCOVA model to detect the indirect effect (or the index of moderated mediation for time) when stability is 1 . I expect this would be the case, because the Difference Score Model would be more parsimonious. This would also suggest that the conditional process model would be preferred to the ANCOVA model when there are no cross-lagged paths and stability is 1 .

\subsection{Multilevel Conditional Process Models}

Multilevel models are used throughout psychology, education, and other scientific fields to include dependencies among observations in a statistical model. These dependencies are taken into account by defining multiple levels of analysis (e.g., a model of the responses from an individual and a model of individuals). This allows responses which are nested within an individual to be similar, as they include a unique set of parameters specific to the individual from which the responses are generated. One major advantage of multilevel models is that they allow some parameters in the model of the responses to be individual-specific.

The two-instance repeated-measures design can be a useful starting place for discussing conditional process analysis in repeated-measures designs as the connection back to familiar approaches to conditional process analysis is clear. However, with one observation for each individual in each instance, there are very little data. This means that some of the distinct advantages of multilevel models are not available in 
such a simple design. For example, researchers may be interested in understanding variability within an individual which is not estimable in the two-instance repeatedmeasures conditional process models described in this dissertation. However, if we have data with multiple replicates per instance, we could use multilevel models to estimate within-individual variability. In this section, I describe a subset of multilevel conditional process models, where the mediation operates among variables measured at Level 1 and the moderator is a Level 2 variable. I show how this model is related to the models presented in Chapter 3.

I'll begin with the 1-1-1 multilevel mediation model and then build in the moderation component. As a slight change in notation, the instance under which the measurement was made will no longer be represented as a subscript, but rather a dichotomous predictor $X=0,1$ will represent instance. This means that the measure $M_{i k}$ refers to the measurement on the $i$ th individual on the $k$ th replicate, where $k=1, \ldots, K$ and $K$ is the total number of measurements made on individual $i$ rather than the number in a specific condition. The variable $X_{i k}$ will denote the condition for the $k$ th replicate for individual $i$. Similarly $Y_{i k}$ is the outcome measure for the $k$ th replicate for the $i$ th individual. In a two instance repeated measures designs $K=2$.

\subsubsection{1-1-1 Mediation}

The variables involved should be individual mean centered in the Level 1 equations and the individual means entered into the Level 2 equations. This eliminates the confounding between Level 1 and Level 2 mediation effects (Zhang, Zyphur, \& Preacher, 2009). We will denote the means for individual $i$ as $\bar{X}_{i}=1 / K \sum_{k=1}^{K} X_{i k}$, 
and $\bar{M}_{i .}=1 / K \sum_{k=1}^{K} M_{i k}$. The individual centered variables will be denoted with a $c$ superscript. For example, $X_{i k}^{c}=X_{i k}-\bar{X}_{i \text {. }}$

The first model we consider is the model for the total effect. This is the overall effect of $X$, instance, on the outcome.

$$
\begin{aligned}
Y_{i k}= & c_{0 i}+c X_{i k}^{c}+e_{Y_{i k}} \\
& e_{Y_{i k}} \sim N\left(0, \sigma_{Y}^{2} I\right) \\
c_{0 i}= & \gamma_{00}+\gamma_{01} \bar{X}_{i}+u_{c_{0 i}} \\
& u_{c_{0 i}} \sim N\left(0, \tau_{c_{0}}^{2}\right)
\end{aligned}
$$

Here the outcome for individual $i$ at replicate $k, Y_{i k}$, is a linear function of a person specific intercept $c_{0 i}$ and the condition for person $i$ in replicate $k, X_{i k}$, and a person and replicate specific error term $e_{Y_{i k}}$. The regression weight for the instance $c$ is the total effect, as this is the expected change in $Y$ given a single unit change in $X$, which when $X$ is coded as a 0,1 dichotomous variable, means the expected difference in $Y$ from one condition to another. $I$ is a $K \times K$ identity matrix. The errors in this model are assumed to be independent and identically distributed with a mean of zero and a common variance $\sigma_{Y}^{2}$.

The individual-specific intercept has a fairly basic model which consists of an intercept, the individual's average $X$, and an error term. Each individual's intercept can be described as a random sample from the normal distribution with mean $\gamma_{00}+$ $\gamma_{01} \bar{X}_{i}$. and variance $\tau_{c_{0}}^{2}$. The parameter $c_{0 i}$ is a combination of the population mean $\gamma_{00}+\gamma_{01} \bar{X}_{i}$. and an individual deviation from that mean $u_{c_{0 i}}$. More complicated 
models can be used where other individual level predictors of $c_{0 i}$ can be included. I will not describe these more complicated models here.

The model for the effect of condition on the mediator is very similar to the model for the total effect except the outcome is the mediator.

$$
\begin{aligned}
M_{i k}= & a_{0 i}+a X_{i k}^{c}+e_{M_{i k}} \\
& e_{M_{i k}} \sim N\left(0, \sigma_{M}^{2} I\right) \\
a_{0 i}= & \alpha_{00}+\alpha_{01} \bar{X}_{i}+u_{a_{0 i}} \\
& u_{a_{0 i}} \sim N\left(0, \tau_{a_{0}}^{2}\right)
\end{aligned}
$$

Similar to the previous model mediator for individual $i$ at replicate $k, M_{i k}$, is a linear function of a person specific intercept $a_{0 i}$ and the instance for person $i$ in replicate $k, X_{i k}^{c}$, and a person and replicate specific error term $e_{M_{i k}}$. The regression weight for the instance $a$ represents the effect of instance on the mediator. The errors in this model are assumed to be independent and identically distributed with a mean of zero and a common variance $\sigma_{M}^{2}$. In the Level 2 model for the individual specific intercept, each individual's intercept $a_{0 i}$ is the combination of the population mean $\alpha_{00}+\alpha_{01} \bar{X}_{i}$. and an individual deviation from that mean $u_{a_{0 i}}$.

The model for the outcome $Y$ is

$$
\begin{aligned}
Y_{i k}= & c_{0 i}^{\prime}+c^{\prime} X_{i k}^{c}+b M_{i k}^{c}+e_{Y_{i k}^{\prime}} \\
& e_{Y_{i k}^{\prime}} \sim N\left(0, \sigma_{Y^{\prime}}^{2} I\right) \\
c_{0 i}^{\prime}= & \gamma_{00}^{\prime}+\gamma_{01}^{\prime} \bar{X}_{i .}+\beta_{01} \bar{M}_{i .}+u_{c_{0 i}^{\prime}} \\
& u_{c_{0 i}^{\prime}} \sim N\left(0, \tau_{c_{0}^{\prime}}^{2}\right) .
\end{aligned}
$$


Here we are predicting the outcome for individual $i$ in replicate $k$ from the condition $X_{i k}$ and the mediator $M_{i k}$. Again, the intercept is individual-specific, which allows responses from the same individual to be more similar to each other but fixes the relationships between $Y, X$, and $M$ to be the same across all individuals. The coefficient for instance, $c^{\prime}$ represents the direct effect (i.e. the effect of instance on the outcome holding the mediator constant). The coefficient for the mediator $b$ represents the relationship between the mediator and the outcome holding instance constant. Similar to before, the model for the individual specific intercept includes a population mean $\gamma_{00}^{\prime}+\gamma_{01}^{\prime} \bar{X}_{i}+\beta_{01} \bar{M}_{i}$. and an individual deviation from that mean $u_{c_{0 i}^{\prime}}$.

This outlines the multilevel models needed to evaluate an a multilevel mediation model using fixed slopes. The effects of particular interest in a mediation model are the total effect $c$, the direct effect $c^{\prime}$ and the indirect effect, which is the product of $a$ and $b$. For these designs, I focus on the intra-individual (Level 1) indirect effect. This is an estimate of how much change in $X$ within an individual predicts change in $Y$ within an individual through change in $M$ within an individual. By contrast there is another indirect effect which could potentially be of interest. This is the Level-2 effect where variance in $\bar{X}_{i}$. across individuals, results in change in the $Y_{i k}$ through change in $\bar{M}_{i}$. This indirect effect is captured by $\alpha_{01} \beta_{01}$. This effect is discussed more in Zhang et al. (2009).

If every individual experiences the same number or proportion of replicates in each instance then $\bar{X}_{i}$. will be constant across $i$. Therefore there will be no variance in $\bar{X}_{i}$. to cause variance in $c_{0 i}^{\prime}$. When every individual experiences the same number or proportion of the instances then this effect will always be zero. Interest in the Level 2 indirect effect originally arose when discussing nested data (e.g., individuals nested 
within groups could have differing group means) and so correcting for the group means and disaggregating the Level 1 and Level 2 indirect effects is very important. In the repeated-measures case this can arise if for some reason the replicates are not balanced across individuals, for example if some individuals have $3 / 4$ of their replicates from one instance and some individuals have $1 / 2$ of their replicates from each instance. A particularly important case where this might arise is if the original design is balanced, but missing data results in some individuals having a different proportion of replicates from each instance.

\subsubsection{1-1-1 Conditional Process Models}

Next I'll discuss the multilevel conditional process model based on the equations above. Essentially, each effect in the mediation model can be moderated by a Level 2 predictor. This is done by allowing there to be an interaction between the Level 1 predictor and the Level 2 predictor. Now including a Level 2 predictor $W_{i}$ we can reformulate the model for $M_{i k}$ and the model for $Y_{i k}$. Note that the moderator only has the subscript $i$ because it is specific to the individual, but does not vary across instances $k$. The equations for the mediator is now

$$
\begin{aligned}
M_{i k}= & a_{0 i}+\left(a_{1}+a_{2} W_{i}\right) X_{i k}^{c}+e_{M_{i k}} \\
& e_{M_{i k}} \sim N\left(0, \sigma_{M}^{2} I\right) \\
a_{0 i}= & \alpha_{00}+\alpha_{01} \bar{X}_{i}+\alpha_{02} W_{i}+u_{a_{0 i}} \\
& u_{a_{0 i}} \sim N\left(0, \tau_{a_{0}}^{2}\right)
\end{aligned}
$$

Here in the Level 1 equation we allow the the effect of $X_{i k}$ to be a linear function of the moderator $a_{1}+a_{2} W_{i}$. Additionally, we add $W_{i}$ alone to the Level 2 equation. 
To allow the effect of $M$ on $Y$ and the direct effect to be moderated, each of these effects is specified as a linear function of $W$. The equation for $Y_{i k}$ is

$$
\begin{aligned}
Y_{i k}= & c_{0 i}^{\prime}+\left(c_{1}^{\prime}+c_{2}^{\prime} W_{i}\right) X_{i k}^{c}+\left(b_{1}+b_{2} W_{i}\right) M_{i k}^{c}+e_{Y_{i k}^{\prime}} \\
& e_{Y_{i k}^{\prime}} \sim N\left(0, \sigma_{Y^{\prime}}^{2} I\right) \\
c_{0 i}^{\prime}= & \gamma_{00}^{\prime}+\gamma_{01}^{\prime} \bar{X}_{i \cdot}+\beta_{01} \bar{M}_{i .}+\beta_{02} W_{i}+u_{c_{0 i}^{\prime}} \\
& u_{c_{0 i}^{\prime}} \sim N\left(0, \tau_{c_{0}^{\prime}}^{2}\right) .
\end{aligned}
$$

Now the conditional effect of $M$ on $Y$ is $b_{1}+b_{2} W_{i}$, and the conditional direct effect is $c_{1}^{\prime}+c_{2}^{\prime} W_{i}$. The conditional indirect effect would be $\left(a_{1}+a_{2} W_{i}\right)\left(b_{1}+b_{2} W_{i}\right)$. A simpler model could be fit where the effect of instance on the mediator or the effect of the mediator on the outcome is not moderated.

\subsubsection{Connection to Regression Based Method}

Though the way the equations are expressed for the multilevel models is different than the regression model, in the special case of a balanced two-instance repeatedmeasure design, the regression model and the the multilevel model are actually equivalent and provide the same point estimates for each parameter and very similar standard error estimates. The standard error estimates differ somewhat because of the estimation method used for multilevel models, which is more complex than ordinary least squares.

To see the similarities between the two models, I'll rewrite the equations for the multilevel model as a single equation for each outcome, rather than broken into the 
Level 1 and Level 2 components.

$$
\begin{aligned}
M_{i k}= & \alpha_{00}+\alpha_{01} \bar{X}_{i .}+\alpha_{02} W_{i}+u_{a_{0 i}}+\left(a_{1}+a_{2} W_{i}\right) X_{i k}^{c}+e_{M_{i k}} \\
Y_{i k}= & \gamma_{00}^{\prime}+\gamma_{01}^{\prime} \bar{X}_{i \cdot}+\beta_{01} \bar{M}_{i \cdot}+\beta_{02} W_{i}+u_{c_{0 i}^{\prime}}+\left(c_{1}^{\prime}+c_{2}^{\prime} W_{i}\right) X_{i k}^{c} \\
& +\left(b_{1}+b_{2} W_{i}\right) M_{i k}^{c}+e_{Y_{i k}^{\prime}}
\end{aligned}
$$

Now, I'll make two equations for $M_{i k}$ and $Y_{i k}$ where we plug in $X_{i k}=0$ and $X_{i k}=1$ for each equation. Again, remember that in the balanced two-instance repeated-measure design the average of $X_{i k}$ is equal across individuals, so I'll take that part out of the equation. I also group terms to aid comparison.

$$
\begin{aligned}
& \left(M_{i k} \mid X_{i k}=0\right)=\alpha_{00}+\alpha_{02} W_{i}+u_{a_{0 i}}+e_{M_{i k}} \\
& \left(M_{i k} \mid X_{i k}=1\right)=\left(\alpha_{00}+a_{1}\right)+\left(\alpha_{02}+a_{2}\right) W_{i}+u_{a_{0 i}}+e_{M_{i k}}
\end{aligned}
$$

Compare these equations to Equations 3.1 and 3.2.

$$
\begin{aligned}
& M_{1 i}=a_{10}+a_{11} W_{i}+e_{M_{1 i}} \\
& M_{2 i}=a_{20}+a_{21} W_{i}+e_{M_{2 i}}
\end{aligned}
$$

It is easy to see how these two sets of equations correspond. These equation are equivalent such that: 


$$
\begin{aligned}
a_{10} & =\alpha_{00} \\
a_{20} & =\alpha_{00}+a_{1} \\
a_{11} & =\alpha_{02} \\
a_{21} & =\alpha_{02}+a_{2} \\
e_{M_{1 i}} & =u_{a_{0 i}}+e_{M_{i k}} \\
e_{M_{2 i}} & =u_{a_{0 i}}+e_{M_{i k}}
\end{aligned}
$$

And from this it is clear to see that the parameters which are used to estimate the conditional indirect effect are the same in each type of model. The effect of instance on the mediator when the moderator is zero is $a_{10}-a_{20}=a_{1}$, and the degree to which $W$ moderates the effect of instance on the mediator is $a_{11}-a_{21}=a_{2}$.

Something similar occurs with the model of the outcomes; however the model from 3.11 and 3.12 are not exactly the same as the multilevel model. So I will also discuss the assumptions which make them equivalent. The multilevel model equations are

$$
\begin{aligned}
& \left(Y_{i k} \mid X_{i k}=0\right)=\gamma_{00}^{\prime}+\beta_{01} \bar{M}_{i .}+\beta_{02} W_{i}+u_{c_{0 i}^{\prime}}+\left(b_{1}+b_{2} W_{i}\right) M_{i k}^{c}+e_{Y_{i k}^{\prime}} \\
& \left(Y_{i k} \mid X_{i k}=1\right)=\left(\gamma_{00}^{\prime}+c_{1}^{\prime}\right)+\beta_{01} \bar{M}_{i}+\left(\beta_{02}+c_{2}^{\prime}\right) W_{i}+u_{c_{0 i}^{\prime}}+\left(b_{1}+b_{2} W_{i}\right) M_{i k}^{c}+e_{Y_{i k}^{\prime}}
\end{aligned}
$$

and the regression based equations are

$$
\begin{aligned}
& Y_{1 i}=c_{1}^{\prime *}+\left(b_{11}+b_{13} W_{i}\right) M_{1 i}+b_{12} W_{i}+e_{Y_{1 i}} \\
& Y_{2 i}=c_{2}^{*}+\left(b_{21}+b_{23} W_{i}\right) M_{2 i}+b_{22} W_{i}+e_{Y_{2 i}}
\end{aligned}
$$


The first thing to note would be that the multilevel equations involve a term for $\bar{M}_{i}$. whereas the regression based equations do not. This is because the regression based equations are used for estimating within-person (or Level 1) indirect effects, and $\beta_{01}$ is not needed for estimating Level 1 indirect effects. Next, the regression based equations allow for the interaction between instance and the mediator, allowing $b_{11} \neq b_{21}$ and $b_{13} \neq b_{23}$. The multilevel model does not allow for this, though it could. Instead if we assume that $b_{11}=b_{21}$ and $b_{13}=b_{23}$ then the following equivalences hold:

$$
\begin{aligned}
c_{1}^{\prime *} & =\gamma_{00}^{\prime} \\
c_{2}^{\prime *} & =\gamma_{00}^{\prime}+c_{1}^{\prime} \\
b_{11} & =b_{21}=b_{1} \\
b_{13} & =b_{23}=b_{2} \\
e_{Y_{1 i}^{\prime}} & =u_{c_{0 i}^{\prime}}+e_{M_{i k}} \\
e_{Y_{2 i}^{\prime}} & =u_{c_{0 i}^{\prime}}+e_{M_{i k}}
\end{aligned}
$$

This shows under what assumptions the regression based approach would be equivalent to the multilevel approach. The multilevel model presented assumes that there is no interaction between instance and the mediator in the model for $Y$. The regression based approach can be adjusted to accomodate this assumption as discussed in Section 3.4.

\subsubsection{Advantages and Disadvantages of MLM approaches}

Multilevel modeling is a more general approach to conditional process analysis than the very specific approach described in this dissertation which uses difference 
scores and linear regression. The two approaches will produce the same answers under certain assumptions, but multilevel models can be generalized far beyond the twoinstance repeated-measures case. Multilevel models would be able to accommodate multiple replicates for each individual in each instance. They can accommodate more than two instances, or some continuous version of $X$ rather than just a dichotomous $X$. The moderator could be Level 1 or Level 2. All models I discussed in this section had fixed slopes and random intercepts, but the slopes could also be specified as random. This adds additional complexity to the model that can be useful when theory suggests that the slopes may vary by individual, rather than being the same for each individual. Including random slopes also lends better to the interpretation of the model as one of intra-individual change, since the change is measured at the individual level using the individual random coefficients. Literature which discusses more general applications of conditional process models with multilevel models include Bauer and Curran (2005) and Rockwood (2017).

So why use the regression based method? One of the purposes of this dissertation is to connect statistical approaches. Knowing that a method which uses OLS regression with difference scores is the same as a multilevel approach under certain conditions is informative. Additionally, many researchers are familiar with linear regression but are less so with multilevel models. My hope in introducing the connections between these two methods is that the regression based method might aid researchers in taking a step toward multilevel models and, eventually, make the leap completely. Another reason to use the regression method is that a closed form solution is always available. Multilevel methods use estimation methods that do not guarantee convergence. When the variance of one of the random intercepts is very low (near zero), some multilevel 
estimation methods may not converge on an answer. Using the regression based method, convergence is always guaranteed.

\subsection{Structural Equation Approaches to Conditional Process Analysis}

There are a variety of methods that use structural equation modeling to assess mediation with repeated measures data. In this section I discuss a few of these approaches and some of the general advantages of using structural equation modeling.

The first and potentially most simple structural equation modeling approach would be estimating the initial models outlined in Chapter 3 (Equations 3.1, 3.2, $3.11,3.11)$ while allowing for correlated residuals. The mediation approach to this was discussed in Judd et al. (2001). The extension to moderated mediation is not complex, as you would just include a new variable $W$ and allow the appropriate products. This method, however, does not scale up well to more than two instances. One advantage this method has is that you can have latent variables instead of observed variables, so if the mediator or the outcome is measured using a multiple item scale, this can be properly taken into account in the statistical model. This is important because measurement error can bias the estimate of the indirect effect (VanderWeele, Valeri, \& Ogburn, 2012).

Two models I will discuss together are the latent difference model and the latent growth curve model. The latent difference method for assessing mediation is discussed in MacKinnon (2008), Selig and Preacher (2009), and Wu, Selig, and Little (2013), and Preacher (2015) in terms of mediation. The latent growth approach to mediation is discussed in Cheong (2011), Cheong et al. (2003), Cole and Maxwell (2003), and Selig and Preacher (2009). However, the generalization to moderated mediation has yet 
to be discussed for either of these models. The latent difference score model has the advantage of being able to treat the difference score as latent rather than observed. The latent growth curve model allows for more than two time points to be modeled, easily generalizing beyond the two-instance repeated-measures case, especially when instance is time. Generalization of these models to conditional process analysis is beyond the scope of this dissertation but would be a fruitful area of future research. I expect that with simple cases like the two-instance repeated-measures design, the results of the regression based approach and these more complex structural equation models would be the same. However, like multilevel modeling, these SEM methods scale up beyond the two-instance repeated-measures design much more easily than the difference score regression approach.

A very popular method for longitudinal mediation is cross-lagged panel models. This model is essentially a generalization of the ANCOVA approach described in section 5.1.1, where more than two time points can be assessed. These models have been described by Preacher and Selig (2012), Maxwell and Cole (2007), Mitchell and Maxwell (2013), and Selig and Preacher (2009). Similar to the latent difference and latent grown models, these models have not yet been discussed in the context of moderated mediation. Just like in the ANCOVA model, the two-instance repeatedmeasures design case the cross-lagged panel design is equivalent under certain assumptions (i.e., no cross-lag effects and stability fixed to 1). The cross-lag panel model has a few advantages over the difference score model in that it can accommodate latent variables, and more than two time points easily. However, there have been some issues identified with the cross-lag panel mediation models, in that they do 
not estimate longitudinal mediation effects without bias (Maxwell, Cole, \& Mitchell, 2011).

\subsection{Summary}

There are a variety of alternative approaches to assessing moderated mediation in two-instance repeated-measures designs. Some alternatives involve thinking about the moderator as the causal variable of interest and ignoring the effect of instance (Valente \& MacKinnon, 2017). This may be useful for researchers with 2(within) x 2(between) designs, who are not overly interested in the effect of instance. However, researchers are often interested in both the effect of time and the effect of the moderator, which means that thinking about the problem as a conditional process model rather than just mediation, can provide additional detail which is ignored when just considering mediation.

Additionally, there are multilevel conditional process models that are equivalent to the regression based approach described in Chapter 3 in simple designs such as the two-instance repeated measures design. Multilevel mediation can also be used for more expansive design such as two-instance repeated-measures designs with multiple replicates per condition, more than two-instances, continuous independent variables, and much more. Multilevel models also allow for random slopes and more complex models. Hopefully, individuals who learn about the regression based model will become curious about some of the advantages of multilevel models and explore these possibilities even more. 
There are a variety of structural equation modeling approaches to mediation in repeated-measures data (most of which are focused on longitudinal data). The correlated residual model, latent difference score model, growth curve model, and crosslag panel model have all been used to explore mediation in repeated-measures data. However, to date these methods have not been used for moderated mediation. Future research should explore the expansion of these models to moderated mediation and how these models perform in detecting moderated mediation.

This chapter has explored a variety of alternative methods to the regression based method I have focused on throughout the dissertation. This chapter has shown when many of these alternatives are equivalent and what some potential advantages and disadvantages of these alternatives might be. Next, I conclude this dissertation by discussing common themes, limitations, and future directions. 


\section{Chapter 6: Discussion}

Previous research on conditional process analysis has primarily been limited in focus to between-subjects designs. However, repeated-measures designs are very common in psychology and other social sciences. This dissertation has unified the research on mediation and moderation analysis in two-instance repeated measures design to describe how to conduct conditional process analysis in these designs. This development allows researchers who are investigating questions of conditional processes to use repeated-measures designs. These design can increase power and the intra-individual interpretation of indirect effects.

Additionally, researchers in areas that frequently use repeated-measures designs may not have previously been able to investigate questions of moderated mediation. My aim with this dissertation was to open the doors for researchers in these areas so they can begin using these types of analyses. Hopefully this will encourage new types of hypotheses within these areas, expanding their theoretical breadth.

I focus on the two-instance repeated-measure design because it is one of the sim-

plest repeated-measures designs and bares a close resemblance to a between-subjects experiment. Mediation analysis is frequently used when data are collected in betweensubjects experiments. My hope is that researchers familiar with mediation analysis 
will see the connection between the methods proposed in this dissertation and mediation analysis methods for between subjects designs. Additionally, by focusing on the two-instance repeated-measures design, linear regression (a method many researchers are familiar with) can still be used, eliminating the need to take the leap to multilevel modeling. However, as described in Chapter 5, taking this leap opens many doors to creating interesting and complex moderated mediation models. Typically, we think of linear regression as only appropriate for data where the cases are independent. This dissertation shows how we can use linear regression in combination with difference scores to properly account for the dependencies among the observations of individuals in a valid way. This should make it clear to researchers new to repeated-measures designs the importance of taking into account dependencies, without requiring a whole new skill such as multilevel modeling.

Overall, the aim of this dissertation was to describe how to conduct conditional process analysis in two-instance repeated-measures designs. Additionally, I provided examples so that it is clear what types of data these methods can be applied to and how to interpret the results of such an analysis. I described some alternative interpretations and alternative approaches to conditional process analysis in the two-instance repeated measures design. In this Chapter I will conclude with a few limitations, both of the analytical approach and of the design which I have focused on. I will also discuss a variety of extensions and future directions that may be of potential interest.

\subsection{Limitations}

The limitations of the methods described in this dissertation can be divided into limitations of the analytical approach and limitations of the design. This section 
describes some of these limitations, though there are undoubtedly more. The purpose of these descriptions is to clarify under what conditions the proposed methods are optimal and when might researchers consider using a different method or a different design.

\subsubsection{Analytical Approach}

One of the largest limitations of the analytical approach proposed in this dissertation is the difficulty in scaling it up. For example, if instead of one observation per instance for each participant there were multiple observations per instance, the researcher would have to average over those observations to conduct the analysis as described in this paper. By averaging across the multiple observations, information about within person response variability is lost. This information could easily be captured in a multilevel model. However, the regression based approach described in this dissertation is not able to account for that variability. Another way the design does not scale up easily is with additional conditions. As will be described later in the future directions sections, there are ways to define contrasts so that moderated mediation could be evaluated for more than two conditions. However approaches such as multilevel modeling and structural equation modeling require the analyst to do less work up front in defining contrasts of interest and finding the orthogonal contrasts to estimate the models. Multilevel modeling and SEM tools make this process very easy. As they currently stand, the tools for the regression based approach do not make this very easy at all.

Previous research has criticized the use of difference scores as measures of change, suggesting that they are insensitive to artifacts such as regression to the mean, ceiling 
effects, and floor effects (Bonate, 2000; Cronbach \& Furby, 1970; Lord, 1963; Twisk $\&$ Proper, 2004). However, many have argued that the merits of difference scores are greater than some researchers credit them (Thomas \& Zumbo, 2012; Rogosa, 1995; Zumbo, 1999). As discussed in Chapter 5, artifacts such as regression toward the mean are also an issue with the analysis described in this dissertation. Regression to the mean influences the stability of the mediator and outcome variable across repeated measurements. The Difference Score Model assumes that a one unit difference on the mediator at Time 1 results in a one unit difference in the mediator at Time 2. If this assumption is not valid then other methods such as the ANCOVA or Residualized Change Model would to a better job of accounting for this inconsistency. However, based on the results of Valente and MacKinnon (2017), even in the presence of regression toward the mean, the Difference Score Model is unbiased in estimating the index of moderated mediation, though it does have lower power than the other methods. Regression to the mean is particularly prevalent when measuring individuals longitudinally, but remember that the two-instance repeated-measures design covers designs beyond pre-post designs. A two-condition within-subjects experiment might be a situation in which the assumption that the stability of the mediator and outcome are both one would make sense, especially when order is counterbalanced. So the more appropriate procedure for these types of designs would be a the Difference Score Model described in this dissertation. However, especially in the case of a longitudinal model, researchers should consider whether they want to make the assumption about stability or estimate it and choose the type of model which is most appropriate based on what assumptions they are willing to make. 
Much of the research done in psychology relies on measurement of latent constructs. These are constructs that we cannot see nor directly measure but, rather, constructs that we have to indirectly measure. Typically in psychology we ask participants to respond to a variety of questions which all act as indicators for a latent construct. Latent variable modeling techniques can be used to simultaneously estimate a measurement model and a structural model to account for the fact that we are not directly observing the variables we are interested in. The method described in this paper does not allow for a measurement model. This method treats the measurements as direct observations rather than indicators of some latent variable. As described in Chapter 5, expanding latent difference models and latent growth models to conditional process models would be advantageous. This expansion would allow us to benefit from the measurement model with the moderated mediation structural component all in one analytical approach.

\subsubsection{Design}

One of the core assumptions mediation is the causal nature of the relationship among the variables. As mentioned very early in this dissertation, mediation assumes that you have the causal order of the variables correctly specified. In the situation of a two-condition within-subjects experiment, the design gives credence to the assumption that the repeated-measures variable is causing change in the mediator and the outcome. This still leaves the mediator to outcome relationship, which must be assumed to be properly specified. This is a common problem in a variety of designs, including between-subjects experiments. However, some two-instance repeated-measures designs conflate time and the variable which is meant to be the 
causal variable of interest. For example, consider the study by Lasselin et al. (2016). They measured individuals before a treatment and then after a treatment, and the change in the mediators and outcome was attributed to the treatment. However, without a control condition where individuals did not undergo treatment over the same period it is difficult to know if the change over time would have occurred if the treatment had not been administered. The extension to conditional process analysis improves the general mediation approach, since now we can include a variable which codes experimental vs. control condition as a moderator, whereas before we could not. But designs that observe change over time and have some other moderator than an experimental condition (e.g., baseline inflammation) will conflate change over time with the effect of the independent variable. Better designs would include a treatment and no treatment condition to evaluate the natural effect of time.

By measuring individuals only twice, the model of change is fixed to be a linear model regardless of the analytical technique used. There may be many cases where change is expected to occur non-linearly and would thus require more points of repeated-measurement. For example, imagine you are interested in understanding the effect of a temporary pain reliever (e.g., ibuprofen). You might ask each participant to come to the lab at and received a different dose of the drug each time. Doses could range from $0 \mathrm{mg}$ to $800 \mathrm{mg}$ in $100 \mathrm{mg}$ increments. This would mean that each participant is measured nine times. With nine repeated measurements, there are a variety of models of change that could be fit to an individual's data. For example, you might expect a curvilinear relationship such that the decrease in pain with each 100mg increase slowly flattens out. This type of the theory could not be examined 
with the two-instance repeated-measures design, because we're only investigating individuals at two-instances. This means that the difference between the two instances is inherently treated as linear change, and in order to examine more complex models of change, a more complex design is required.

\subsection{Future Directions}

There are a variety of future directions and extensions that follow from the research presented in this dissertation. I've tried to note these as they've come up in my discussion, but in this section I discuss these all together as well as some considerations that should be taken into account when pursuing these directions and extensions.

One future direction would be expanding the model discussed in Chapter 3 to ones with multiple mediators in parallel or in serial. Montoya and Hayes (2017) generalized the two-instance repeated-measures mediation model to multiple mediators, and Hayes (2015) generalized the index of moderated mediation to multiple mediators models in between subjects designs. The principles from Chapter 3 can be applied to any parallel or serial mediator model, where any path that is moderated would be specified as a linear function of the moderator. The indirect effect is still the product

of the paths leading from $X$ to $Y$. In particular, in the case of serial mediation, the serial indirect effect is the product of three or more paths depending on how many mediators there are in serial. If each of these paths are moderated, this means that the conditional serial indirect effect could be a high degree polynomial function of the moderator. This could lead to very interesting patterns of significance of the indirect effect across the range of the moderator. In general, the extension to multiple 
mediator models would not be difficult. Perhaps the greatest difficulty would be in implementing such an analysis in a statistical tool.

Just as there could be multiple mediators, there could also be multiple moderators in the conditional process model for two-instance repeated measures designs. As described in Hayes (2018a) and Montoya (in press), multiple moderation can occur in two ways: multiplicative moderation, where the moderators interact with each other, and additive moderation, where the moderators do not interact. Additive moderation would be the simpler case, and if there were additional moderators in the conditional process models described in Chapter 3, the conditional effects would be linear functions of the moderators. If the moderators interact with each other, then this interaction term would need to be included in the conditional effects. This could lead to very complex functions defining the conditional indirect effect, especially if multiple paths in the mediation model are moderated by multiple moderators. Hayes (2018a) describes a variety of conditional process models with multiple moderators for between-subjects designs. In theory all of these models could be recreated for the twoinstance repeated-measures design using the principles described in this dissertation. Just like multiple mediators, the primary difficulty would be implementation.

I've mentioned throughout this dissertation the issue of what to do when there are more than two-instances. This is even a fairly new topic in the between subjects literature. Hayes and Preacher (2014) describe how to conduct between-subjects mediation analysis when the independent variable is multicategorical. Judd et al. (2001) discuss some extension to multicategorical variables using orthogonal contrasts rather than difference scores in repeated-measures designs, but this has not yet been generalized to the path-analytic approach to mediation, moderation, or conditional process 
analysis. If the focal predictor is time and there are more than 2 measurements, there are a variety of structural equation modeling approaches that can examine longitudinal change, including latent growth curve modeling. Additionally, multilevel modeling could be used to fit these types of models easily. As was mentioned in the Limitations section, extensions to multicategorical independent variables are possible with the regression based approach, but these extensions seem much easier in other analytical approaches.

As discussed in Section 5.3, there are a variety of structural equation modeling methods which have been used to describe mediation processes with repeated measures data. However, these methods have yet to be generalized to conditional process models. Structural equation models have some advantages over regression models in that they can include measurement models and can be used to estimate covariance between parameters estimated in different outcome models. Additionally, structural equation modeling approaches can handle longitudinal change in a cleaner way than regression models. Generalizing latent difference score, latent growth curve, and cross-lag panel models to conditional process models would be advantageous as researchers could utilize the benefits of structural equation models and conditional process models for repeated-measures data all at the same time.

An additional future direction involves the integration of moderated mediation and equivalence testing. Researchers interested in establishing that an indirect effect is robust to some manipulation, or constant across individuals, would need to use a combination of equivalence testing and conditional process analysis. The third study in Chapter 4 is a good example of this. It may not be enough to say that the indirect effect does not significantly differ across order of presentation. This could be 
a Type II error, the probability of which is not controlled in traditional hypothesis testing approaches. Researchers interested in detecting order effects may want to make stronger claims that there is "no difference" or "minimal difference" across order, which would require an equivalence testing approach. Another example is in Dohle and Montoya (2017) where they tested if the indirect effect of drug name on dosage was moderated by type of drug. They found that the indirect effect was not significantly moderated. However, it would be useful to evaluate equivalence such that we could test if the indirect effect is the same across the two types of drugs. These issues have not previously been discussed together, and could add to the potential use of moderated mediation analysis.

\subsection{Summary}

This dissertation aimed to describe how researchers can conduct conditional process analysis in two-instance repeated-measures designs. I provided the basic vocabulary of mediation by describing it's implementation in between-subjects designs, an area many researchers may be more familiar with. I described recent developments in mediation and moderation for two-instance repeated measures designs. I then described how to combine mediation and moderation approaches to estimate conditional process models. Additionally, I defined a variety of statistics of interest: the index of moderated mediation and conditional indirect effects. These statistics can be used to make inference about the population and effects specifically of interest in moderated mediation hypotheses. Next, I provided three concrete examples of implementing these analyses in psychological data. These examples showed the variety of designs which these models can be used in and the variety of questions that can be answered 
using this analysis technique. It is important to note that the methods described in this dissertation are not the only methods available for analyzing data from this design. I described some alternative regression methods, multilevel models, and structural equation models that could be used for estimating conditional indirect effects in the two-instance repeated measures design. Finally, I discussed the limitation of the analysis technique and the two-instance repeated-measures design. I believe that researchers may find multilevel models easier to expand to complex designs. However, researchers only familiar with regression may find the difference score method proposed here an excellent bridge between between-subjects analytical methods and multilevel modeling. There is quite a bit more work to be done, as is clear by the Future Directions section. I hope this dissertation will facilitate the expansion of this model and increase discussion about the connections between analytical approaches for between-subjects and repeated-measures designs. 


\section{Appendix A: Mplus code for Lasselin et al. (2016) analysis}

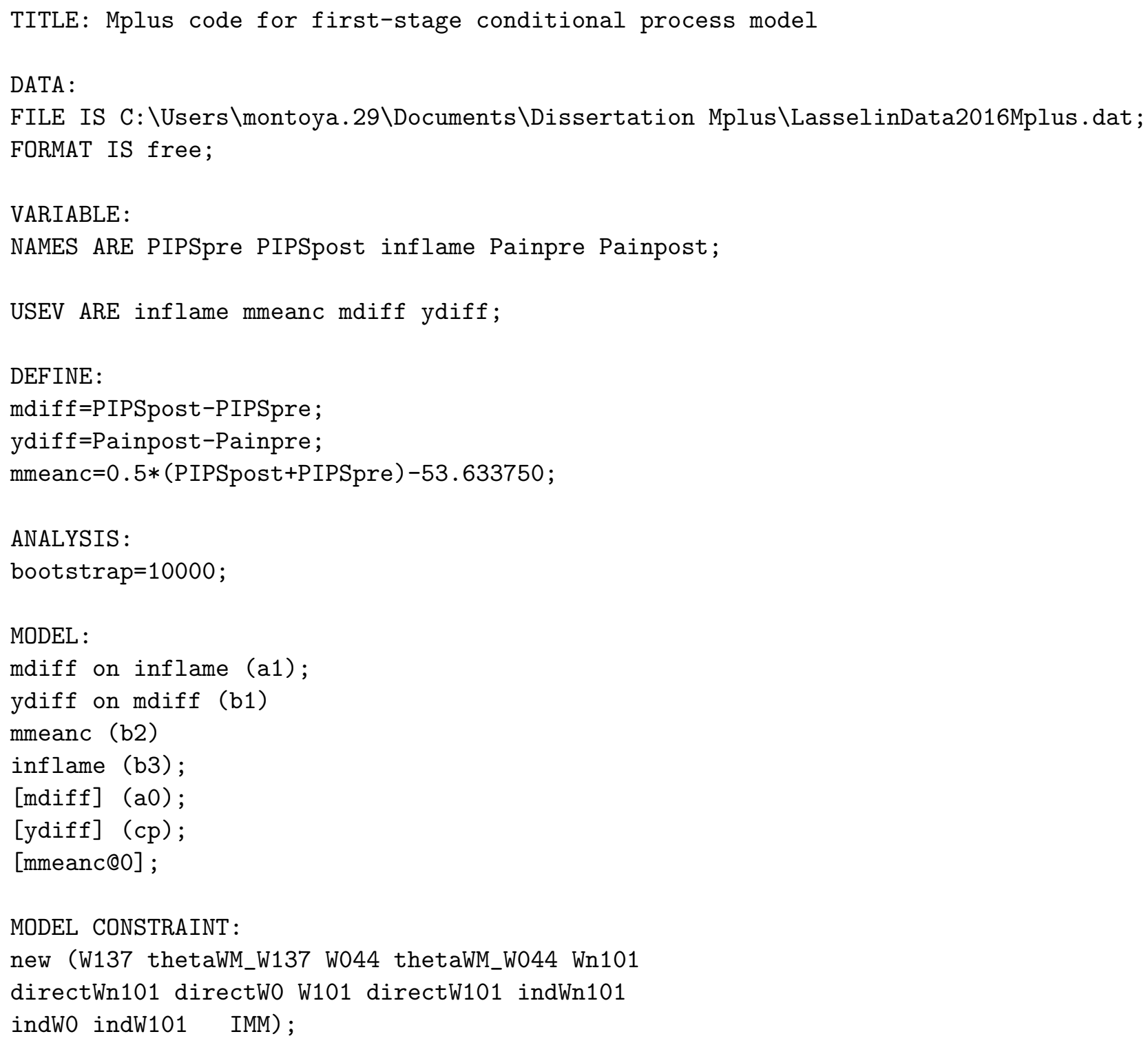




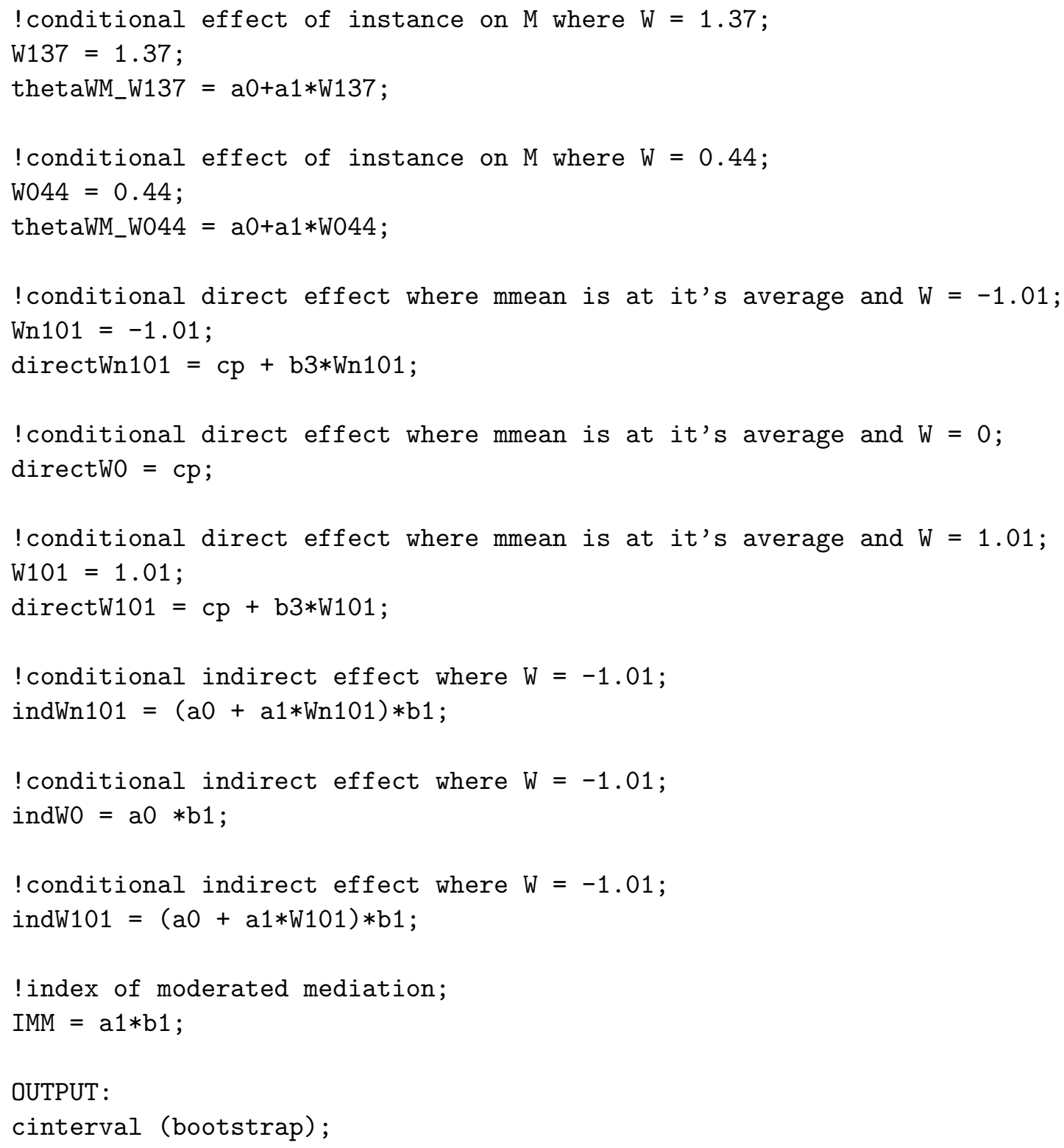




\section{Appendix B: Mplus code for Bell et al. (2017) analysis}

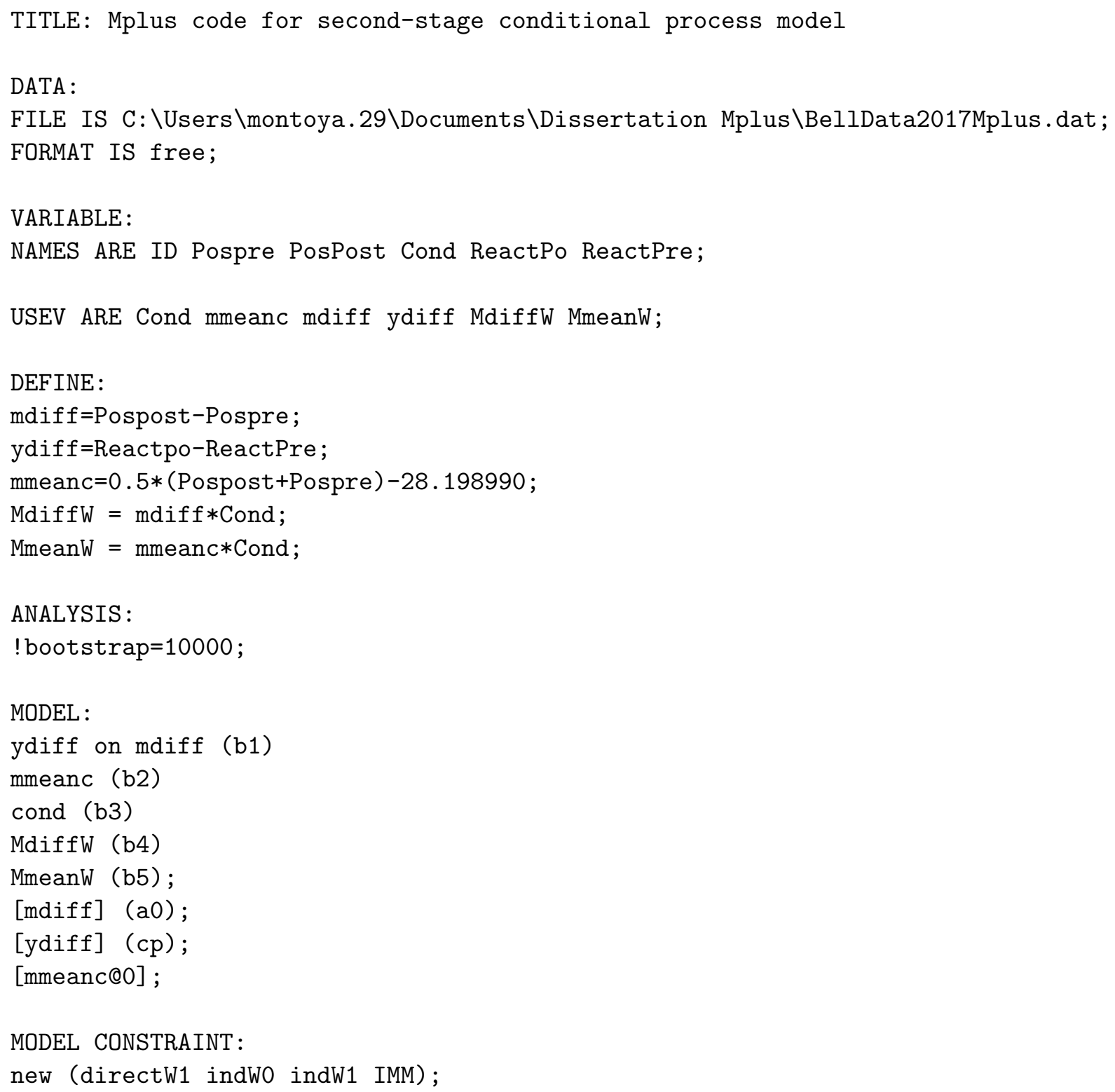


!conditional direct effect where mmean is at it's average and $W=1$;

directW1 $=c p+b 3$;

! conditional indirect effect where $\mathrm{W}=0$;

indW0 $=\mathrm{a} 0 * \mathrm{~b} 1$;

!conditional indirect effect where $W=0$;

indW1 $=\mathrm{a} 0 *(\mathrm{~b} 1+\mathrm{b} 4)$;

!index of moderated mediation;

$\mathrm{IMM}=\mathrm{a} 0 * \mathrm{~b} 4$;

OUTPUT :

!cinterval (bootstrap); 


\section{Appendix C: Mplus code for Montoya et al. (2013) analysis}

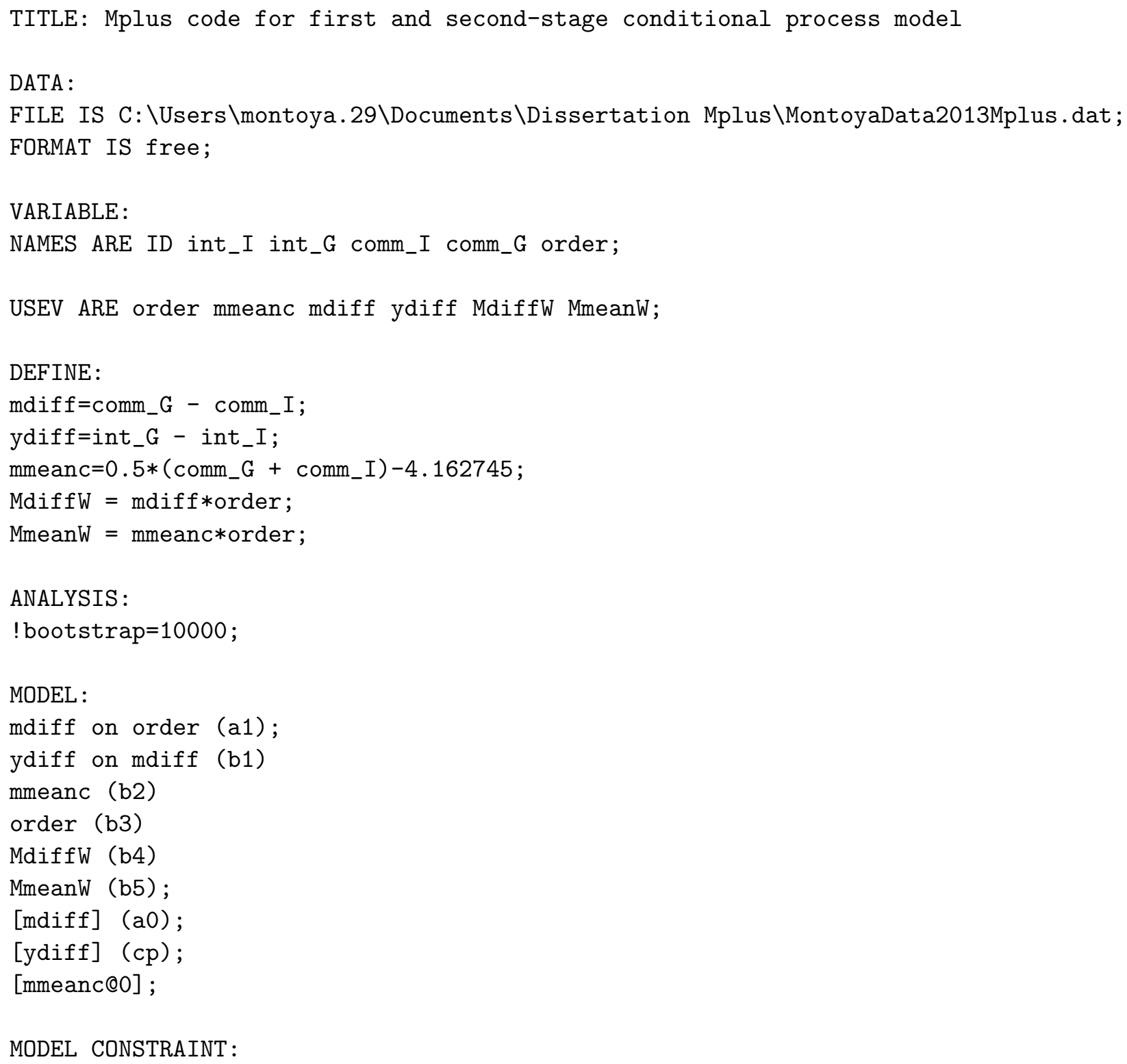




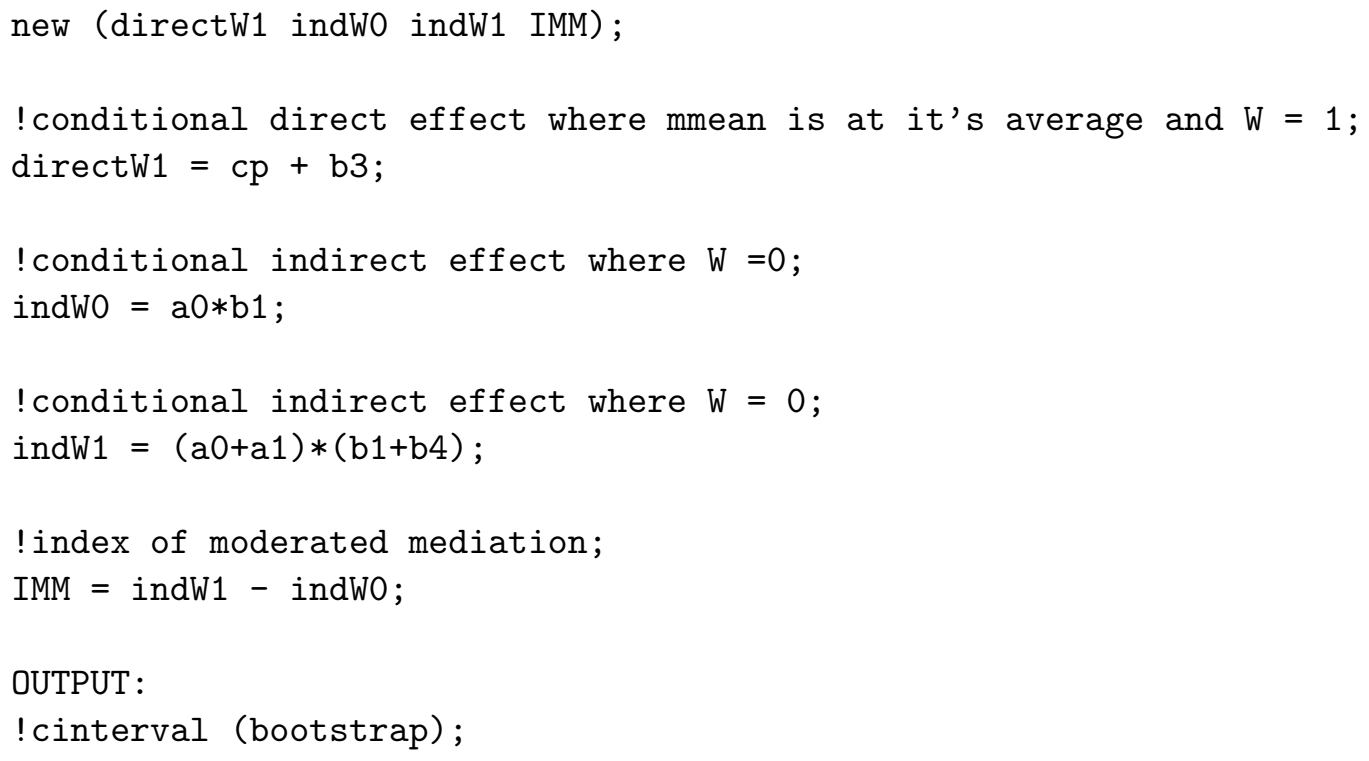




\section{References}

Baron, R. M., \& Kenny, D. A. (1986). The moderator-mediator variable distinction in social psychological research: Conceptual, strategic, and statistical considerations. Journal of Personality and Social Psychology, 51(6), 1173 - 1182.

Bauer, D. J., \& Curran, P. J. (2005). Probing interactions in fixed and multilevel regression: Inferential and graphical techniques. Multivariate Behavioral Research, 40(3), 373-400.

Bauer, D. J., Preacher, K. J., \& Gil, K. M. (2006). Conceptualizing and testing random indirect effects and moderated mediation in multilevel models: New procedures and recommendation. Psychological Methods, 11, 142 - 163.

Bell, Z., Shader, T., Webster-Stratton, C., Reid, M. J., \& Beauchaine, T. P. (2017). Improvements in negative parenting mediate changes in childrens autonomic responding following a preschool intervention for ADHD. Clinical Psychological Science, 1 - 11.

Biesanz, J. C., Falk, C. F., \& Savalei, V. (2010). Assessing mediational models: Testing and interval estimation for indirect effects. Multivariate Behavioral Research, 45, $661-701$.

Bonate, P. L. (2000). Analysis of pretest-posttest designs. New York: Chaptman \& Hall / CRC.

Breen, R., Karlson, K. B., \& Holm, A. (2013). Total, direct, and indirect effects in logic and probit models. Sociological Methods \& Research, 42(2), $164-191$.

Cheong, J. (2011). Investigation of mediational processes using latent growth curve modeling. Structural Equation Modeling, 18, 195 - 211.

Cheong, J., MacKinnon, D. P., \& Khoo, S. T. (2003). Investigation of mediational processes using latent growth curve modeling. Structural Equation Modeling, $18,195-211$.

Cheryan, S., Meltzoff, A. N., \& Kim, S. (2011). Classrooms matter: The design of virtual classrooms influences gender disparities in computer science classes. Computer \& Education, 57, 1825 - 1835.

Cohen, J., Cohen, P., West, S. G., \& Aiken, L. S. (2003). Applied multiple regression/correlation analysis for the behavioral sciences (3rd ed.). Mahwah, NJ: Erlbaum.

Cole, D. A., \& Maxwell, S. E. (2003). Testing mediational models with longitudinal data: Questions and tips in the use of structural equation modeling. Journal of Abnormal Psychology, 112, 558 - 577. 
Collins, L. M., Graham, J. J., \& Flaherty, B. P. (2010). An alternative framework for defining mediation. Multivariate Behavioral Research, 33(2), 295 - 312.

Cronbach, L. J., \& Furby, L. (1970). How we should measure "change" — or should we? Psychological Bulletin, 74(1), $68-80$.

Davis, M. (1985). The logic of causal order. In J. L. Sullivan \& R. G. Niemi (Eds.), Sage university paper series on quantitative applications in the social sciences. Beverly Hills: CA: Sage Publications.

Dohle, S., \& Montoya, A. K. (2017). The dark side of fluency: Fluent names increase drug dosing. Journal of Experimental Psychology: Applied, 23(3), 231 - 239.

Edwards, J. R., \& Lambert, L. S. (2007). Methods for integrating moderation and mediation: A general analytical framework using moderated path analysis. Psychological Methods, 12(1), $1-22$.

Efron, B., \& Tibshirani, R. J. (1993). An introduction to the bootstrap. Boca Raton, FL: Chapman \& Hall.

Fairchild, A. J., \& MacKinnon, D. P. (2009). A general model for testing mediation and moderation effects. Prevention Science, 10(2), 87 - 99.

Frazier, P. A., Tiz, A. P., \& Barron, K. E. (2004). Testing moderator and mediator effects in counseling psychology research. Journal of Counseling Psychology, $51(1), 115-134$.

Fritz, M. S., \& MacKinnon, D. P. (2007). Required sample size to detect the mediated effect. Psychological Science, $18(3), 233-239$.

Hayes, A. F. (2009). Beyond Baron and Kenny: Statistical mediation analysis in the new millennium. Communication Monographs, 76 (4), 408 - 420.

Hayes, A. F. (2013). Introduction to mediation, moderation, and conditional process analysis. New York, NY: Guilford Press.

Hayes, A. F. (2015). An index and test of linear moderated mediation. Multivariabe Behavioral Research, 50(1), $1-22$.

Hayes, A. F. (2018a). Introduction to mediation, moderation, and conditional process analysis (2nd ed.). New York, NY: Guilford Press.

Hayes, A. F. (2018b). Partial, conditional, and moderated moderated mediation: Quantification, inference, and interpretation. Communication Monographs, 85, $4-40$.

Hayes, A. F., \& Matthes, J. (2009). Computational procedures for probing interactions in OLS and logistic regression: SPSS and SAS implementations. Behavior Research Methods, 41(3), 924-936.

Hayes, A. F., Montoya, A. K., \& Rockwood, N. J. (2017). The analysis of mechanisms and their contingencies: PROCESS versus structural equation modeling. Australasian Marketing Journal, 25, 76 - 81.

Hayes, A. F., Montoya, A. K., \& Rockwood, N. J. (in prep). Mediation in pre-post designs. (Invited Submission)

Hayes, A. F., \& Preacher, K. J. (2014). Statistical mediation analysis with a multicategorical independent variable. British Journal of Mathematical and Statistical Psychology, 67, $451-470$. 
Hayes, A. F., \& Scharkow, M. (2013). The relative trustworthiness of inferential tests of the indirect effect in statistical mediation analysis: Does method really matter? Psychological Science, 24, 1918 - 1927.

Jaccard, J., \& Turrisi, R. (2003). Interaction effects in multiple regression (2nd ed.). Thousand Oaks, CA: Sage Publications.

James, L. R., \& Brett, J. M. (1984). Mediators, moderators, and tests for mediation. Journal of Applied Psychology, 69(2), 307 - 321.

Johnson, P. O., \& Fay, L. F. (1950). The Johnson-Neyman technique, its theory and application. Psychometrika, 15(4), 349-367.

Johnson, P. O., \& Neyman, J. (1936). Tests of certain linear hypotheses and their application to some educational problems. Statistical Research Memoirs, 1, 57-93.

Joslyn, S. L., \& LeClerc, J. E. (2016). Climate projections and uncertainty communication. Topics in Cognitive Science, 8, 222-241.

Judd, C. M., Kenny, D. A., \& McClelland, G. H. (2001). Estimating and testing mediation and moderation in within-subjects designs. Psychological Methods, $6(2), 115-134$.

Judd, C. M., McClelland, G. H., \& Smith, E. R. (1996). Testing treatment by covariate interactions when treatment varies within subjects. Psychological Methods, $1,366-378$.

Kenny, D. A. (1979). Correlation and causality. New York: Wiley.

Kenny, D. A., \& Judd, C. M. (2014). Power anomalies in testing mediation. Psychological Science, 25(2), $334-339$.

Kenny, D. A., Kashy, D. A., \& Bolger, N. (1998). Data analysis in social psychology. In D. T. Gilbert, S. T. Fiske, \& G. Lindzey (Eds.), The handbook of social psychology (pp. 233-265). Boston: McGraw-Hill.

Kenny, D. A., Korchmaros, J. D., \& Bolger, N. (2003). Lover-level mediation in multilevel models. Psychological Methods, 8(2), 115 - 128.

Krull, J. L., \& MacKinnon, D. P. (2001). Multilevel modeling of individual and gorup level mediated effects. Multivariate Behavioral Research, 36, 249 - 277.

Lasselin, J., Kemani, M. K., Kanstrup, M., Olsson, G. L., Axelsson, J., Andreasson, A., ... Wicksell, R. K. (2016). Low-grade inflammation may moderate the effect of behaviora ltreatment for chronic pain in adults. Journal of Behavioral Medicine, 39, 916 - 924.

Lord, F. E. (1963). Elementary models for measuring change. In C. W. Harris (Ed.), Problems in measuring change. Madison, Wisconsin: University of Wisconsin Press.

MacKinnon, D. P. (2008). Introduction to statistical mediation analysis. New York: Lawrence Erlbaum Associates.

MacKinnon, D. P., Lockwood, C. M., Hoffman, J. M., West, S. G., \& Sheets, V. (2002). A comparison of methods to test mediation and other intervening variable effects. Psychological Methods, 7, 83- 104.

MacKinnon, D. P., Lockwood, C. M., \& Williams, J. (2004). Confidence limits 
for the indirect effect: Distribution of the product and resampling methods. Multivariate Behavioral Research, 39, 99 - 128.

Manikandan, S., Nillni, Y. I., Zvolensky, M. J., Rohan, K. J., Carkeek, K. R., \& Leyro, T. M. (2016). The role of emotion regulation in the experience of menstrualsymptoms and perceived control over anxiety-related eventsacross the menstrual cycle. Archives of Women's Mental Health, 19(6), 1109 - 1117.

Maxwell, S. E., \& Cole, D. A. (2007). Bias in cross-sectional analyses of longitudinal mediation. Psychological Methods, 12, 23 - 44.

Maxwell, S. E., Cole, D. A., \& Mitchell, M. A. (2011). Bias in cross-sectional analyses of longitudinal mediation: partial and complete mediation under an autoregressive model. Psychological Methods, 12, 23 - 44.

McCabe, C. J., Kim, D. S., \& King, K. M. (2018). Improving present practices in the visual display of interactions. Advances in Methods and Practices in Psychological Science.

Mitchell, M. A., \& Maxwell, S. E. (2013). A comparison of the cross-sectional and sequential designs when assessing longitudinal mediation. Multivariate Behavioral Research, 48, $301-339$.

Montoya, A. K. (in press). Moderation analysis in two-instance repeated measures designs: Probing methods and multiple moderator models. Behavior Reasearch Methods.

Montoya, A. K., \& Hayes, A. F. (2017). Two-condition within-participant statistical mediation analysis: A path-analytic framework. Psychological Methods, 22, 6 -27 .

Montoya, A. K., Master, A. M., \& Cheryan, S. (2013). Increasing interest in computer science by adding group work: A goal congruity approach. (unpublished thesis)

Muller, D., Judd, C. M., \& Yzerbyt, V. Y. (2005). When moderation is mediated and mediation is moderated. Journal of Personality and Social Psychology, 89, $852-863$.

Powell, S. R., Cirino, P. T., \& Malone, A. S. (2017). Child-level predictors of responsiveness to evidence-based mathematics intervention. Exceptional Children, $83(4), 359-377$.

Preacher, K. J. (2015). Advances in mediation analysis: A survey and synthesis of new developments. Annual Review of Psychology, 66, 825-852.

Preacher, K. J., Curran, P. J., \& Bauer, D. J. (2006). Computational tools for probing interactions in multiple linear regression, multilevel modeling, and latent curve analysis. Journal of Educational \& Behavioral Statistics, 31(3), 437 - 488.

Preacher, K. J., \& Hayes, A. F. (2004). SPSS and SAS procedures for estimating indirect effects in simple mediation models. Behavior Research Methods, 36, $717-731$.

Preacher, K. J., Rucker, D. D., \& Hayes, A. F. (2007). Addressing moderated mediation hypotheses: Theory, methods, and prescriptions. Multivariate Behavioral Research, 42(1), $185-227$. 
Preacher, K. J., \& Selig, J. P. (2012). Advantages of Monte Carlo confidence intervals for indirect effects. Communication Methods and Measures, 6(2), 77 - 98.

Robinson, E. A., \& Eyberg, S. M. (1981). The dyadic parent-child interaction coding system: Standardization and validation. Journal of Consulting and Clinical Psychology, 49(2), $245-250$.

Rockwood, N. J. (2017). Advancing the formulation and testing of multilevel mediation and moderated mediation models (Unpublished master's thesis). The Ohio State University.

Rogosa, D. (1995). Myths and methods: "Myths about longitudinal research" plus supplemental questions. In J. M. Gottman (Ed.), The analysis of change. Mahwah, NJ: Lawrence Erlbaum Associates.

Roth, D. L., \& MacKinnon, D. P. (2012). Mediation analysis with longitudinal data. In J. T. Newsom, R. N. Jones, \& S. M. Hofer (Eds.), Longitudinal data analysis: A practical guide for researchers in aging, health, and social sciences (pp. 181 - 216). New York: Routledge.

Rucker, D. D., Preacher, K. J., \& Tormala, Z. L. (2011). Mediation analysis in social psychology: Current practice and new recommendations. Personality and Social Psychology Compass, 5/6, $359-371$.

Selig, J. P., \& Preacher, K. J. (2009). Mediation models for longitudinal data in developmental research. Research in Human Development, 6(2-3), $144-164$.

Shrout, P. E., \& Bolger, N. (2002). Mediation in experimental and nonexperimental studies: New procedures and recommendations. Psychological Methods, 7, 422 -445 .

Sobel, M. E. (1982). Asymptotic confidence intervals for indirect effects in structural equation models. Sociological Methodology, 13, $290-312$.

Spiller, S. A., Fitzimons, G. J., Lynch Jr., J. G., \& McClelland, G. H. (2013). Spotlights, floodlights, and the magic number zero: Simple effects tests in moderated regression. Journal of Marketing Research, 50, 277 - 288.

Stone, C. A., \& Sobel, M. E. (1990). The robustness of estimates of total indirect effects in covariance structure models estimated by maximum likelihood. Psychometrika, 55(2), $337-352$.

Thomas, D. R., \& Zumbo, B. D. (2012). Difference scores from the point of view of reliability and repeated-measures anova: In defense of difference scores for data analysis. Educational and Psychological Measurement, 72(1), 37 - 43.

Tong, J., Feiler, D., \& Ivantsova, A. (2017). Good choice, bad judgement: How choice under uncertainty generates overoptimism. Psychological Science, 29, $254-265$.

Twisk, J., \& Proper, K. (2004). Evaluation of the results of a randomized controlled trial:How to define change between baseline and followup. Journal of Clinical Epidemiology, 57, $223-228$.

Valente, M. J., \& MacKinnon, D. P. (2017). Comparing models of change to estimate the mediated effect in the pretest-posttest control group design. Structural Equation Modeling: A Multidisciplinary Journal, 23, 428 - 450. 
VanderWeele, T. J., Valeri, L., \& Ogburn, E. L. (2012). The role of measurement error and misclassification in mediation analysis. Epidemiology, 23(4), 561 564.

von Soest, T., \& Hagtvet, K. A. (2011). Mediation analysis in a latent growth curve modeling framework. Structural Equation Modeling, 18(2), 289 - 314.

Wicksell, R. K., Renofalt, J., Olsson, G. L., Bond, F. W., \& Melin, L. (2008). Avoidance and cognitive fusion central components in painrelated disability? development and preliminary validationof the psychological inflexibility in pain scale (pips). European Journal of Pain, 12, $491 \mathrm{f}-500$.

Williams, J., \& MacKinnon, D. P. (2008). Resampling and distribution of the product methods for testing indirect effects in complex models. Structural Equation Modeling, 15, $23-51$.

Winterich, K. P., Mittal, V., \& Ross, W. T. (2009). Donation behavior toward in-groups and out-groups: The role of gender and moral identity. Journal of Consumer Research, 36, 199 - 214.

World Health Organization. (2018). BMI classification. http://apps.who.int/bmi/index.jsp?introPage=intro.html. (Accessed: 2018-05-02)

Wu, W., Selig, J. P., \& Little, T. D. (2013). Longitudinal data analysis. In T. D. Little (Ed.), Handbook of quantitative methods. Oxford.

Zhang, Z., Zyphur, M. J., \& Preacher, K. J. (2009). Testing multilevel mediation using hierarchical linear models: Problems and solutions. Organizational Research Methods, 12(4), $695-719$.

Zumbo, B. D. (1999). The simple difference score as an inherently poor measure of change: Some reality, some mythology. In B. Thompson (Ed.), Advances in social science methodology, 5. Greenwich, CT: JAI Press. 